\title{
Exploring the geometry of supersymmetric double field theory
}

\section{Daniel Butter}

George P. and Cynthia W. Mitchell Institute for Fundamental Physics and Astronomy, Texas A $6 M$ University, College Station, TX 77843-4242, U.S.A.

E-mail: dbutter@tamu.edu

ABSTRACT: The geometry of $\mathcal{N}=1$ supersymmetric double field theory is revisited in superspace. In order to maintain the constraints on the torsion tensor, the local tangent space group of $\mathrm{O}(D) \times \mathrm{O}(D)$ must be expanded to include a tower of higher dimension generators. These include a generator in the irreducible hook representation of the Lorentz group, which gauges the shift symmetry (or ambiguity) of the spin connection. This gauging is possible even in the purely bosonic theory, where it leads to a Lorentz curvature whose only non-vanishing pieces are the physical ones: the generalized Einstein tensor and the generalized scalar curvature. A relation to the super-Maxwell $\infty$ algebra is proposed. The superspace Bianchi identities are solved up through dimension two, and the component supersymmetry transformations and equations of motion are explicitly (re)derived.

Keywords: Superspaces, String Duality, Supergravity Models, Supersymmetry and Duality

ArXIV EPrint: 2101.10328 


\section{Contents}

1 Introduction 1

2 Bosonic geometry of double field theory 3

2.1 Review of bosonic DFT geometry 3

2.2 Extending the geometry 8

3 Double field theory in superspace 12

3.1 Elements of component supersymmetric DFT 12

3.2 Siegel's superspace DFT 12

$\begin{array}{lll}3.3 & \text { Determining the tangent space } & 14\end{array}$

4 Superspace geometry and its constraints $\quad 17$

$\begin{array}{ll}\text { 4.1 Generalized fluxes and their constraints } & 17\end{array}$

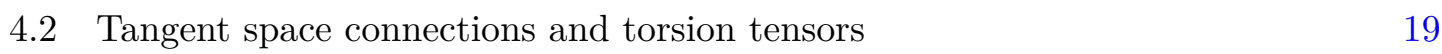

4.3 A detour on tangent space symmetries and an extended algebra 26

4.4 New connections, transformations, and curvatures 30

4.5 Solution of the Bianchi identities through dimension 2

5 Derivation of component supersymmetric DFT

5.1 Conventional $\mathcal{N}=1$ superspace: a review and a redo 37

5.2 Level decomposition of the doubled supervielbein 40

5.3 Generalized diffeomorphisms 43

5.4 Supersymmetry transformations 44

5.5 The component spin connection 46

5.6 Supercovariant gravitino curvatures and fermionic equations of motion 48

5.7 Supercovariant Lorentz curvatures and bosonic equations of motion 50

5.8 Comparing with component DFT and $\mathcal{N}=1$ supergravity $\quad 52$

6 Open questions $\quad 54$

A Notations and conventions $\quad 56$

$\begin{array}{lll}\text { A.1 } & \operatorname{Spin}(9,1) \text { conventions } & 56\end{array}$

$\begin{array}{lll}\text { A.2 } & \mathrm{OSp}(p, q \mid 2 s) \text { conventions } & 57\end{array}$

$\begin{array}{ll}\text { B Poláček-Siegel formulation of DFT with connections } & 57\end{array}$

C Construction of the extended super-Maxwell $\infty$ algebra $\quad 64$

$\begin{array}{lll}\text { C.1 The super-Maxwell } \infty \text { algebra } & 64\end{array}$

$\begin{array}{lll}\text { C.2 Local Lie superalgebra construction } & 69\end{array}$ 


\section{Introduction}

Double field theory (DFT) is a formulation of the massless sector of string theory that makes $T$-duality manifest [1-6]. It describes the low energy sector of bosonic string theory (or the bosonic NS-NS sector of supersymmetric string theory) and unifies the metric and two-form together into a generalized metric, which transforms under generalized diffeomorphisms governed by the group $\mathrm{O}(D, D)$. In order to incorporate fermions and supersymmetry, one requires a frame formulation $[1,2,7-9]$, and in this approach the generalized vielbein is an element of $\mathrm{O}(D, D)$ with a doubled tangent space group $\mathrm{O}(D-1,1) \times \mathrm{O}(D-1,1)$. For further references and discussions of important applications of DFT, we refer the reader to the reviews $[10,11]$.

The topic of this paper is the structure of supersymmetric double field theory, and specifically how to deduce it from a doubled supergeometry. At the component level, the structure of $\mathcal{N}=1$ double field theory was identified to second order in fermions in [12] and to all orders in [13] (see also [14]). Remarkably, the prescient early papers by Siegel $[1,2]$ already contained a superspace description, from which one should be able to deduce supersymmetry transformations and equations of motion. In principle this ought to be a straightforward task, and the original motivation of this paper was simply to analyze this example as a warm-up to the more interesting type II and exceptional cases. ${ }^{1}$

Surprisingly, this is not as straightforward as it might seem. Aside from the technical aspects of reducing a superspace formulation to a component one - similar to more conventional superspaces, albeit a bit more technically involved when working with generalized super-diffeomorphisms - one encounters a roadblock absent in conventional superspaces or even in bosonic DFT. In order for the supervielbein to be an unconstrained element of a supergroup - the relevant one here being the orthosymplectic group $\operatorname{OSp}(D, D \mid 2 s)$ with $D=10$ and $s=16$ - the local tangent space symmetry must be extended beyond the doubled Lorentz group $\mathrm{O}(D-1,1) \times \mathrm{O}(D-1,1)$ familiar in the bosonic theory. This is because the supervielbein carries a large number of degrees of freedom, and only some of them can be eliminated using the usual Wess-Zumino gauge-fixing conditions. To guarantee the remaining components drop out, an enhanced local gauge symmetry is necessary. This was implicit already in [2], where it was used to eliminate these additional fields almost immediately. Our new observation is that consistency with the supersymmetry algebra implies that this is just the first extension of a (possibly infinite) tower of local gauge symmetries. Only the lowest few levels of these symmetries act on the supervielbein via a tangent space rotation.

This extension turns out to not purely be an artifact of superspace. It casts a shadow on the purely bosonic theory as well. It is a fact of life that in the frame formulation of DFT, the spin connection is not fully determined by the vanishing torsion conditions. This can

\footnotetext{
${ }^{1} \mathcal{N}=2$ supersymmetric double field theory (that is, for type II strings) has been constructed at the component level [15], extending the bosonic construction of $[16,17] . \mathcal{N}=2$ superspace formulations have been given in [18-20] (see also [21, 22]). Supersymmetric exceptional field theories have been built for $E_{6}$, $E_{7}$, and $E_{8}$ [23-25] (with partial results for $E_{11}[26]$ ), but have been discussed in superspace only for the $E_{7}$ case [27]. Note that an off-shell formulation of $11 \mathrm{D}$ superspace exists involving pure spinors [28-30], and it would be interesting to explore whether a duality-covariant generalization is possible.
} 
be restated in the following way: the purely left-handed and right-handed components of the spin connection are ambiguous under shifts in the irreducible hook representations, i.e.

$$
\delta \omega_{a b c}=\Lambda_{a \mid b c}, \quad \delta \omega_{\overline{a b c}}=\Lambda_{\bar{a} \mid \overline{b c}},
$$

where $\Lambda_{[a \mid b c]}=\Lambda_{\mid b c}^{b}=0$ and similarly for $\Lambda_{\bar{a} \mid \overline{b c}}$. Usually this is handled by requiring that these representations of $\omega$ drop out of all physical quantities (actions, equations of motion, etc.). However, in principle, there is no reason why one cannot demand that (1.1) be an honest local gauge invariance, which we impose to eliminate unphysical degrees of freedom. After all, the doubled Lorentz group is introduced to eliminate the unphysical degrees of freedom in the doubled vielbein. What we discover in superspace is this new local symmetry is actually required by closure of the extended tangent space algebra. In other words, it transforms a "bug" of DFT into a "feature."

This new local symmetry naturally implies others. This is because its gauging necessitates the introduction of new gauge fields $h_{\hat{a}, b \mid c d}$ and $h_{\hat{a}, \bar{b} \mid \overline{c d}}$. In order to not introduce new degrees of freedom, these gauge fields should be determined in terms of the generalized vielbein in some way. What happens is that just as $\omega$ appears algebraically in the torsion tensor, $h$ appears algebraically in the Lorentz curvature. Imposing that the torsion tensor vanishes determines most of $\omega$, and similarly imposing that some components of the Lorentz curvature tensor vanish determines most of $h$. But because $h$ itself retains a residual shift symmetry analogous to (1.1), the process may continue, with further connections and curvatures introduced at ever higher dimensions. An important consequence of all of these additional symmetries is that the only non-vanishing components of the generalized curvature tensors (torsion, Lorentz curvature, etc.) that survive are the physical ones.

In attempting to make sense of this hierarchy, we have drawn inspiration from the work of Poláček and Siegel [31]. They showed how one could incorporate the spin connection into a larger megavielbein by extending the doubled spacetime so that the extended derivative $D_{\mathcal{M}}=\left(M_{\hat{a} \hat{b}}, \partial_{\hat{m}}, \widetilde{M}^{\hat{a} \hat{b}}\right)$ includes the $\mathrm{SO}(D-1,1) \times \mathrm{SO}(D-1,1)$ Lorentz generator $M_{\hat{a} \hat{b}}$, along with a composite dual generator $\widetilde{M}^{\hat{a} \hat{b}}$, required so that the extended metric $\eta_{\mathcal{M N}}$ is invertible. In gauging the shift symmetry (1.1), its descendants, and their superpartners, we find that the dual generators extending $\widetilde{M}^{\hat{a} \hat{b}}$ can be embedded in a version of the super-Maxwell $_{\infty}$ algebra (see recent discussions in $[32,33]$ ). It is unclear to us if this fully characterizes the super-algebra or if a quotient must be taken. We discuss some of these issues in the conclusion.

The paper is arranged as follows. We begin in section 2 with a discussion of the geometry of bosonic double field theory. This is largely for review and to fix notation, but we elaborate on the shift symmetry of the spin connection and how the introduction of the higher connection $h$ permits one to build a Lorentz curvature tensor with only physical components. In section 3 we extend the formalism to superspace. This is primarily a review of [2] but in modified language and formalism. Particular attention is paid to how the tangent space group is used to eliminate unphysical components of the vielbein. This is followed by section 4 , where we explicitly analyze the generalized torsion and curvature tensors up through dimension 2. It is here that we uncover the need for an extended 


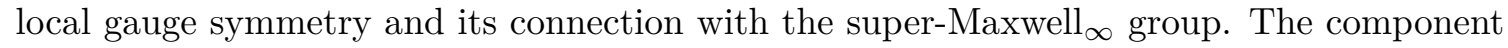
supersymmetry transformations and equations of motion are derived in section 5 and perfect agreement is found with $[12,13]$. As is typical for superspaces with maximal supersymmetry, the lower dimension $(\leq 1 / 2)$ torsion constraints effectively place the theory on-shell. ${ }^{2}$ Finally in section 6 , we discuss several open questions and possible extensions.

Several technical appendices are included. Appendix A summarizes the conventions we use. Appendix B reviews the Poláček-Siegel (PS) formalism [31] for embedding connections into a megavielbein. While we do not use such a megavielbein explicitly in the main body of the paper, the PS formalism does provide a useful explanation for the transformation rules of the connections and the construction of the curvature tensors when a generic group is gauged in DFT. Appendix C gives the construction of the super-Maxwell $\infty$ algebra and its extension, which appears to play a role in building the local gauge group of $\mathcal{N}=1$ DFT.

\section{Bosonic geometry of double field theory}

Let us begin with a discussion of bosonic double field theory. This is well-studied material over the last decade, so we will be relatively brief, highlighting only the geometry of covariant derivatives, connections, etc. that we will be extending to the supersymmetric case. The major difference with conventional formulations is that we include two new connection-like fields. The first is the Poláček-Siegel field that permits the construction of a generalized doubled Lorentz curvature [31]. The second is the new connection $h$ that transforms in the irreducible hook representation of the doubled Lorentz group. This new connection gauges shift symmetries in the spin connection. Together these fields are responsible for eliminating all but the physical components of the doubled Lorentz curvature.

\subsection{Review of bosonic DFT geometry}

The material here mostly follows that of $[2,7]$ with only superficial alterations.

We begin with a bosonic space with a doubled set of coordinates $x^{\hat{m}}, \hat{m}=1, \cdots, 2 D$. Typically these coordinates are denoted $x^{M}$ but we want to reserve the index $M$ for the supersymmetric case later on. The space is furnished with a constant invariant $\mathrm{O}(D, D)$ metric $\eta^{\hat{m} \hat{n}}$ (and inverse $\eta_{\hat{m} \hat{n}}$ ), with which we can raise (or lower) indices. A generalized diffeomorphism of a vector $W^{\hat{m}}$ with weight $w$ is given by

$$
\delta_{\xi} W^{\hat{m}}=\mathbb{L}_{\xi}^{(w)} W^{\hat{m}}:=\xi^{\hat{n}} \partial_{\hat{n}} W^{\hat{m}}-W^{\hat{n}}\left(\partial_{\hat{n}} \xi^{\hat{m}}-\partial^{\hat{m}} \xi_{\hat{n}}\right)+w \partial_{\hat{n}} \xi^{\hat{n}} W^{\hat{m}} .
$$

These are $\mathrm{O}(D, D) \times \mathbb{R}^{+}$generalized Lie derivatives. Requiring closure of the algebra implies the section condition,

$$
\eta^{\hat{m} \hat{n}} \partial_{\hat{m}} \otimes \partial_{\hat{n}}=0
$$

with the derivatives understood to act on either the same or different objects. By construction, the weight-zero tensor $\eta$ is invariant under generalized diffeomorphisms.

\footnotetext{
${ }^{2}$ The notable exception is off-shell pure spinor superspace [28-30], but the connection to conventional supergeometry is obscured there.
} 
There are two different coordinate systems one commonly encounters in DFT. The first is the standard coordinate system, with

$$
x^{\hat{m}}=\left(x^{\mathrm{m}}, \tilde{x}_{\mathrm{m}}\right), \quad \partial_{\hat{m}}=\left(\partial_{\mathrm{m}}, \tilde{\partial}^{\mathrm{m}}\right), \quad \eta^{\hat{m} \hat{n}}=\left(\begin{array}{cc}
0 & \delta^{\mathrm{m}} \mathrm{n} \\
\delta_{\mathrm{m}}{ }^{\mathrm{n}} & 0
\end{array}\right)
$$

where $\mathrm{m}=1, \cdots, D$. In toroidal compactifications of string theory, $x^{\mathrm{m}}$ corresponds to the center-of-mass coordinate of the string and $\tilde{x}_{\mathrm{m}}$ corresponds to the coordinate dual to the winding momenta. The second is the left/right-moving coordinate system, where the center-of-mass and winding mode coordinates are combined as $x^{m}=\frac{1}{\sqrt{2}}\left(x^{\mathrm{m}}+\eta^{\mathrm{mn}} \tilde{x}_{\mathrm{n}}\right)$ and $x^{\bar{m}}=\frac{1}{\sqrt{2}}\left(x^{\mathrm{m}}-\eta^{\mathrm{mn}} \tilde{x}_{\mathrm{n}}\right)$, where $\eta^{\mathrm{mn}}$ is the $\mathrm{SO}(D-1,1)$ metric. In this coordinate system,

$$
\eta^{\hat{m} \hat{n}}=\left(\begin{array}{cc}
\eta^{m n} & 0 \\
0 & -\eta^{\bar{m} \bar{n}}
\end{array}\right)
$$

where $\eta^{\bar{m} \bar{n}}=\eta^{m n}$. $^{3}$ Typically, one works in the standard coordinate system for the world indices but in the left/right-moving basis for tangent space indices.

The metric of DFT is a symmetric tensor $\mathcal{H}_{\hat{m} \hat{n}}$ subject to an invariance condition

$$
\mathcal{H}^{\hat{m} \hat{n}}:=\eta^{\hat{m} \hat{m}^{\prime}} \eta^{\hat{n} \hat{n}^{\prime}} \mathcal{H}_{\hat{m}^{\prime} \hat{n}^{\prime}}=\left(\mathcal{H}^{-1}\right)^{\hat{m} \hat{n}}
$$

The DFT metric may be decomposed in the standard coordinate basis as

$$
\mathcal{H}_{\hat{m} \hat{n}}=\left(\begin{array}{cc}
g_{\mathrm{mn}}-b_{\mathrm{mk}} g^{\mathrm{kl}} b_{\mathrm{ln}} & b_{\mathrm{mk}} g^{\mathrm{kn}} \\
-g^{\mathrm{mk}} b_{\mathrm{kn}} & g^{\mathrm{mn}}
\end{array}\right), \quad \quad \mathcal{H}^{\hat{m} \hat{n}}=\left(\begin{array}{cc}
g^{\mathrm{mn}} & -g^{\mathrm{mk}} b_{\mathrm{kn}} \\
b_{\mathrm{mk}} g^{\mathrm{kn}} & g_{\mathrm{mn}}-b_{\mathrm{mk}} g^{\mathrm{kl}} b_{\mathrm{ln}}
\end{array}\right) .
$$

In supersymmetric DFT, as in conventional supergravity, it is necessary to introduce a vielbein along with its associated tangent space. Here, the vielbein is a field $V_{\hat{m}}{ }^{\hat{a}}$ subject to two conditions:

$$
\mathcal{H}_{\hat{m} \hat{n}}=V_{\hat{m}}^{\hat{a}} V_{\hat{n}}^{\hat{b}} \mathcal{H}_{\hat{a} \hat{b}}, \quad \eta_{\hat{m} \hat{n}}=V_{\hat{m}}^{\hat{a}} V_{\hat{n}}^{\hat{b}} \eta_{\hat{a} \hat{b}},
$$

where $\mathcal{H}_{\hat{a} \hat{b}}$ and $\eta_{\hat{a} \hat{b}}$ are fixed constant tensors. The second condition implies that the vielbein is an $\mathrm{O}(D, D)$ element, with its inverse given by $V_{\hat{a}}^{\hat{m}}=\eta_{\hat{a} \hat{b}} \eta^{\hat{m} \hat{n}} V_{\hat{n}}^{\hat{b}}$. In the standard parametrization of the tangent space group, we choose

$$
\mathcal{H}_{\hat{a} \hat{b}}=\left(\begin{array}{cc}
\eta_{\mathrm{ab}} & 0 \\
0 & \eta^{\mathrm{ab}}
\end{array}\right), \quad \eta_{\hat{a} \hat{b}}=\left(\begin{array}{cc}
0 & \delta_{\mathrm{a}}^{\mathrm{b}} \\
\delta_{\mathrm{b}}^{\mathrm{a}} & 0
\end{array}\right),
$$

where $\eta_{\mathrm{ab}}$ corresponds to the standard $\mathrm{O}(D-1,1)$ metric. These tensors are invariant under local infinitesimal tangent space transformations with

$$
\lambda_{\hat{a}}^{\hat{b}}=\left(\begin{array}{cc}
\lambda_{\mathrm{a}}^{\mathrm{b}} & \tilde{\lambda}_{\mathrm{ab}} \\
\tilde{\lambda}^{\mathrm{ab}} & \lambda_{\mathrm{b}}^{\mathrm{a}}
\end{array}\right)
$$

\footnotetext{
${ }^{3}$ This is potentially confusing notation since $(\eta)^{\bar{m} \bar{n}}=-\eta^{\bar{m} \bar{n}}$. We never employ the former explicitly.
} 
where $\lambda$ and $\tilde{\lambda}$ are antisymmetric and their indices are raised and lowered with $\eta_{\mathrm{ab}}$. Up to a local tangent space transformation, the vielbein can be chosen in an upper triangular gauge as

$$
V_{\hat{m}}^{\hat{a}}=\left(\begin{array}{cc}
e_{\mathrm{m}}{ }^{\mathrm{a}} & b_{\mathrm{mn}} e_{\mathrm{a}}^{\mathrm{n}} \\
0 & e_{\mathrm{a}}{ }^{\mathrm{m}}
\end{array}\right), \quad V_{\hat{a}}^{\hat{m}}=\left(\begin{array}{cc}
e_{\mathrm{a}}^{\mathrm{m}}-e_{\mathrm{a}}{ }^{\mathrm{n}} b_{\mathrm{nm}} \\
0 & e_{\mathrm{m}}{ }^{\mathrm{a}}
\end{array}\right),
$$

This is not the most convenient parametrization for either the string worldsheet or supersymmetry. Instead, one may introduce left-handed indices $a, b, \cdots$ and right-handed indices $\bar{a}, \bar{b}, \cdots$, on which the tangent space group acts separately, as $\mathrm{O}(D-1,1)_{L} \times \mathrm{O}(D-$ $1,1)_{R}$. In this approach,

$$
\eta_{\hat{a} \hat{b}}=\left(\begin{array}{cc}
\eta_{a b} & 0 \\
0 & -\eta_{\overline{a b}}
\end{array}\right), \quad \mathcal{H}_{\hat{a} \hat{b}}=\left(\begin{array}{cc}
\eta_{a b} & 0 \\
0 & \eta_{\overline{a b}}
\end{array}\right)
$$

where $\eta_{\overline{a b}}=\eta_{a b}$. We choose to raise and lower barred indices with $\eta_{\overline{a b}}$ rather than $(\eta)_{\overline{a b}}$, so this means one must be careful when decomposing a vector to specify whether the index is raised or lowered. In the left/right tangent basis, the vielbein is given by

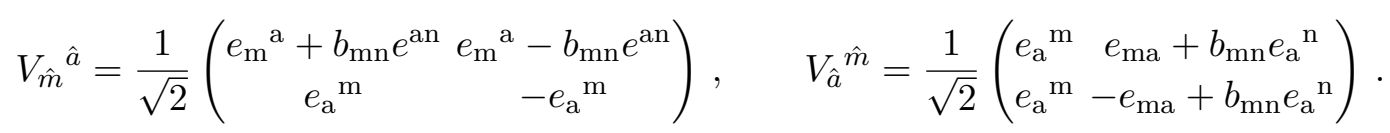

In either basis, the DFT vielbein transforms infinitesimally under generalized diffeomorphisms and $\mathrm{O}(D-1,1)_{L} \times \mathrm{O}(D-1,1)_{R}$ transformations as

$$
\delta V_{\hat{m}}^{\hat{a}}=\xi^{\hat{n}} \partial_{\hat{n}} V_{\hat{m}}^{\hat{a}}+V_{\hat{n}}^{\hat{a}}\left(\partial_{\hat{m}} \xi^{\hat{n}}-\partial^{\hat{n}} \xi_{\hat{m}}\right)-V_{\hat{m}}^{\hat{b}} \lambda_{\hat{b}}^{\hat{a}} .
$$

In addition to the DFT vielbein, we also need the dilaton. We choose to treat the dilaton as a weight-1 scalar density, $\phi=e^{-2 d}$, where $d$ is the usual DFT dilaton. $\phi$ transforms as

$$
\delta \phi=\xi^{\hat{m}} \partial_{\hat{m}} \phi+\partial_{\hat{m}} \xi^{\hat{m}} \phi=\partial_{\hat{m}}\left(\xi^{\hat{m}} \phi\right) .
$$

We trust this notation will not be too confusing. Later on, we will need to refer to the standard (non-density) dilaton of supergravity, which we denote $\varphi$. These are related by

$$
\phi=e^{-2 d}=\operatorname{det} e_{\mathrm{m}}^{\mathrm{a}} e^{-2 \varphi} .
$$

Together the dilaton and the vielbein can be combined into a weight- 1 vielbein, which is an element of $\mathrm{O}(D, D) \times \mathbb{R}^{+}$. However, it is more convenient (not to mention conventional) to keep them as distinct fields. The vielbein and the dilaton together can be used to construct two generalized fluxes $F_{\hat{a} \hat{b} \hat{c}}$ and $F_{\hat{a}}$. These are given by (note the unconventional sign choices)

$$
\begin{aligned}
\mathbb{L}_{V_{\hat{a}} V_{\hat{b}}^{\hat{m}}}=-F_{\hat{a} \hat{b}}^{\hat{c}} V_{\hat{c}}^{\hat{m}} & \Longrightarrow & F_{\hat{a} \hat{b} \hat{c}}=-3 D_{[\hat{a}} V_{\hat{b}}^{\hat{m}} V_{\hat{m} \hat{c}]}, \\
\mathbb{L}_{V_{\hat{a}}}^{(+1)} \phi=F_{\hat{a}} \phi & \Longrightarrow & F_{\hat{a}}=D_{\hat{a}} \log \phi+\partial_{\hat{m}} V_{\hat{a}}^{\hat{m}} .
\end{aligned}
$$


The flattened derivatives $D_{\hat{a}}:=V_{\hat{a}}^{\hat{m}} \partial_{\hat{m}}$ obey

$$
\left[D_{\hat{a}}, D_{\hat{b}}\right]=-F_{\hat{a} \hat{b}}^{\hat{c}} D_{\hat{c}}, \quad D^{\hat{a}} D_{\hat{a}}=-F^{\hat{a}} D_{\hat{a}} .
$$

The unconventional choice of sign for the flux $F_{\hat{a} \hat{b} \hat{c}}$ is to match our conventions for the torsion tensor later on in superspace.

These flux tensors are covariant under generalized diffeomorphisms, but transform inhomogeneously under Lorentz transformations. This latter issue can be rectified by introducing by hand a spin connection $\omega_{\hat{m} \hat{a}}^{\hat{b}}$ transforming as

$$
\delta \omega_{\hat{m} \hat{a}}^{\hat{b}}=\mathbb{L}_{\xi} \omega_{\hat{m} \hat{a}}^{\hat{b}}+\partial_{\hat{m}} \lambda_{\hat{a}}^{\hat{b}}+\lambda_{\hat{a}}{ }^{\hat{c}} \omega_{\hat{m} \hat{c}}^{\hat{d}}-\omega_{\hat{m} \hat{a}}{ }^{\hat{c}} \lambda_{\hat{c}}{ }^{\hat{d}} .
$$

Because of the sign in $\eta_{\hat{a} \hat{b}}$ in (2.11), one must be careful about how $\omega$ is defined. We take

$$
\omega_{\hat{m} \hat{a} \hat{b}}=\left(\omega_{\hat{m} a b}, \omega_{\hat{m} \overline{a b}}\right), \quad \omega_{\hat{m} \hat{a}}^{\hat{b}}=\left(\omega_{\hat{m} a^{b}},-\omega_{\hat{m} \bar{a}} \bar{b}^{\bar{b}}\right),
$$

and similarly for the Lorentz parameter $\lambda$.

Now the flux tensors may be modified to generalized torsion tensors $T_{\hat{a} \hat{b} \hat{c}}$ and $T_{\hat{a}}$ by replacing derivatives with covariant derivatives. In our conventions, $\mathcal{D}_{\hat{a}} V_{\hat{b}}=D_{\hat{a}} V_{\hat{b}}-\omega_{\hat{a} \hat{b}} \hat{c} V_{\hat{c}}$, so that

$$
\begin{aligned}
T_{\hat{a} \hat{b} \hat{c}} & :=-3 \mathcal{D}_{[\hat{a}} V_{\hat{b}}^{\hat{m}} V_{\hat{m} \hat{c}]}=F_{\hat{a} \hat{b} \hat{c}}+3 \omega_{[\hat{a} \hat{b} \hat{c}]}, \\
T_{\hat{a}} & :=\mathcal{D}_{\hat{a}} \log \phi+\mathcal{D}_{\hat{m}} V_{\hat{a}}^{\hat{m}}=F_{\hat{a}}+\omega^{\hat{b}} \hat{b} \hat{a}
\end{aligned}
$$

Setting to zero these torsions fixes the spin connection $\omega_{\hat{a} \hat{b} \hat{c}}$ up to the irreducible hook representations in the left and right Lorentz groups. In other words, the spin connection is defined only up to local transformations

$$
\delta \omega_{a b c}=\Lambda_{a \mid b c}, \quad \delta \omega_{\overline{a b c}}=\Lambda_{\bar{a} \mid \overline{b c}}
$$

where $\Lambda_{a \mid b c}$ obeys (and similarly for $\Lambda_{\bar{a} \mid \overline{b c}}$ )

$$
\Lambda_{a \mid b c}=-\Lambda_{a \mid c b}, \quad \Lambda_{[a \mid b c]}=0, \quad \Lambda_{\mid b c}^{b}=0 .
$$

There is no direct analogue to the Riemann tensor in DFT. A close runner-up is

$$
R(\omega)_{\hat{a} \hat{b} \hat{c} \hat{d}}:=2 D_{[\hat{a}} \omega_{\hat{b}] \hat{c} \hat{d}}-2 \omega_{[\hat{a} \mid \hat{c}} \hat{e} \omega_{\mid \hat{b}] \hat{e} \hat{d}}+F_{\hat{a} \hat{b}}{ }^{\hat{e}} \omega_{\hat{e} \hat{c} \hat{d}}+\frac{1}{2} \omega^{\hat{e}} \hat{a} \hat{b} \omega_{\hat{e} \hat{c} \hat{d}}
$$

which is covariant under diffeomorphisms but slightly non-covariant under Lorentz transformations. Its Lorentz non-covariance is

$$
\Delta_{\lambda} R(\omega)_{\hat{a} \hat{b} \hat{c} \hat{d}}=-\frac{1}{2} D^{\hat{e}} \lambda_{\hat{a} \hat{b}} \omega_{\hat{e} \hat{c} \hat{d}}+\frac{1}{2} D^{\hat{e}} \lambda_{\hat{c} \hat{d}} \omega_{\hat{e} \hat{a} \hat{b}}
$$

where $\Delta_{\lambda}$ denotes the difference between the full Lorentz transformation and the covariant part. This means that while $R(\omega)_{a \bar{b} c d}$ and $R(\omega)_{a \bar{b} \overline{c d}}$ are covariant, $R(\omega)_{a b c d}, R(\omega)_{a b \overline{c d}}$, 
$R(\omega)_{\overline{a b} c d}$, and $R(\omega)_{\overline{a b} \overline{c d}}$ are not. Nevertheless, because (2.25) is pairwise antisymmetric in $\hat{a} \hat{b}$ and $\hat{c} \hat{d}$, one may construct a symmetrized Riemann tensor

$$
\mathcal{R}_{\hat{a} \hat{b} \hat{c} \hat{d}}=\frac{1}{2} R(\omega)_{\hat{a} \hat{b} \hat{c} \hat{d}}+\frac{1}{2} R(\omega)_{\hat{c} \hat{d} \hat{a} \hat{b}}
$$

that is covariant under Lorentz transformations. This is the object that is usually considered the analogue of the doubled Riemann tensor.

Unfortunately, $\mathcal{R}_{\hat{a} \hat{b} \hat{c} \hat{d}}$ generally depends on undetermined parts of the spin connection. Put another way, it is not a tensor under the shift symmetry (2.22). One finds that $R(\omega)$ and $\mathcal{R}$ transform as ${ }^{4}$

$$
\begin{aligned}
\delta_{\Lambda} R(\omega)_{\hat{a} \hat{b} \hat{c} \hat{d}} & =2 \mathcal{D}_{[\hat{a}} \Lambda_{\hat{b}] \mid \hat{c} \hat{d}}+T_{\hat{a} \hat{b}} \Lambda_{\hat{e} \mid \hat{c} \hat{d}}+\frac{1}{2} \Lambda_{\mid \hat{a} \hat{b}}^{\hat{e}} \omega_{\hat{e} \hat{c} \hat{d}}-\frac{1}{2} \Lambda_{\mid \hat{c} \hat{d}}^{\hat{e}} \omega_{\hat{e} \hat{a} \hat{b}}, \\
\delta_{\Lambda} \mathcal{R}_{\hat{a} \hat{b} \hat{c} \hat{d}} & =\mathcal{D}_{[\hat{a}} \Lambda_{\hat{b}] \mid \hat{c} \hat{d}}+\mathcal{D}_{[\hat{c}} \Lambda_{\hat{d}] \mid \hat{a} \hat{b}}+\frac{1}{2} T_{\hat{a} \hat{b}} \hat{e} \Lambda_{\hat{e} \mid \hat{c} \hat{d}}+\frac{1}{2} T_{\hat{c} \hat{d}}^{\hat{e}} \Lambda_{\hat{e} \mid \hat{a} \hat{b}} .
\end{aligned}
$$

Clearly, only a few components of $\mathcal{R}_{\hat{a} \hat{b} \hat{c} \hat{d}}$ are $\Lambda$-invariant. These are reduced further when one considers the torsion Bianchi identities, which read

$$
\begin{aligned}
\mathcal{D}_{[\hat{a}} T_{\hat{b} \hat{c} \hat{d}]} & =-\frac{3}{4} T_{[\hat{a} \hat{b}}^{\hat{e}} T_{\hat{c} \hat{d}] \hat{e}}+\frac{3}{2} \mathcal{R}_{[\hat{a} \hat{b} \hat{c} \hat{d}]}, \\
2 \mathcal{D}_{[\hat{a}} T_{\hat{b}]} & =-T_{\hat{a} \hat{b}} T_{\hat{c}}-\mathcal{D}^{\hat{c}} T_{\hat{c} \hat{a} \hat{b}}+\widehat{\mathcal{R}}_{\hat{a} \hat{b}}, \\
\mathcal{D}^{\hat{a}} T_{\hat{a}} & =-\frac{1}{2} T^{\hat{a}} T_{\hat{a}}+\frac{1}{12}\left(T_{\hat{a} \hat{b} \hat{c}}\right)^{2}-\frac{1}{2} \mathcal{R}_{\hat{a} \hat{b}} \hat{a} \hat{b}
\end{aligned}
$$

where $\widehat{\mathcal{R}}_{\hat{a} \hat{b}}$ is an antisymmetric tensor given by

$$
\widehat{\mathcal{R}}_{\hat{a} \hat{b}}=D^{\hat{c}} \omega_{\hat{c} \hat{a} \hat{b}}+F^{\hat{c}} \omega_{\hat{c} \hat{a} \hat{b}}+2 R(\omega)_{\hat{d}[\hat{a} \hat{b}]} \hat{d} .
$$

Setting $T=0$ above, one can show that the only invariant components of $\mathcal{R}$ are

$$
\mathcal{R}=\mathcal{R}_{a b}{ }^{a b}=-\mathcal{R}_{\overline{a b}}{ }^{\overline{a b}}, \quad \mathcal{R}_{a \bar{b}}=\mathcal{R}_{a c \bar{b}}{ }^{c}=-\mathcal{R}_{a \bar{c} \bar{b}}{ }^{\bar{c}} .
$$

With these ingredients, a two-derivative action for DFT can be written down in the absence of a cosmological constant:

$$
S=\int \mathrm{d}^{D} x \mathrm{~d}^{D} \tilde{x} \phi \mathcal{R}
$$

where $\phi=e^{-2 d}$ is the dilaton. The two invariants (2.30) have a simple interpretation in terms of this action. The first is the equation of motion of the dilaton and analogous to the Ricci scalar in general relativity. The second is the analogue of the Einstein tensor, being the equation of motion of the generalized vielbein. These two objects correspond to two of the linearized invariants one can construct in DFT, as noted already by Siegel in [2]. An additional possible third invariant was noted by Siegel, with indices corresponding to a mixed Riemann tensor $\mathcal{R}_{a b \overline{c d}}$. We will comment briefly about this object shortly.

\footnotetext{
${ }^{4}$ We have exhibited the torsion tensor $T$ even though we have set it to zero. This is because it will not vanish in the supersymmetric case.
} 


\subsection{Extending the geometry}

Up until this point, we have mainly been reviewing the frame formulation of DFT. Now we will begin to make some changes. Recall that the spin connection $\omega$ was introduced to gauge the $\lambda$ gauge symmetry of the vielbein. This had the effect of removing unphysical degrees of freedom; or to put it another way, in considering only gauge-covariant quantities, we are guaranteed that only physical degrees of freedom will appear. In a similar manner, we can ask to gauge the $\Lambda$ shift symmetry of the spin connection itself.

Naturally we should introduce new connections $h_{\hat{m} b \mid c d}$ and $h_{\hat{m} \bar{b} \mid \overline{c d}}$ that transform covariantly under diffeomorphisms and Lorentz transformations, but under $\Lambda$ gauge transformations as

$$
\delta_{\Lambda} h_{\hat{m} b \mid c d}=\mathcal{D}_{\hat{m}} \Lambda_{b \mid c d}, \quad \delta_{\Lambda} h_{\hat{m} \bar{b} \mid \overline{c d}}=\mathcal{D}_{\hat{m}} \Lambda_{\bar{b} \mid \overline{c d}} .
$$

Evidently, we can eliminate a good portion of the $\Lambda$ transformation in (2.27) by defining

$$
R(\omega, h)_{\hat{a} \hat{b} \hat{c} \hat{d}}=R(\omega)_{\hat{a} \hat{b} \hat{c} \hat{d}}-h_{\hat{a} \hat{b} \mid \hat{c} \hat{d}}+h_{\hat{b} \hat{a} \mid \hat{c} \hat{d}} .
$$

Now one can check that under the $\Lambda$ transformation and the anomalous Lorentz transformation,

$$
\left(\delta_{\Lambda}+\Delta_{\lambda}\right) R(\omega, h)_{\hat{a} \hat{b} \hat{c} \hat{d}}=\mathcal{T}_{\hat{a} \hat{b}}^{\hat{e}} \Lambda_{\hat{e} \mid \hat{c} \hat{d}}+\frac{1}{2}\left(\Lambda_{\mid \hat{a} \hat{b}}^{\hat{b}}-D^{\hat{e}} \lambda_{\hat{a} \hat{b}}\right) \omega_{\hat{e} \hat{c} \hat{d}}-\frac{1}{2}\left(\Lambda_{\mid \hat{c} \hat{d}}^{\hat{e}}-D^{\hat{e}} \lambda_{\hat{c} \hat{d}}\right) \omega_{\hat{e} \hat{a} \hat{b}} .
$$

The second and third terms are pairwise antisymmetric in $\hat{a} \hat{b}$ and $\hat{c} \hat{d}$, and so are eliminated when one builds $\mathcal{R}_{\hat{a} \hat{b} \hat{c} \hat{d}}(\omega, h)$,

$$
\left(\delta_{\Lambda}+\Delta_{\lambda}\right) \mathcal{R}(\omega, h)_{\hat{a} \hat{b} \hat{c} \hat{d}}=\frac{1}{2} \mathcal{T}_{\hat{a} \hat{b}}^{\hat{e}} \Lambda_{\hat{e} \mid \hat{c} \hat{d}}+\frac{1}{2} \mathcal{T}_{\hat{c} \hat{d}} \hat{e} \Lambda_{\hat{e} \mid \hat{a} \hat{b}}
$$

Observe that the $\Lambda$ transformation acts covariantly here: it "rotates" the symmetrized curvature tensor back into the torsion tensor — which in our case vanishes.

Rather than build $\mathcal{R}_{\hat{a} \hat{b} \hat{c} \hat{d}}$ by brute-force pairwise symmetrizing the indices, we can instead introduce yet another field that does this job automatically. Poláček and Siegel showed that if one extends the space of generalized coordinates to include local Lorentz coordinates $y^{a b}$ and $y^{\overline{a b}}$ (along with their duals to maintain the doubled geometry), the spin connection is naturally incorporated into the vielbein [31]. One also gets for free a new field, which we denote $p_{\hat{a} \hat{b} \hat{c} \hat{d}}$, which is pairwise antisymmetric and with both pairs valued in the two Lorentz groups. While Poláček and Siegel did not consider the connection $h$, it is straightforward to extend their analysis to include it, or even a completely arbitrary set of gauge connections. We present this analysis in appendix B.

The upshot is that one can directly build a covariant curvature tensor

$$
\mathcal{R}_{\hat{a} \hat{b} \hat{c} \hat{d}}:=R(\omega, h, p)_{\hat{a} \hat{b} \hat{c} \hat{d}}=R(\omega)_{\hat{a} \hat{b} \hat{c} \hat{d}}-h_{\hat{a} \hat{b} \mid \hat{c} \hat{d}}+h_{\hat{b} \hat{a} \mid \hat{c} \hat{d}}-p_{\hat{a} \hat{b} \hat{b} \hat{d}}
$$

with $p$ transforming as

$$
\left(\delta_{\Lambda}+\Delta_{\lambda}\right) p_{\hat{a} \hat{b} \hat{c} \hat{d}}=\frac{1}{2}\left(\Lambda_{\mid \hat{a} \hat{b}}^{\hat{e}}-D^{\hat{e}} \lambda_{\hat{a} \hat{b}}\right) \omega_{\hat{e} \hat{c} \hat{d}}-\frac{1}{2}\left(\Lambda_{\mid \hat{c} \hat{d}}^{\hat{e}}-D^{\hat{e}} \lambda_{\hat{c} \hat{d}}\right) \omega_{\hat{e} \hat{a} \hat{b}}
$$

so that only the torsion term survives in (2.34). 
We have just introduced a slew of new degrees of freedom, so we had better be able to constrain the curvature tensor to eliminate them. First, we can entirely fix $p_{\hat{a} \hat{b} \hat{c} \hat{d}}$ by enforcing that $\mathcal{R}_{\hat{a} \hat{b} \hat{c} \hat{d}}$ is pairwise symmetric. In other words, we choose $p$ so that $R(\omega, h, p)$ is identified with the pairwise symmetrized $\mathcal{R}(\omega, h)$ curvature.

Next, one can fix a large portion of $h_{\hat{m} \hat{b} \mid \hat{c} \hat{d}}$ by eliminating as many pieces of $\mathcal{R}_{\hat{a} \hat{b} \hat{c} \hat{d}}$ as possible. This is a straightforward group theory question: what representations of $h_{\hat{a} \hat{b} \mid \hat{c} \hat{d}}$ survive in the projection $h_{[\hat{a} \hat{b}] \mid c \hat{d}}+h_{[\hat{c} \hat{d}] \mid \hat{a} \hat{b}}$ ? In terms of traceless Young tableaux, $h_{\hat{a} \hat{b} \mid \hat{c} \hat{d}}$ can be decomposed as

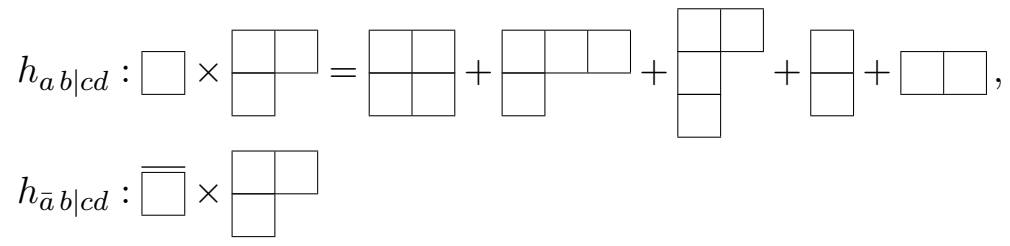

with similar expressions for $h_{a \bar{b} \mid \overline{c d}}$ and $h_{\overline{a b}} \mid \overline{c d}$. The pairwise symmetrized Riemann tensor decomposes as

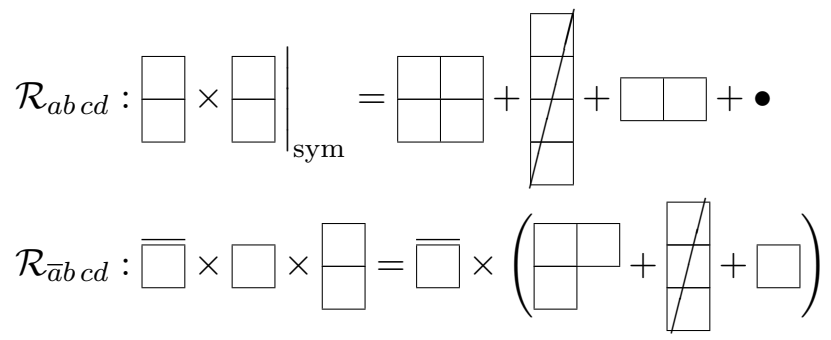

where we have crossed out the final representations that are killed by the constraint $\mathcal{R}_{[\hat{a} \hat{b} \hat{c} \hat{d}]}=0$, which is the Bianchi identity (2.28a) in the absence of torsion. ${ }^{5}$ (We do not list $\mathcal{R}_{\overline{a b} c d}$ above because it is constrained to vanish from this Bianchi identity.)

Comparing (2.38) with (2.40), it is clear that the $\square$ and $\square$ representations of $h_{a b \mid c d}$ can be used to fix the corresponding representations of $\mathcal{R}_{a b c d}$ to zero. Similarly, the single representation of $h_{\bar{a} b \mid c d}$ can be used to fix that representation of $\mathcal{R}_{\bar{a} b c d}$. Similar comments pertain to the barred/unbarred versions.

Taking into account the other Bianchi identities (2.28b) and (2.28c) in the absence of torsion, it isn't hard to see that we have eliminated all but the physical components of the Riemann tensor given in (2.30). The remaining singlet of $\mathcal{R}_{a b c d}$ is related by (2.28c) to the singlet of $\mathcal{R}_{\overline{a b} \overline{c d}}$. Similarly, the $(1,1)$ tensor of $\mathcal{R}_{\bar{a} b c d}$ is related to that of $\mathcal{R}_{a \bar{b}} \overline{c d}$ by $(2.28 \mathrm{~b})$. Recall there was a third linearized invariant discussed by Siegel in [2]. This is nothing but the tensor $p_{a b \overline{c d}}$; its non-covariance (2.37) appears only at second order.

Naturally, the above process turns out to leave certain representations in $h$ unfixed, specifically the $\square \square+\square+\square$ representations of $h_{a b \mid c d}$ and their barred versions. If we gauge these local symmetries, the process may continue. In fact, it would seem we must gauge these local symmetries in order for the algebra to close. The reason is that the

\footnotetext{
${ }^{5}$ We emphasize that the equations $(2.28 \mathrm{a})$ and $(2.28 \mathrm{c})$ continue to hold with the new $\mathcal{R}$ above, due to the symmetry properties of $h$ and $p$. The curvature $\widehat{\mathcal{R}}_{\hat{a} \hat{b}}$ in $(2.28 \mathrm{~b})$ develops a new interpretation, which we discuss in appendix B.
} 
commutator of two local $\Lambda_{a \mid b c}$ transformations must close on a new generator (or vanish). From (2.32) one can compute that

$$
\left[\delta_{\Lambda_{2}}, \delta_{\Lambda_{1}}\right] h_{a, b \mid c d}=-2 \Lambda_{[2 a b}^{e} \Lambda_{1] e c d}+2 \Lambda_{[2 a c}^{e} \Lambda_{1] b d e}-2 \Lambda_{[2 a d}^{e} \Lambda_{1] b c e} .
$$

A laborious computation shows that the right-hand side involves only the three representations $\square+\square+\boxminus$. In other words, closure of the algebra requires that we now gauge the shift symmetry in $h$. This seems likely to continue ad infinitum, and the local gauge symmetry one generates seems to be closely related to the Maxwell $\infty$ algebra.

We elaborate on this further in appendix $\mathrm{C}$ and in section 4.3 for the supersymmetric case, but let us briefly summarize the situation here. The Maxwell $1_{\infty}$ algebra is the free Lie algebra generated by $P_{a}$, combined with the Lorentz generator [32,33]. Taking $P_{a}$ to have dimension 1 , we define generators of positive dimension by

$$
\left[P_{a}, P_{b}\right]=Y_{a b}, \quad\left[P_{a}, Y_{b c}\right]=Y_{a, b c}, \quad \cdots
$$

The generator $Y_{a b}$ is antisymmetric, and the generator $Y_{a, b c}$ lies in the reducible hook representation, since the Jacobi identity of three $P$ 's leads to $Y_{[a, b c]}=0$. We will make a further restriction for what we call the Maxwell $\infty$ algebra that differs from [32, 33]: we restrict $Y_{a, b c}$ to be traceless, and denote it $Y_{a \mid b c}$. Physically this can be understood if we interpret $P_{a}$ in (2.43) to be the covariant derivative in a geometry with an on-shell Maxwell field strength. Then $Y_{a b}$ is $-\boldsymbol{F}_{a b}$ and then $Y_{a \mid b c}$ corresponds to its covariant derivative modulo the Bianchi identity and the equation of motion.

Now it follows that at dimension 4 ,

$$
\begin{aligned}
{\left[Y_{a b}, Y_{c d}\right] } & =Y_{a b, c d}, \\
{\left[P_{a}, Y_{b \mid c d}\right] } & =Y_{a|b| c d}+\left[\frac{3}{4} Y_{a b, c d}+\frac{3}{8} \eta_{a c} Y_{b}{ }^{e}, d e\right]_{b \mid c d} .
\end{aligned}
$$

The first equation defines $Y_{a b, c d}$, so it is in a reducible representation. In the second equation, $Y_{a|b| c d}$ is in an irreducible representation, which is symmetric in $a b$, antisymmetric in $c d$, traceless, and vanishing when any three indices are antisymmetrized. The Jacobi identity guarantees these are the only representations. In terms of traceless Young tableaux, these are

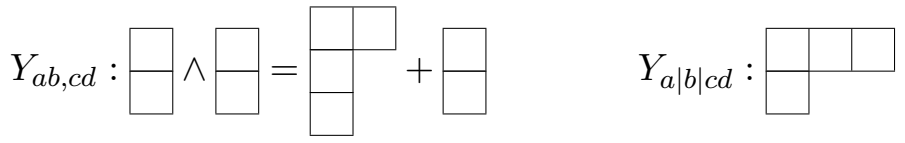

In terms of operators, $Y_{a b, c d}$ is $\left[\boldsymbol{F}_{a b}, \boldsymbol{F}_{c d}\right]$, so if we keep this generator, we are really working with non-abelian Yang-Mills operators. The second generator corresponds to two symmetrized covariant derivatives of the field strength, modulo terms that involve the Bianchi identity or the equation of motion.

The representations we are encountering match so far the representations we seek for the extension of the local algebra of bosonic double field theory. Unfortunately, the generators $Y$ we are discussing have the wrong dimension and their algebra is not the same as the 
local algebra of $\Lambda$ transformations. The solution is to recall in the Poláček-Siegel formalism that for every generator that we wish to gauge, we must introduce a dual generator that is paired with it. The $Y$ 's discussed above are exactly these dual generators. The algebra we are encountering can be embedded in the Poláček-Siegel extension of the Maxwell M $_{\infty}$ algebra discussed above. The resulting infinite algebra involves generators (and similarly for barred indices)

\begin{tabular}{l|l|c|c|c|c|c|c|c|c} 
dimension & $\cdots$ & -2 & -1 & 0 & +1 & +2 & +3 & +4 & $\cdots$ \\
\hline generator & $\cdots$ & $L_{a b, c d}, L_{a|b| c d}$ & $K_{a \mid b c}$ & $M_{a b}$ & $P_{a}$ & $\widetilde{M}^{a b}$ & $\widetilde{K}^{a \mid b c}$ & $\widetilde{L}^{a b, c d}, \widetilde{L}^{a|b| c d}$ & $\cdots$
\end{tabular}

Up to normalizations, the $Y$ generators defined previously have been renamed $\widetilde{M}, \widetilde{K}$, etc. to emphasize that they are the duals of generators of non-positive dimension. We have denoted the shift generator of the $\omega$ connection by $K$ and the shift generators of the $h$ connection by $L$ for convenience. We emphasize that $L_{a b, c d}$ is reducible. For later use, we denote the subalgebra of non-positive generators by

$$
\widehat{\mathrm{SO}}(D-1,1)=\left\{M_{a b}, K_{a \mid b c}, L_{a b, c d}, L_{a|b| c d}, \cdots\right\} .
$$

The generators and their duals are related by an infinite dimensional invertible metric $\eta$, which is the metric on the Poláček-Siegel megaspace. ( $P_{a}$ is its own dual.) The metric $\eta$ is defined in the obvious way by products of $\eta_{a b}$ with unit normalization. With this generator, one can write the full algebra of generators $X_{\mathcal{A}}$ schematically in terms of a commutator and a symmetric metric,

$$
\left[X_{\mathcal{A}}, X_{\mathcal{B}}\right]=-f_{\mathcal{A B}}{ }^{\mathcal{C}} X_{\mathcal{C}}, \quad\left\langle X_{\mathcal{A}} \mid X_{\mathcal{B}}\right\rangle=\eta_{\mathcal{A B}}
$$

with the requirement that $f_{\mathcal{A B C}}=f_{\mathcal{A B}}{ }^{\mathcal{D}} \eta_{\mathcal{D C}}$ is totally antisymmetric (which follows by ad-invariance of the metric). This allows one to use the algebra of positive dimension generators to "reflect" onto the algebra of negative dimension generators. For example,

$$
\left[P_{a}, P_{b}\right]=-2 \widetilde{M}_{a b} \quad \Longrightarrow \quad\left[M_{c b}, P_{a}\right]=\eta_{b a} P_{c}-\eta_{c a} P_{b}
$$

To avoid needless repetition, we will postpone further details until the supersymmetric case.

Another point to emphasize is that the kind of extension to $\omega$ that we postulate here is very similar to how conformal gravity extends Poincaré gravity. In conformal gravity, one introduces not just a spin connection $\omega_{m a b}$ but also a Weyl connection $b_{m}$ to gauge dilatations. The vanishing torsion condition determines $\omega_{m a b}$ but not $b_{m}$. This suggests a new local symmetry, and indeed the new symmetry one encounters is the special conformal transformation $K_{a}$, under which $b$ transforms linearly, $\delta b_{m}=e_{m}{ }^{a} \Lambda_{a}$. One is forced to introduce a new gauge field $f_{m a}$, which can be constrained to eliminate all parts of the Lorentz curvature except for the Weyl tensor. The behavior we are postulating for bosonic DFT is quite similar, except that the local gauge symmetries do not terminate at $K$.

It may seem apparent to the reader that in the bosonic case there is no necessity to introduce the local $\Lambda_{a \mid b c}$ symmetry or its corresponding gauge field $h_{\hat{m} a \mid b c}$. This is true. 
But when we move on to the supersymmetric case, we will find that the local tangent space symmetry should be extended beyond the doubled Lorentz group and closure of those transformations will inevitably lead to the appearance of the $\Lambda$ shift symmetry and its supersymmetric siblings.

\section{Double field theory in superspace}

\subsection{Elements of component supersymmetric DFT}

$\mathcal{N}=1$ supersymmetric DFT was constructed independently by Hohm and Kwak [12] (to second order in fermions) and by Jeon, Lee, and Park [13] (to all orders). The conventions in these two approaches differ somewhat. We will more closely follow Hohm and Kwak [12], although we will exchange the role of the left and right Lorentz groups, along with a number of other minor alterations.

The extension to bosonic DFT involves introducing a gravitino $\Psi_{\bar{a}}{ }^{\alpha}$ and a dilatino $\chi_{\alpha}$. Roughly speaking, the gravitino is the superpartner to the doubled vielbein and the dilatino is the superpartner to the dilaton. The index $\alpha$ is a Majorana spinor index of $\operatorname{SO}(9,1)_{L}$, and the gravitino and dilatino are of opposite chirality. The vector index $\bar{a}$ on the gravitino belongs to $\mathrm{SO}(9,1)_{R}$, so the gravitino transforms as a vector under one of the groups and a spinor under the other. As usual, the supersymmetry parameter $\epsilon^{\alpha}$ is of the same chirality as the gravitino.

In our conventions, the supersymmetry transformation of the vielbein reads

$$
\delta V_{\hat{m}}^{a}=V_{\hat{m}}{ }^{\bar{b}} J_{\bar{b}^{a}}, \quad \delta V_{\hat{m}}{ }^{\bar{a}}=V_{\hat{m}}^{b} J_{b}{ }^{\bar{a}}, \quad J_{\bar{b} a}=-J_{a \bar{b}}=-\kappa \epsilon^{\alpha}\left(\gamma_{a}\right)_{\alpha \beta} \Psi_{\bar{b}}^{\beta},
$$

and for the dilatino,

$$
\delta \phi=-\kappa \phi \epsilon^{\alpha} \chi_{\alpha}
$$

In these expressions, $\kappa$ is a dimensionless number that normalizes $\epsilon^{\alpha}$. In order to match 10D $\mathcal{N}=1$ supergravity with conventional normalizations, $\kappa$ involves an inconvenient factor of $\sqrt{2}$, so we have found it easier to leave this number a variable for now. The fermions transform (to lowest order) as

$$
\delta \Psi_{\bar{a}}^{\alpha}=\mathcal{D}_{\bar{a}} \epsilon^{\alpha}+\cdots, \quad \delta \chi_{\alpha}=\left(\gamma^{a}\right)_{\alpha \beta} \mathcal{D}_{a} \epsilon^{\beta}+\cdots .
$$

In contrast to Hohm and Kwak [12], $\mathcal{D}_{\hat{a}}$ is defined as $V_{\hat{a}}^{\hat{m}} \partial_{\hat{m}}$ without a factor of $\sqrt{2}$.

A key feature of supersymmetric DFT is that both fermions are connections: they each transform into a derivative of the supersymmetry parameter. This implies that in any superspace formulation they ought to be encoded in the supervielbein.

\subsection{Siegel's superspace DFT}

Let us now construct double field theory in $\mathcal{N}=1$ superspace. We begin with a review of Siegel's early construction [2], with some minor modifications to conventions. While Siegel also treated heterotic double field theory in the abelian limit, we restrict our attention to the case without vector multiplets for simplicity. The basic details are also 
identical to Cederwall's discussion of double supergeometry [20], except with half as many fermionic coordinates.

In analogy to conventional $\mathrm{O}(D, D)$ double field theory, let us introduce $\operatorname{OSp}(D, D \mid 2 s)$ double field theory with $2 D$ bosonic coordinates and $2 s$ fermionic ones. We will really only be concerned with $D=10$ and $s=16$. Collectively, we denote these supercoordinates by $z^{\mathcal{M}}$. Superdiffeomorphisms have an $\operatorname{OSp}(D, D \mid 2 s) \times \mathbb{R}^{+}$structure, and act on a vector $W^{\mathcal{M}}$ of weight $w$ as

$$
\mathbb{L}_{\xi}^{(w)} W^{\mathcal{M}}=\xi^{\mathcal{N}} \partial_{\mathcal{N}} W^{\mathcal{M}}-\mathcal{W}^{\mathcal{N}}\left(\partial_{\mathcal{N}} \xi^{\mathcal{M}}-\partial^{\mathcal{M}} \xi_{\mathcal{N}}(-1)^{n m}\right)+w \partial_{\mathcal{N}} \xi^{\mathcal{N}} W^{\mathcal{M}}(-1)^{n}
$$

where the grading $(-1)^{n m}$ is -1 if both $\mathcal{N}$ and $\mathcal{M}$ are fermionic and +1 otherwise. An index $\mathcal{M}$ is raised or lowered with the canonical orthosymplectic element $\eta$ of $\operatorname{OSp}(D, D \mid 2 s)$, with the usual NW-SE convention, i.e.

$$
W^{\mathcal{M}}=\eta^{\mathcal{M N}} W_{\mathcal{N}}, \quad W_{\mathcal{M}}=W^{\mathcal{N}} \eta_{\mathcal{N} \mathcal{M}}
$$

The metric $\eta$ itself is graded symmetric, $\eta_{\mathcal{M N}}=\eta_{\mathcal{N M}}(-1)^{n m}$. Because of the grading, $\eta_{\mathcal{M N}}$ is not quite the inverse of $\eta^{\mathcal{M N}}$; instead, one finds

$$
\eta^{\mathcal{M P}} \eta_{\mathcal{P N}}=\delta_{\mathcal{N}^{\mathcal{M}}}(-1)^{n m}
$$

Closure of the algebra of superdiffeomorphisms requires the section condition

$$
\eta^{\mathcal{M N}} \partial_{\mathcal{N}} \otimes \partial_{\mathcal{M}}=0
$$

In the standard coordinate basis, the coordinates are denoted $z^{\mathcal{M}}=\left(x^{\mathrm{m}}, \tilde{x}_{\mathrm{m}}, \theta^{\mu}, \tilde{\theta}_{\mu}\right)$ and $\eta$ is given by

$$
\eta^{\mathcal{M N}}=\left(\begin{array}{cccc}
0 & \delta^{\mathrm{m}}{ }_{\mathrm{n}} & 0 & 0 \\
\delta_{\mathrm{m}}{ }^{\mathrm{n}} & 0 & 0 & 0 \\
0 & 0 & 0 & \delta^{\mu}{ }_{\nu} \\
0 & 0 & -\delta_{\mu}{ }^{\nu} & 0
\end{array}\right), \quad \eta_{\mathcal{M N}}=\left(\begin{array}{cccc}
0 & \delta_{\mathrm{m}}{ }^{\mathrm{n}} & 0 & 0 \\
\delta^{\mathrm{m}}{ }_{\mathrm{n}} & 0 & 0 & 0 \\
0 & 0 & 0 & \delta_{\mu}{ }^{\nu} \\
0 & 0 & -\delta^{\mu}{ }_{\nu} & 0
\end{array}\right) .
$$

The section condition can then be solved by restricting fields and parameters to depend only on $z^{\mathrm{M}}=\left(x^{\mathrm{m}}, \theta^{\mu}\right)$, the coordinates of $10 \mathrm{D} \mathcal{N}=1$ superspace. It will often be useful to collectively denote the doubled bosonic coordinates by $x^{\hat{m}}=\left(x^{\mathrm{m}}, \tilde{x}_{\mathrm{m}}\right)$ and the fermionic ones by $\theta^{\hat{\mu}}=\left(\theta^{\mu}, \tilde{\theta}_{\mu}\right)$. It will also be useful occasionally to use left/right-moving coordinates $x^{m}$ and $x^{\bar{m}}$; these are defined exactly as in the bosonic case. There are no fermionic analogues to the left or right-moving bosonic coordinates, partly because $\theta$ and $\tilde{\theta}$ have different engineering dimension. If $x$ and $\tilde{x}$ are both taken to have dimension -1 , and if $\theta$ has dimension $-\frac{1}{2}$ (to allow a conventional $\mathcal{N}=1$ superspace to emerge), then the $\operatorname{OSp}(D, D \mid 2 s)$ structure forces $\tilde{\theta}$ to have dimension $-\frac{3}{2}[20]$.

The supervielbein is naturally taken as a weighted element of $\operatorname{OSp}(D, D \mid 2 s) \times \mathbb{R}^{+}$. As in the bosonic case, it is more convenient to split the supervielbein into an $\operatorname{OSp}(D, D \mid 2 s)$ element, which we denote $\mathcal{V}_{\mathcal{M}}{ }^{\mathcal{A}}$, and the superdilaton $\Phi .{ }^{6}$ These transform respectively as

$$
\begin{aligned}
\delta \mathcal{V}_{\mathcal{M}}{ }^{\mathcal{A}} & =\mathbb{L}_{\xi} \mathcal{V}_{\mathcal{M}}{ }^{\mathcal{A}}=\xi^{\mathcal{N}} \mathcal{V}_{\mathcal{M}}{ }^{\mathcal{A}}+\left(\partial_{\mathcal{M}} \xi^{\mathcal{N}}-\partial^{\mathcal{N}} \xi_{\mathcal{M}}(-1)^{n m}\right) \mathcal{V}_{\mathcal{N}^{\mathcal{A}}} \\
\delta \Phi & =\mathbb{L}_{\xi}^{(+1)} \Phi=\xi^{\mathcal{N}} \partial_{\mathcal{N}} \Phi+\partial_{\mathcal{N}} \xi^{\mathcal{N}} \Phi(-1)^{n}
\end{aligned}
$$

\footnotetext{
${ }^{6}$ Siegel denoted the superdilaton by $\Phi^{2}$ so that his $\Phi$ has weight $\frac{1}{2}$.
} 
It is going to turn out that the superdilaton $\Phi$ differs from the component dilaton $\phi=e^{-2 d}$, so we have used a different name for it. The index $\mathcal{A}$ decomposes into a left sector $A$ and a right sector $\bar{A}$, with

$$
W^{\mathcal{A}}=\left(W^{A}, W^{\bar{A}}\right), \quad W^{A}=\left(W^{a}, W^{\alpha}, W_{\alpha}\right), \quad W^{\bar{A}}=\left(W^{\bar{a}}\right) .
$$

Siegel identified these sectors as the left and right-handed sectors of oscillators of the affine Lie algebra of the superstring in the Hamiltonian framework. Here $a$ and $\bar{a}$ are the tangent space indices associated with the local $\mathrm{O}(D-1,1)_{L}$ and $\mathrm{O}(D-1,1)_{R}$ actions on the bosonic DFT vielbein. In the supersymmetric case, $a$ is extended to include spinors of both chiralities. The tangent space metric and its inverse are

$$
\eta^{\mathcal{A B}}=\left(\begin{array}{ccc|c}
\eta^{a b} & 0 & 0 & 0 \\
0 & 0 & \delta^{\alpha}{ }_{\beta} & 0 \\
0 & -\delta_{\alpha}{ }^{\beta} & 0 & 0 \\
\hline 0 & 0 & 0 & -\eta^{\overline{a b}}
\end{array}\right), \quad \eta_{\mathcal{A B}}=\left(\begin{array}{ccc|c}
\eta_{a b} & 0 & 0 & 0 \\
0 & 0 & \delta_{\alpha}{ }^{\beta} & 0 \\
0 & -\delta^{\alpha}{ }_{\beta} & 0 & 0 \\
\hline 0 & 0 & 0 & -\eta_{\overline{a b}}
\end{array}\right)
$$

where the horizontal and vertical lines emphasize the split between the left and right sectors. The metrics $\eta^{a b}$ and $\eta^{\overline{a b}}$ both describe $\mathrm{SO}(D-1,1)$ (with mostly positive signature) and are used to raise and lower their respective indices. The condition that the supervielbein is a group element amounts to

$$
\mathcal{V}_{\mathcal{A}}^{\mathcal{M}}=\eta^{\mathcal{M N}} \mathcal{V}_{\mathcal{N}}{ }^{\mathcal{B}} \eta_{\mathcal{B} \mathcal{A}}(-1)^{a m}
$$

The right tangent space is $\mathrm{O}(D-1,1)_{R}$ as in the bosonic theory, whereas the left tangent space must be a subgroup of $\operatorname{OSp}(D-1,1 \mid 2 s)$. We will elaborate on the choice of this tangent space momentarily.

Although it is natural to group together the left sector tangent space indices, we will usually group bosonic indices together, so that $W^{\mathcal{A}}=\left(W^{a}, W^{\bar{a}}, W^{\alpha}, W_{\alpha}\right)$, to be more in line with how we have ordered our coordinates. Using the same rules for raising and lowering indices as before, one finds that

$$
W^{\mathcal{A}}=\left(W^{a}, W^{\bar{a}}, W^{\alpha}, W_{\alpha}\right) \quad \Longrightarrow \quad W_{\mathcal{A}}=\left(W_{a},-W_{\bar{a}},-W_{\alpha}, W^{\alpha}\right) .
$$

Therefore, when specifying explicit elements, one must be careful to emphasize whether the indices are taken to be vectors or covectors because of the signs that are introduced above.

Just as one may use left/right coordinates $z^{\mathcal{M}}=\left(x^{m}, x^{\bar{m}}, \theta^{\mu}, \theta_{\mu}\right)$ to match the left/right tangent space, one may occasionally wish to put the tangent space in its standard toroidal form, where $W^{\mathcal{A}}=\left(W^{\mathrm{a}}, W_{\mathrm{a}}, W^{\alpha}, W_{\alpha}\right)$, in line with the standard toroidal coordinates $z^{\mathcal{M}}=\left(x^{\mathrm{m}}, \tilde{x}_{\mathrm{m}}, \theta^{\mu}, \tilde{\theta}_{\mu}\right)$. We use lower case Roman font indices a, $\mathrm{b}, \cdots$ to denote the tangent frame bosonic indices in the standard basis.

\subsection{Determining the tangent space}

In order to motivate how we will fix the tangent space in superspace, let's return to bosonic DFT and discuss how one may go about determining its physical content. We will take a 
more elaborate approach than is actually required, but this approach will generalize more straightforwardly to superspace.

Working for the moment in the standard toroidal basis for both the coordinate and tangent space indices, the vielbein can be decomposed as

$$
V_{\hat{m}}^{\hat{a}}=\left(\begin{array}{ll}
1 & b \\
0 & 1
\end{array}\right) \times\left(\begin{array}{cc}
e & 0 \\
0 & e^{-T}
\end{array}\right) \times\left(\begin{array}{ll}
1 & 0 \\
c & 1
\end{array}\right)
$$

where $b=b_{\mathrm{mn}}, e=e_{\mathrm{m}}{ }^{\mathrm{a}}$, and $c=c^{\mathrm{ab}}$. It is easy to check that this is an $\mathrm{O}(D, D)$ element by computing $V^{-1}=\eta V^{T} \eta$. This decomposition can be understood as arising by first decomposing the generators $X_{\hat{m} \hat{n}}$ of $\mathrm{O}(D, D)$ into $X^{\mathrm{mn}}, X_{\mathrm{m}}{ }^{\mathrm{n}}$, and $X_{\mathrm{mn}}$. Our $\mathrm{O}(D, D)$ and $\operatorname{OSp}(D, D \mid 2 s)$ conventions can be found in appendix A. The generators can be assigned levels $-1,0$, and +1 with respect to an outer automorphism of the algebra. The above element then corresponds to a parametrization of the group element $V$ as

$$
V=\exp \left(\frac{1}{2} b_{\mathrm{mn}} X^{\mathrm{nm}}\right) \times \exp \left(a_{\mathrm{m}}{ }^{\mathrm{n}} X_{\mathrm{n}}{ }^{\mathrm{m}}\right) \times \exp \left(\frac{1}{2} c^{\mathrm{mn}} X_{\mathrm{nm}}\right)
$$

and taking $V$ to act to the left on the coordinate representation. One identifies $e_{\mathrm{m}}{ }^{\mathrm{a}}=\exp (a)$.

In this parametrization, a general coordinate transformation with $\partial^{\mathrm{m}}=0$ transforms only $b_{\mathrm{mn}}$ and $e_{\mathrm{m}}{ }^{\mathrm{a}}$. To see this, observe that the general coordinate transformation involves

$$
\mathcal{K}_{\hat{m}}^{\hat{n}}:=\partial_{\hat{m}} \xi^{\hat{n}}-\partial^{\hat{n}} \xi_{\hat{m}}=\left(\begin{array}{cc}
\partial_{\mathrm{m}} \xi^{\mathrm{n}} & 2 \partial_{[\mathrm{m}} \xi_{\mathrm{n}]} \\
0 & -\partial_{\mathrm{n}} \xi^{\mathrm{m}}
\end{array}\right)
$$

which is an $\mathrm{O}(D, D)$ generator at levels 0 and -1 . Writing the vielbein as $V=V_{-1} V_{0} V_{+1}$ and the generator as $\mathcal{K}=\mathcal{K}_{-1}+\mathcal{K}_{0}$, a general coordinate transformation then acts as

$$
\delta_{\text {g.c. }} V=\mathcal{K} V \quad \Longrightarrow \quad \delta_{\text {g.c. }} V_{-1}=\mathcal{K}_{-1} V_{-1}+\left[\mathcal{K}_{0}, V_{-1}\right], \quad \delta_{\text {g.c. }} V_{0}=\mathcal{K}_{0} V_{0}, \quad \delta_{\text {g.c. }} V_{+1}=0
$$

which amount to the usual transformations

$$
\delta_{\text {g.c. }} b_{\mathrm{mn}}=2 \partial_{[\mathrm{m}} \xi_{\mathrm{n}]}+\partial_{\mathrm{m}} \xi^{\mathrm{p}} b_{\mathrm{pn}}-b_{\mathrm{mp}} \partial_{\mathrm{n}} \xi^{\mathrm{p}}, \quad \delta_{\text {g.c. }} e_{\mathrm{m}}{ }^{\mathrm{a}}=\partial_{\mathrm{m}} \xi^{\mathrm{n}} e_{\mathrm{n}}{ }^{\mathrm{a}}, \quad \delta_{\text {g.c. }} c^{\mathrm{ab}}=0 .
$$

The field $c^{\mathrm{ab}}$ itself can be set to zero using half of the $\mathrm{O}(D-1,1)_{L} \times \mathrm{O}(D-1,1)_{R}$ tangent space symmetry. Recall that $\mathfrak{s o}(D-1,1)_{L}+\mathfrak{s o}(D-1,1)_{R}$ is represented in the standard toroidal basis as

$$
\lambda_{\hat{a}}^{\hat{b}}=\left(\begin{array}{cc}
\lambda_{\mathrm{a}}^{\mathrm{b}} & \tilde{\lambda}_{\mathrm{ab}} \\
\tilde{\lambda}^{\mathrm{ab}} & \lambda^{\mathrm{a}}{ }_{\mathrm{b}}
\end{array}\right), \quad \lambda=\frac{1}{2}\left(\lambda_{L}+\lambda_{R}\right), \quad \tilde{\lambda}=\frac{1}{2}\left(\lambda_{L}-\lambda_{R}\right)
$$

The indices $\mathrm{a}, \mathrm{b}, \cdots$ denote vectors of the diagonal $\mathrm{SO}(D-1,1)$ transformation, corresponding to antisymmetric $\lambda_{\mathrm{ab}}$ above. The anti-diagonal transformations are generated by antisymmetric $\tilde{\lambda}_{\mathrm{ab}}$. (In both cases, we raise and lower indices with the diagonal $\eta_{\mathrm{ab}}$.) Now consider a double Lorentz transformation acting on the right of $V$. The diagonal transformations, which lie at level 0 , evidently transform $c$ as a 2 -form, $e$ as a vector on 
the right, and leave $b$ invariant, which justifies their index structures. An infinitesimal $\tilde{\lambda}$ transformation is more complicated, comprising a sum of levels -1 and +1 . Because $c^{\text {ab }}$ is situated on the far right of the group element, lying at level +1 , it can be eliminated immediately, whereas $e_{\mathrm{m}}{ }^{\mathrm{a}}$ and $b_{\mathrm{mn}}$ will transform in much more complicated ways. The explicit form of their transformations is irrelevant: we only need to know that we can impose the gauge $c=0$. This gauge will then be undisturbed both by diagonal Lorentz transformations and by general coordinate transformations obeying $\partial^{\mathrm{m}}=0$.

Returning to superspace, we introduce a decomposition of the supervielbein in direct analogy to (3.14). Group the physical coordinates together so that $z^{\mathcal{M}}=\left(z^{\mathrm{M}}, \tilde{z}_{\mathrm{M}}\right)=$ $\left(x^{\mathrm{m}}, \theta^{\mu}, \tilde{x}_{\mathrm{m}}, \tilde{\theta}_{\mu}\right)$ and similarly for the tangent space, $W^{\mathcal{A}}=\left(W^{\mathrm{A}}, W_{\mathrm{A}}\right)=\left(W^{\mathrm{a}}, W^{\alpha}, W_{\mathrm{a}}, W_{\alpha}\right)$. Here $z^{\mathrm{M}}$ is the coordinate of $10 \mathrm{D} \mathcal{N}=1$ superspace and $\mathrm{A}$ is its tangent superspace index. The supervielbein decomposes as

$$
\mathcal{V}_{\mathcal{M}}{ }^{\mathcal{A}}=\left(\begin{array}{ll}
1 & B \\
0 & 1
\end{array}\right) \times\left(\begin{array}{cc}
E & 0 \\
0 & E^{-T}
\end{array}\right) \times\left(\begin{array}{ll}
1 & 0 \\
C & 1
\end{array}\right)
$$

where $B=B_{\mathrm{MN}}(-)^{n}, E=E_{\mathrm{M}}^{\mathrm{A}}, E^{-T}=E_{\mathrm{A}}^{\mathrm{M}}(-1)^{a m+a}$ and $C=C^{\mathrm{AB}}$. This corresponds to a level decomposition with the $\operatorname{OSp}(D, D \mid 2 s)$ generator $X_{\mathcal{M N}}$ decomposed into the $\operatorname{GL}(D \mid s)$ generator $X_{\mathrm{M}}{ }^{\mathrm{N}}$ at level 0 and generators $X_{\mathrm{MN}}$ and $X^{\mathrm{MN}}$ at levels \pm 1 .

The supervielbein $E_{\mathrm{M}}{ }^{\mathrm{A}}$ and super-two-form $B_{\mathrm{MN}}$ both play natural roles in $10 \mathrm{D} \mathcal{N}=1$ superspace, and it is straightforward as in the bosonic case to show that they transform under $\tilde{z}_{\mathrm{M}}$-independent general coordinate transformations in the expected manner:

$$
\delta_{\text {g.c. }} B_{\mathrm{MN}}=2 \partial_{[\mathrm{M}} \tilde{\xi}_{\mathrm{N}]}+\partial_{\mathrm{M}} \xi^{\mathrm{P}} B_{\mathrm{PN}}-B_{\mathrm{MP}} \partial_{\mathrm{N}} \xi^{\mathrm{P}}(-1)^{n p}, \quad \delta_{\text {g.c. }} E_{\mathrm{M}}{ }^{\mathrm{A}}=\partial_{\mathrm{M}} \xi^{\mathrm{N}} E_{\mathrm{N}}{ }^{\mathrm{A}}
$$

where we have taken $\xi_{\mathcal{M}}=\left(\tilde{\xi}_{\mathrm{M}}, \xi^{\mathrm{M}}\right)$, or equivalently, $\xi^{\mathcal{M}}=\left(\xi^{\mathrm{M}}, \tilde{\xi}_{\mathrm{M}}(-)^{m}\right)$. In analogy with the bosonic case, we would like to eliminate $C^{\mathrm{AB}}$. Because this involves not only $C^{\text {ab }}$ but also $C^{\mathrm{a} \beta}$ and $C^{\alpha \beta}$, we require an enhancement of the bosonic tangent group. Using current algebra arguments, Siegel made a choice that amounts to enhancing $\mathrm{O}(D-1,1)_{L}$ to a subgroup of $\operatorname{OSp}(D-1,1 \mid 2 s)_{L}$ where the generator $\lambda_{A}{ }^{B}$ is subject to the following conditions

$$
\lambda_{\alpha}{ }^{b}=0, \quad \lambda_{\alpha}{ }^{\beta}=\frac{1}{4} \lambda^{a b}\left(\gamma_{a b}\right)_{\alpha}{ }^{\beta},
$$

with $\lambda^{\alpha \beta}, \lambda^{a b}$, and $\lambda^{a \beta}$ unconstrained. The second condition above amounts to the requirement that fermionic orthosymplectic indices transform as spinors under the $\mathrm{SO}(D-1,1)$ subgroup of $\operatorname{OSp}(D-1,1 \mid 2 s)$. In essence, the $\operatorname{OSp}(D-1,1 \mid 2 s)$ generators $M_{A B}$ are truncated to just the subalgebra involving $M_{\alpha \beta}, M_{\alpha b}$, and $M_{a b}^{\prime}=M_{a b}+\frac{1}{4}\left(\gamma_{a b}\right)_{\alpha}{ }^{\beta} M_{\beta}{ }^{\alpha}$ corresponding to generators $\lambda^{\alpha \beta}, \lambda^{a \beta}$ and $\lambda^{a b}$. As in the bosonic case, this is sufficient gauge freedom to eliminate $C^{\mathrm{AB}}$. Siegel went on in [2] to identify the generalized flux tensors (and torsion tensors) of superspace and showed which constraints led to 10D $\mathcal{N}=1$ superspace.

There is a subtlety in this approach when we compare with component supersymmetric DFT. The component dilatino, which transforms non-tensorially under supersymmetry (3.3), does not seem to have a natural interpretation as a component of a covariant torsion tensor, which is where it arises in $10 \mathrm{D} \mathcal{N}=1$ superspace. For that reason, one would expect it to 
more naturally arise in the supervielbein itself. But this appears in conflict with the simple $10 \mathrm{D} \mathcal{N}=1$ superspace reduction above, where it seems it must reside in a torsion tensor.

What seems to happen in Siegel's approach is that indeed the dilatino does appear as a dimension $\frac{1}{2}$ component of the torsion tensor. However, the torsion tensor turns out to not actually be invariant under the $\lambda_{A}{ }^{B}$ tangent space transformation discussed above. Instead, under a $\lambda_{a}{ }^{\beta}$ transformation, a dimension $\frac{1}{2}$ piece of the torsion tensor (namely, $\mathcal{T}_{\alpha \beta}{ }^{\gamma}$ ) transforms into the dimension 0 piece of the torsion tensor (namely, $\mathcal{T}_{\alpha \beta c} \propto\left(\gamma_{c}\right)_{\alpha \beta}$ ). This transformation turns out to involve only the spin-1/2 piece of $\lambda_{a}{ }^{\beta}$. This implies that $\mathcal{T}_{\alpha \beta}{ }^{\gamma}$ must carry a spin- $1 / 2$ field (identified with the dilatino) that transforms inhomogeneously under the spin- $1 / 2$ part of $\lambda_{a}{ }^{\beta}$. It is essentially a compensator field for this symmetry. This anomalous tangent space symmetry is responsible for imparting the non-tensorial supersymmetry transformation, as a SUSY transformation must be accompanied by a compensating $\lambda$ gauge transformation to retain the gauge $C^{\mathrm{AB}}=0$.

In formulating $\mathcal{N}=1$ superspace DFT in detail, we have found it more useful to take a different approach. We will eliminate the dilatino from the torsion tensor, fixing the spin-1/2 part of the $\lambda_{a}{ }^{\beta}$ transformation so that $\lambda_{a}{ }^{\beta}$ is now purely spin-3/2. Now not all of $C^{\mathrm{AB}}$ can be eliminated, and the piece that remains will be identified with the component dilatino. While this obscures the reduction to $10 \mathrm{D} \mathcal{N}=1$ superspace (which will now require a "degauging" to shift a field from the supervielbein to the torsion tensor), it significantly simplifies the superspace DFT curvatures and streamlines the reduction to the component theory. ${ }^{7}$

\section{Superspace geometry and its constraints}

Having introduced the supervielbein and its tangent space group, we can now explain the superspace geometry and give the appropriate torsion constraints.

\subsection{Generalized fluxes and their constraints}

The supervielbein $\mathcal{V}_{\mathcal{M}}{ }^{\mathcal{A}}$ and the superdilaton $\Phi$ transform under generalized diffeomorphisms as (3.9). From these fields, one can construct generalized fluxes $\mathcal{F}_{\mathcal{A B C}}$ and $\mathcal{F}_{\mathcal{A}}$ as

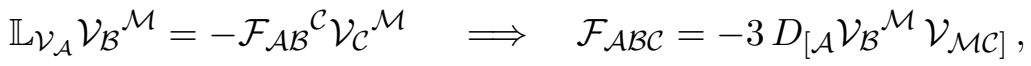

$$
\begin{aligned}
& \mathbb{L}_{\mathcal{V}_{\mathcal{A}}}^{(+1)} \Phi=\mathcal{F}_{\mathcal{A}} \Phi \quad \Longrightarrow \quad \mathcal{F}_{\mathcal{A}}=D_{\mathcal{A}} \log \Phi+\partial_{\mathcal{M}} \mathcal{V}_{\mathcal{A}}{ }^{\mathcal{M}}(-)^{a m+m}
\end{aligned}
$$

The flattened derivatives $D_{\mathcal{A}}:=\mathcal{V}_{\mathcal{A}} \mathcal{M}_{\mathcal{M}}$ here obey

$$
\left[D_{\mathcal{A}}, D_{\mathcal{B}}\right]=-\mathcal{F}_{\mathcal{A B}}{ }^{\mathcal{C}} D_{\mathcal{C}}, \quad D^{\mathcal{A}} D_{\mathcal{A}}=-\mathcal{F}^{\mathcal{A}} D_{\mathcal{A}}
$$

\footnotetext{
${ }^{7}$ Perhaps the most democratic approach would be to keep the full $\lambda_{a}{ }^{\beta}$ symmetry and show how one can both reduce to $10 \mathrm{D} \mathcal{N}=1$ superspace and also to component supersymmetric DFT depending on how the gauge is fixed. It should be possible to take the formulation we present in this paper and "regauge" it to restore the full $\lambda_{a}{ }^{\beta}$ symmetry by inserting a Stueckelberg field into the torsion tensor.
} 


\begin{tabular}{c|l}
\hline dimension & fluxes \\
\hline$-\frac{1}{2}$ & $\mathcal{F}_{\alpha \beta \gamma}=\mathcal{T}_{\alpha \beta \gamma}$ \\
\hline 0 & $\mathcal{F}_{\alpha \beta c}=\mathcal{T}_{\alpha \beta c}$ \\
& $\mathcal{F}_{\alpha \beta \bar{c}}=\mathcal{T}_{\alpha \beta \bar{c}}$ \\
\hline$\frac{1}{2}$ & $\mathcal{F}_{\alpha b \bar{c}}=\mathcal{T}_{\alpha b \bar{c}}$ \\
& $\mathcal{F}_{\alpha b c}, \mathcal{F}_{\alpha \overline{b c}}, \mathcal{F}_{\alpha \beta}{ }^{\gamma}, \mathcal{F}_{\alpha}$ \\
\hline 1 & $\mathcal{F}_{a b c}, \mathcal{F}_{a b \bar{c}}, \mathcal{F}_{a \overline{b c}}, \mathcal{F}_{\overline{a b c}}$ \\
& $\mathcal{F}_{\bar{a} \beta}{ }^{\gamma}, \mathcal{F}_{a \beta}{ }^{\gamma}$ \\
\hline$\frac{3}{2}$ & $\mathcal{F}_{a}, \mathcal{F}_{\bar{a}}$ \\
\hline 2 & $\mathcal{F}_{a b}{ }^{\gamma}, \mathcal{F}_{a \bar{b}}{ }^{\gamma}, \mathcal{F}_{\overline{a b}}^{\gamma}$ \\
\hline$\frac{5}{2}$ & $\mathcal{F}_{\alpha}{ }^{\beta \gamma}, \mathcal{F}^{\alpha}$ \\
\hline & $\mathcal{F}_{a}^{\beta \gamma}, \mathcal{F}_{\bar{a}}^{\beta \gamma}$ \\
\hline & $\mathcal{F}^{\alpha \beta \gamma}$ \\
\hline
\end{tabular}

Table 1. Generalized fluxes in superspace DFT. The lowest dimension fluxes are covariant and may be identified as torsion tensors.

These flux tensors in turn obey the following Bianchi identities:

$$
\begin{aligned}
4 D_{[\mathcal{A}} \mathcal{F}_{\mathcal{B C D}]} & =-3 \mathcal{F}_{[\mathcal{A B} \mid} \mathcal{E}^{\mathcal{E}} \mathcal{F}_{\mathcal{E} \mid \mathcal{C D}]} \\
2 D_{[\mathcal{A}} \mathcal{F}_{\mathcal{B}]} & =-\mathcal{F}_{\mathcal{A B}} \mathcal{C}_{\mathcal{C}}-D^{\mathcal{C}} \mathcal{F}_{\mathcal{C} \mathcal{B}} \\
D^{\mathcal{A}} \mathcal{F}_{\mathcal{A}} & =-\frac{1}{2} \mathcal{F}^{\mathcal{A}} \mathcal{F}_{\mathcal{A}}-\frac{1}{12} \mathcal{F}^{\mathcal{A B C}} \mathcal{F}_{\mathcal{C B} \mathcal{A}}
\end{aligned}
$$

The fluxes themselves are not invariant under the tangent space group, which we denote by $\mathcal{H}$. This group leaves the superdilaton invariant and acts on the supervielbein and fluxes as

$$
\delta \mathcal{V}_{\mathcal{A}}{ }^{\mathcal{M}}=\lambda_{\mathcal{A}}{ }^{\mathcal{B}} \mathcal{V}_{\mathcal{B}}{ }^{\mathcal{M}}, \quad \delta \mathcal{F}_{\mathcal{A B C}}=-3 D_{[\mathcal{A}} \lambda_{\mathcal{B C}]}+3 \lambda_{[\mathcal{A}}{ }^{\mathcal{D}} \mathcal{F}_{\mathcal{D} \mid \mathcal{B C}]}, \quad \delta \mathcal{F}_{\mathcal{A}}=-D^{\mathcal{B}} \lambda_{\mathcal{B} \mathcal{A}}-\mathcal{F}^{\mathcal{B}} \lambda_{\mathcal{B} \mathcal{A}}
$$

The parameter $\lambda$ belongs to the group $\mathcal{H}_{L} \times \mathrm{SO}(D-1,1)_{R}$, where $\mathcal{H}_{L}$ is a subgroup of $\operatorname{OSp}(D-1,1 \mid 2 s)_{L}$. As discussed in section 3.3, we restrict $\mathcal{H}_{L}$ so that the graded antisymmetric parameter $\lambda_{\mathcal{A B}}$ obeys

$$
\lambda_{\alpha b}=\lambda_{\alpha \beta}=0, \quad \lambda_{\alpha}{ }^{\beta}=\frac{1}{4} \lambda_{a b}\left(\gamma^{a b}\right)_{\alpha}{ }^{\beta}, \quad\left(\gamma^{a}\right)_{\alpha \beta} \lambda_{a}{ }^{\beta}=0 .
$$

In other words, the group $\mathcal{H}_{L}$ is generated by $\mathrm{SO}(D-1,1)$ transformations $\lambda_{a b}, \gamma$-traceless fermionic transformations $\lambda_{a}{ }^{\beta}$, and symmetric $\lambda^{\alpha \beta}$ transformations. We are interested in $D=10$ and $s=16$, which means $\lambda^{\alpha \beta}$ is reducible: it corresponds to a vector and a self-dual 5-form.

The generalized fluxes are grouped by dimension in table 1. Given the restrictions placed on the tangent space, some of the fluxes of low dimension are already gauge invariant 
and can be identified immediately with invariant torsion tensors $\mathcal{T}$. Let's review the constraints imposed by Siegel [2]:

- The dimension $-\frac{1}{2}$ tensor $\mathcal{T}_{\alpha \beta \gamma}$ corresponds in $10 \mathrm{D} \mathcal{N}=1$ superspace to the lowest dimension component $H_{\alpha \beta \gamma}$ of the 3 -form field strength. This is constrained to vanish in conventional superspace, and the same choice should be made here.

- At dimension 0 , one encounters $\mathcal{T}_{\alpha \beta c}$ and $\mathcal{T}_{\alpha \beta \bar{c}}$. The only sensible choice is to take $\mathcal{T}_{\alpha \beta c}=\kappa\left(\gamma_{c}\right)_{\alpha \beta}$, for $\kappa$ some numerical constant, and $\mathcal{T}_{\alpha \beta \bar{c}}=0$. These amount to the usual dimension 0 constraints, $T_{\alpha \beta}^{\mathrm{c}} \propto\left(\gamma^{\mathrm{c}}\right)_{\alpha \beta}$ and $H_{\alpha \beta \mathrm{c}} \propto\left(\gamma_{\mathrm{c}}\right)_{\alpha \beta}$ in 10D $\mathcal{N}=1$ superspace. Our choice of $\kappa$ is left unspecified, but later on we will explain how to choose it to recover conventional normalizations.

- At dimension $\frac{1}{2}$, the torsion tensor $\mathcal{T}_{\alpha b \bar{c}}$ can be shown to be $\gamma$-traceless in $\alpha b$ as a consequence of the flux Bianchi identities. This implies that $\mathcal{T}_{\alpha b \bar{c}} \propto\left(\gamma_{b}\right)_{\alpha \beta} \mathcal{W}_{\bar{c}}{ }^{\beta}$ for some superfield $\mathcal{W}_{\bar{c}}{ }^{\beta}$. There is no covariant dimension $\frac{1}{2}$ field in component supersymmetric DFT that $\mathcal{W}_{\bar{c}}{ }^{\beta}$ should be identified with, so we choose to set this field to zero. ${ }^{8}$

We summarize here the constraints we have explicitly imposed so far:

$$
\mathcal{T}_{\alpha \beta \gamma}=0, \quad \mathcal{T}_{\alpha \beta c}=\kappa\left(\gamma_{c}\right)_{\alpha \beta}, \quad \mathcal{T}_{\alpha \beta \bar{c}}=0, \quad \mathcal{T}_{\alpha b \bar{c}}=0
$$

The other geometric fluxes must be augmented by tangent space connections, as in the bosonic case, if they are to be gauge covariant.

\subsection{Tangent space connections and torsion tensors}

Following Siegel, let us introduce a tangent space connection. We denote it $\Omega_{\mathcal{A B C}}$, using the common superspace convention of denoting superfields with capital letters. This is to avoid confusion with the component $\omega_{\hat{a} \hat{b} \hat{c}}$ connection, which will receive gravitino corrections. The reader is cautioned to not confuse the superspace connection $\Omega$ with the anholonomy coefficient $D_{\mathcal{A}} \mathcal{V}_{\mathcal{B}}{ }^{\mathcal{M}} \mathcal{V}_{\mathcal{M C}}$, which is frequently denoted $\Omega$ in the literature.

The connection $\Omega_{\mathcal{A B C}}$ has indices $\mathcal{B C}$ valued in $\mathcal{H}_{L} \times \mathrm{SO}(D-1,1)_{R}$. A conventional $\mathcal{H}_{L} \times \mathrm{SO}(D-1,1)_{R}$ connection should transform as

$$
\delta \Omega_{\mathcal{M A B}} \stackrel{?}{=} \mathbb{L}_{\xi} \Omega_{\mathcal{M A B}}+\partial_{\mathcal{M}} \lambda_{\mathcal{A B}}-\Omega_{\mathcal{M A}}{ }^{\mathcal{C}} \lambda_{\mathcal{C B}}+\Omega_{\mathcal{M B}}{ }^{\mathcal{C}} \lambda_{\mathcal{C A}}(-)^{a b}
$$

but we are going to find that this transformation rule is deformed by terms explicitly involving the DFT vielbein $\mathcal{V}_{\mathcal{M}}{ }^{\mathcal{A}}$. These deformations arise because we will be choosing the connections so that higher dimension torsion tensors vanish. Let us see what happens explicitly as we analyze the first few cases.

\footnotetext{
${ }^{8}$ In the case of heterotic DFT, Siegel identified $\mathcal{W}_{\bar{c}}{ }^{\beta}$ with the gauginos of the vector multiplets when $\bar{c}$ extended over the $n$ vector indices. In extending our approach to heterotic DFT, we would still keep $\mathcal{W}_{\bar{c}}{ }^{\beta}$ vanishing in all cases. The gaugino then would appear in the supervielbein as part of $\Psi_{\bar{a}}^{\beta}$, just as it does in component supersymmetric DFT [12]. The difference with Siegel's approach is that he includes an extra tangent space symmetry that allows one to remove the would-be gaugino from the supervielbein, just as with the dilatino.
} 
Define the torsion tensors so that

$$
\begin{aligned}
& \mathcal{T}_{\mathcal{A B C}}:=-3 \mathcal{D}_{[\mathcal{A}} \mathcal{V}_{\mathcal{B}}{ }^{\mathcal{M}} \mathcal{V}_{\mathcal{M C}]} \quad=\mathcal{F}_{\mathcal{A B C}}+3 \Omega_{[\mathcal{A B C}]}, \\
& \mathcal{T}_{\mathcal{A}}:=\mathcal{D}_{\mathcal{A}} \log \Phi+\mathcal{D}_{\mathcal{M}} \mathcal{V}_{\mathcal{A}}{ }^{\mathcal{M}}=\mathcal{F}_{\mathcal{A}}+\Omega^{\mathcal{B}}{ }_{\mathcal{B} \mathcal{A}} .
\end{aligned}
$$

At dimension $\frac{1}{2}$, there are four terms: $\mathcal{T}_{\alpha b c}, \mathcal{T}_{\alpha \bar{c}}, \mathcal{T}_{\alpha \beta}{ }^{\gamma}$, and $\mathcal{T}_{\alpha}$. Let's focus on the first two. It is easy to see from their definitions that they can be set to zero by appropriately choosing $\Omega_{\alpha b c}$ and $\Omega_{\alpha \overline{b c}}$,

$$
\mathcal{T}_{\alpha b c}:=\mathcal{F}_{\alpha b c}+\Omega_{\alpha b c} \equiv 0, \quad \mathcal{T}_{\alpha \overline{b c}}:=\mathcal{F}_{\alpha b c}+\Omega_{\alpha \overline{b c}} \equiv 0
$$

The second constraint is sensible in the sense that the variation of $\mathcal{F}_{\alpha \overline{b c}}$ implies a conventional transformation for $\Omega_{\alpha \overline{b c}}$. Explicitly,

$$
\begin{aligned}
& \delta \mathcal{F}_{\alpha \overline{b c}}=-D_{\alpha} \lambda_{\overline{b c}}+\lambda_{\alpha}{ }^{\beta} \mathcal{F}_{\beta \overline{b c}}-\lambda_{\bar{b}}{ }^{\bar{d}} \mathcal{F}_{\beta \overline{d c}}-\lambda_{\bar{c}} \bar{d}_{\beta \overline{b d}} \quad \Longrightarrow \\
& \delta \Omega_{\alpha \overline{b c}}=D_{\alpha} \lambda_{\overline{b c}}+\lambda_{\alpha}{ }^{\beta} \Omega_{\beta \overline{b c}}-\lambda_{\bar{b}}{ }^{\bar{d}} \Omega_{\alpha \overline{d c}}-\lambda_{\bar{c}}{ }^{\bar{d}} \Omega_{\alpha \overline{b d}} .
\end{aligned}
$$

However, the first constraint leads to an anomalous $\kappa$-dependent term

$$
\begin{aligned}
& \delta \mathcal{F}_{\alpha b c}=-D_{\alpha} \lambda_{b c}+\lambda_{\alpha}{ }^{\beta} \mathcal{F}_{\beta b c}+\lambda_{b}{ }^{d} \mathcal{F}_{\alpha d c}+\lambda_{c}{ }^{d} \mathcal{F}_{\alpha b d}+2 \mathcal{F}_{\alpha \gamma[b} \lambda_{c]}{ }^{\gamma} \\
& \delta \Omega_{\alpha b c}=D_{\alpha} \lambda_{b c}+\lambda_{\alpha}{ }^{\beta} \Omega_{\beta b c}+\lambda_{b}{ }^{d} \Omega_{\alpha d c}+\lambda_{c}{ }^{d} \Omega_{\alpha b d}-2 \kappa\left(\gamma_{[b}\right)_{\alpha \gamma} \lambda_{c]}{ }^{\gamma},
\end{aligned}
$$

where we have used the explicit form of $\mathcal{F}_{\alpha \gamma b}$.

There is a very simple reason for this. The natural $\mathcal{H}_{L}$ algebra action on covariant derivatives $\mathcal{D}_{\mathcal{A}}$ is $\delta_{\lambda} \mathcal{D}_{\mathcal{A}}=\lambda_{\mathcal{A}}{ }^{\mathcal{B}} \mathcal{D}_{\mathcal{B}}$. For the torsion tensor, this means that $\delta_{\lambda} \mathcal{T}_{\mathcal{A B C}}=$

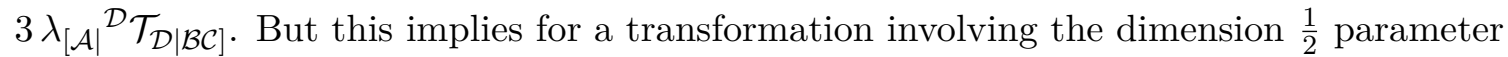
$\lambda_{a}{ }^{\beta}$, that $\mathcal{T}_{\alpha b c}$ should transform into the dimension zero $\mathcal{T}_{\alpha \beta c}$. The latter is non-vanishing. Requiring the former to vanish is inconsistent unless the $\lambda$ transformations are modified. We will see that (as for $\Omega_{\alpha b c}$ ), we can consistently modify the transformations of the $\Omega$ connections to accommodate this.

The other torsion tensors at dimension $\frac{1}{2}$ are

$$
\mathcal{T}_{\alpha \beta}{ }^{\gamma}=\mathcal{F}_{\alpha \beta}{ }^{\gamma}+2 \Omega_{(\alpha \beta)}{ }^{\gamma}, \quad \mathcal{T}_{\alpha}=\mathcal{F}_{\alpha}-\Omega_{\beta \alpha}{ }^{\beta}
$$

These involve a spin connection that has already been defined, since $\Omega_{\alpha \beta} \gamma=\frac{1}{4} \Omega_{\alpha b c}\left(\gamma^{b c}\right)_{\beta}{ }^{\gamma}$. Using the variation of $\Omega_{\alpha b c}$ defined above, the $\lambda$ transformation of $\mathcal{T}_{\alpha \beta} \gamma$, up to covariant terms involving $\lambda_{a b}$, becomes

$$
\Delta_{\lambda} \mathcal{T}_{\alpha \beta}{ }^{\gamma}=\kappa \delta_{(\alpha}^{\gamma}\left(\gamma^{c} \lambda_{c}\right)_{\beta)}-\frac{1}{2} \kappa\left(\gamma_{b}\right)_{\alpha \beta}\left(\gamma^{b} \gamma^{c} \lambda_{c}\right)^{\gamma}
$$

For convenience, we have suppressed indices for sequential spinor contractions, so that e.g. $\left(\gamma^{b} \gamma^{c} \lambda_{c}\right)^{\gamma}=\left(\gamma^{b}\right)^{\gamma \delta}\left(\gamma^{c}\right)_{\delta \epsilon} \lambda_{c}{ }^{\epsilon}$. This variation cancels out when $\lambda_{c}{ }^{\gamma}$ is $\gamma$-traceless. If this assumption is not made, then one must introduce a spin- $1 / 2$ field to populate $\mathcal{T}_{\alpha \beta}{ }^{\gamma}$. As we have already mentioned, this field would ultimately play the role of the dilatino. As we have elected to keep the dilatino within the supervielbein, we will take $\lambda_{c}{ }^{\alpha}$ to be purely 
spin-3/2 without further comment. It follows that $\Delta_{\lambda} \mathcal{T}_{\alpha \beta}{ }^{\gamma}$ vanishes. Similar comments pertain to $\mathcal{T}_{\alpha}$, whose variation is $\Delta_{\lambda} \mathcal{T}_{\alpha}=-\frac{9}{2} \kappa\left(\gamma^{c} \lambda_{c}\right)_{\alpha}$, which similarly vanishes.

Ultimately we will require both $\mathcal{T}_{\alpha \beta}{ }^{\gamma}$ and $\mathcal{T}_{\alpha}$ to vanish as a constraint on the geometry (as there is no covariant dimension $\frac{1}{2}$ quantity at the component level to identify them with), but for now, we will leave them unspecified. At this point we have defined all dimension $\frac{1}{2}$ components of $\Omega$.

Let's move on to dimension 1 torsions. There are four torsions that are familiar from bosonic DFT and we constrain them in the same way:

$$
\begin{array}{ll}
\mathcal{T}_{a b c}=\mathcal{F}_{a b c}+3 \Omega_{[a b c]} \equiv 0, & \mathcal{T}_{\overline{a b c}}=\mathcal{F}_{\overline{a b c}}+3 \Omega_{[\overline{a b c}]} \equiv 0, \\
\mathcal{T}_{\bar{a} b c}=\mathcal{F}_{\bar{a} b c}+\Omega_{\bar{a} b c} \equiv 0, & \mathcal{T}_{a \overline{b c}}=\mathcal{F}_{a \overline{b c}}+\Omega_{a \overline{b c}} \equiv 0
\end{array}
$$

As in the bosonic case, this defines only the totally antisymmetric parts of $\Omega_{a b c}$ and $\Omega_{\overline{a b c}}$. It implies the gauge transformations

$$
\begin{aligned}
\Delta_{\lambda} \Omega_{[a b c]} & =D_{[a} \lambda_{b c]}+\lambda_{[a}^{\gamma} \Omega_{\gamma b c]}, & \Delta_{\lambda} \Omega_{[\overline{a b c}]} & =D_{[\bar{a}} \lambda_{\overline{b c}]}, \\
\Delta_{\lambda} \Omega_{a \overline{b c}} & =D_{a} \lambda_{\overline{b c}}+\lambda_{a}^{\gamma} \Omega_{\gamma \overline{b c}}, & \Delta_{\lambda} \Omega_{\bar{a} b c} & =D_{\bar{a}} \lambda_{b c}
\end{aligned}
$$

where again we use $\Delta_{\lambda}$ to denote $\lambda$ transformations up to covariant $\lambda_{a b}$ or $\lambda_{\overline{a b}}$ terms.

Two more dimension 1 torsions involve only the connections $\Omega_{\bar{a} b c}$ and $\Omega_{\overline{a b c}}$ :

$$
\mathcal{T}_{\bar{a}}=\mathcal{F}_{\bar{a}}-\Omega_{\bar{b}}^{\overline{b a}} \equiv 0, \quad \mathcal{T}_{\bar{a} \beta}{ }^{\gamma}=\mathcal{F}_{\bar{a} \beta}{ }^{\gamma}+\Omega_{\bar{a} \beta}{ }^{\gamma}
$$

The first can be set to zero by fixing the trace of the $\mathrm{SO}(9,1)_{R}$ spin connection. The second torsion cannot be constrained, but we can check that its non-covariant $\lambda$ transformation vanishes, $\Delta_{\lambda} \mathcal{T}_{\bar{a} \beta} \gamma=0$. Again, this is consistent with it later being fixed to zero as a geometric constraint.

The remaining dimension 1 torsions both involve the additional $\mathcal{H}_{L}$ connection $\Omega_{\alpha b}{ }^{\gamma}$ :

$$
\mathcal{T}_{a}=\mathcal{F}_{a}+\Omega^{b}{ }_{b a}-\Omega_{\beta}{ }^{\beta}{ }_{a}, \quad \mathcal{T}_{a \beta}{ }^{\gamma}=\mathcal{F}_{a \beta}{ }^{\gamma}+\Omega_{a \beta}{ }^{\gamma}-\Omega_{\beta a}{ }^{\gamma} .
$$

The first torsion can be related to the second by defining

$$
\Omega^{b}{ }_{b a}:=-\mathcal{F}_{a}-\mathcal{F}_{a \beta}{ }^{\beta} \quad \Longrightarrow \quad \mathcal{T}_{a}=-\mathcal{T}_{a \beta}{ }^{\beta}
$$

The second torsion can be used to define $\Omega_{\alpha b}{ }^{\beta}$ if we suitably project onto the spin- $3 / 2$ part,

$$
\Omega_{\alpha b}^{\beta}:=\left(\mathcal{F}_{c \alpha}{ }^{\gamma}+\Omega_{c \alpha}{ }^{\gamma}\right)\left(\delta_{b}{ }^{c} \delta_{\gamma}^{\beta}-\frac{1}{10}\left(\gamma^{c} \gamma_{b}\right)_{\gamma}{ }^{\beta}\right) \quad \Longrightarrow \quad \mathcal{T}_{a \beta}{ }^{\gamma}=\frac{1}{10}\left(\gamma_{a}\right)^{\gamma \alpha} \mathcal{T}_{\alpha \beta}
$$

where $\mathcal{T}_{\alpha \beta}$ has no particular symmetry property for its two indices.

Something a little bit subtle is occurring above. We are using $\Omega_{c a b}$ to define $\Omega_{\alpha b}{ }^{\beta}$ even though not all of $\Omega_{c a b}$ is actually defined. Another way of saying this is that the definitions of $\Omega_{c a b}$ we have made allow us to assign it a transformation

$$
\left(\delta_{\Lambda}+\Delta_{\lambda}\right) \Omega_{a b c}=D_{a} \lambda_{b c}+\lambda_{a}^{\gamma} \Omega_{\gamma b c}-\frac{2}{9} \kappa \eta_{a[b}\left(\gamma_{c]}\right)_{\alpha \beta} \lambda^{\alpha \beta}+\Lambda_{a \mid b c}
$$


where $\Lambda_{a \mid b c}$ parametrizes the ambiguity in its definition. This same ambiguity now appears in $\Omega_{\beta a}{ }^{\gamma}$, which transforms as

$$
\begin{aligned}
\left(\delta_{\Lambda}+\Delta_{\lambda}\right) \Omega_{\beta a}{ }^{\gamma}= & D_{\beta} \lambda_{a}^{\gamma}+\Omega_{\beta}{ }^{b c}\left(\frac{1}{4} \lambda_{a}^{\alpha}\left(\gamma_{b c}\right)_{\alpha}^{\gamma}-\eta_{a b} \lambda_{c}^{\gamma}\right) \\
& +\kappa\left(\gamma_{a}\right)_{\beta \delta} \lambda^{\delta \gamma}-\frac{1}{18} \kappa\left(\gamma_{a b}\right)_{\beta}{ }^{\gamma}\left(\gamma^{b}\right)_{\delta \epsilon} \lambda^{\delta \epsilon}+\frac{1}{4}\left(\gamma^{b c}\right)_{\beta}{ }^{\gamma} \Lambda_{a \mid b c}
\end{aligned}
$$

All dimension 1 components of $\Omega$ are now defined up to the irreducible hook representation ambiguities $\Lambda_{a \mid b c}$ and $\Lambda_{\overline{a \mid b c}}$.

Next we turn to the dimension $\frac{3}{2}$ torsions:

$$
\begin{aligned}
\mathcal{T}_{\overline{a b}}{ }^{\alpha} & =\mathcal{F}_{\overline{a b}}{ }^{\alpha}+\Omega^{\alpha}{ }_{\overline{a b}}, \\
\mathcal{T}_{a \bar{b}}{ }^{\alpha} & =\mathcal{F}_{a \bar{b}}{ }^{\alpha}-\Omega_{\bar{b} a}{ }^{\alpha} \\
\mathcal{T}_{a b}{ }^{\alpha} & =\mathcal{F}_{a b}{ }^{\alpha}+\Omega^{\alpha}{ }_{a b}+2 \Omega_{[a b]}{ }^{\alpha} \\
\mathcal{T}_{\alpha}{ }^{\beta \gamma} & =\mathcal{F}_{\alpha}{ }^{\beta \gamma}+\Omega_{\alpha}{ }^{\beta \gamma}+\frac{1}{2}\left(\gamma^{b c}\right)_{\alpha}{ }^{(\beta} \Omega^{\gamma)}{ }_{b c}, \\
\mathcal{T}^{\alpha} & =\mathcal{F}^{\alpha}+\Omega^{b}{ }^{\alpha}+\frac{1}{4}\left(\gamma^{b c}\right)_{\beta}{ }^{\alpha} \Omega^{\beta}{ }_{b c}-\Omega_{\beta}{ }^{\beta \alpha}
\end{aligned}
$$

The first four equations lead to natural definitions for the $\Omega$ 's involved:

$$
\begin{aligned}
& \Omega^{\alpha} \overline{a b}:=-\mathcal{F}_{\overline{a b}}{ }^{\alpha} \quad \Longrightarrow \quad \mathcal{T}_{\overline{a b}}{ }^{\alpha} \equiv 0, \\
& \Omega_{\bar{b} a}{ }^{\alpha}:=-\mathcal{F}_{\bar{b} b}{ }^{\beta}\left(\delta_{a}{ }^{b} \delta_{\beta}{ }^{\alpha}-\frac{1}{10}\left(\gamma^{b} \gamma_{a}\right){ }^{\alpha}\right) \quad \Longrightarrow \quad \mathcal{T}_{a \bar{b}}{ }^{\alpha} \equiv \frac{1}{10}\left(\gamma_{a}\right)^{\alpha \beta} \mathcal{T}_{\beta \bar{b}}, \\
& \Omega^{\alpha}{ }_{a b}:=-\mathcal{F}_{a b}{ }^{\alpha}-2 \Omega_{[a b]}{ }^{\alpha} \quad \Longrightarrow \quad \mathcal{T}_{a b}{ }^{\alpha} \equiv 0, \\
& \Omega_{\alpha}{ }^{\beta \gamma}:=-\mathcal{F}_{\alpha}{ }^{\beta \gamma}-\frac{1}{2}\left(\gamma^{b c}\right)_{\alpha}{ }^{(\beta} \Omega^{\gamma)}{ }_{b c} \quad \Longrightarrow \mathcal{T}_{\alpha}{ }^{\beta \gamma} \equiv 0
\end{aligned}
$$

Remarkably, there is no constraint on $\Omega_{a b}{ }^{\gamma}$, which remains completely undetermined. These choices lead to the transformation rules

$$
\begin{aligned}
& \left(\delta_{\Lambda}+\Delta_{\lambda}\right) \Omega_{a b}^{\alpha}=D^{\alpha} \lambda_{a b}+\lambda^{\alpha \gamma} \Omega_{\gamma a b}+\lambda^{\alpha c} \Omega_{c a b}-2 \Lambda_{[a, b]}^{\alpha}, \\
& \left(\delta_{\Lambda}+\Delta_{\lambda}\right) \Omega_{a b}{ }^{\beta}=D_{a} \lambda_{b}{ }^{\beta}+\lambda_{a}^{\gamma} \Omega_{\gamma b}{ }^{\beta}+\Omega_{a}^{c d}\left(\frac{1}{4} \lambda_{b}^{\gamma}\left(\gamma_{c d}\right)_{\gamma}^{\beta}-\eta_{b c} \lambda_{d}^{\beta}\right)+\Lambda_{a, b}{ }^{\beta}, \\
& \left(\delta_{\Lambda}+\Delta_{\lambda}\right) \Omega_{\alpha}{ }^{\beta \gamma}=D_{\alpha} \lambda^{\beta \gamma}+\frac{1}{2} \Omega_{\alpha b c} \lambda^{\delta(\beta}\left(\gamma^{b c}\right)_{\delta}^{\gamma)}+2 \Omega_{\alpha c}{ }^{(\gamma} \lambda^{c \beta)}+\left(\gamma^{b c}\right)_{\alpha}{ }^{(\beta} \Lambda_{b, c}{ }^{\gamma)}
\end{aligned}
$$

where $\Lambda_{a, b}{ }^{\beta}$ is $\gamma$-traceless in the last two indices. This parametrizes the total ambiguity in the definition of $\Omega_{a b}{ }^{\beta}$. While it could be used to absorb all the other terms in $\delta \Omega_{a b}{ }^{\beta}$, we have chosen to define $\delta \Omega_{a b}{ }^{\beta}$ to match the patterns that have been exhibited so far.

The remaining torsions at dimension 2 and dimension $\frac{5}{2}$ are

$$
\mathcal{T}_{a}^{\beta \gamma}=\mathcal{F}_{a}{ }^{\beta \gamma}+\Omega_{a}{ }^{\beta \gamma}-2 \Omega^{(\beta}{ }_{a}^{\gamma)}, \quad \mathcal{T}_{\bar{a}}^{\beta \gamma}=\mathcal{F}_{\bar{a}}{ }^{\beta \gamma}+\Omega_{\bar{a}^{\beta \gamma}}{ }^{\beta}, \quad \mathcal{T}^{\alpha \beta \gamma}=\mathcal{F}^{\alpha \beta \gamma}+3 \Omega^{(\alpha \beta \gamma)} .
$$

These suggest the definitions

$$
\begin{aligned}
\Omega_{a}{ }^{\beta \gamma} & :=-\mathcal{F}_{a}{ }^{\beta \gamma}+2 \Omega^{\left({ }_{a}{ }_{a} \gamma\right)} & \Longrightarrow \mathcal{T}_{a}{ }^{\beta \gamma} & \equiv 0, \\
\Omega_{\bar{a}}{ }^{\beta \gamma} & :=-\mathcal{F}_{\bar{a}}{ }^{\beta \gamma} & \Longrightarrow \mathcal{T}_{\bar{a}}{ }^{\beta \gamma} & \equiv 0, \\
\Omega^{(\alpha \beta \gamma)}: & :=-\frac{1}{3} \mathcal{F}^{\alpha \beta \gamma} & \Longrightarrow \mathcal{T}^{\alpha \beta \gamma} & \equiv 0 .
\end{aligned}
$$


The last definition leaves the (irreducible) hook representation in $\Omega^{\alpha \beta \gamma}$ unfixed. These results lead to the transformation rules

$$
\begin{aligned}
& \left(\delta_{\Lambda}+\Delta_{\lambda}\right) \Omega_{\bar{a}}{ }^{\beta \gamma}=D_{\bar{a}} \lambda^{\beta \gamma}+\frac{1}{2} \Omega_{\bar{a} b c} \lambda^{\delta(\beta}\left(\gamma^{b c}\right)_{\delta}^{\gamma)}+2 \Omega_{\bar{a} c}{ }^{(\gamma} \lambda^{c \beta)} \\
& \left(\delta_{\Lambda}+\Delta_{\lambda}\right) \Omega_{a}{ }^{\beta \gamma}=D_{a} \lambda^{\beta \gamma}+\lambda_{a}^{\alpha} \Omega_{\alpha}{ }^{\beta \gamma}+\frac{1}{2} \Omega_{a b c} \lambda^{\delta(\beta}\left(\gamma^{b c}\right)_{\delta}{ }^{\gamma)}+2 \Omega_{a c}{ }^{(\gamma} \lambda^{c \beta)}+2 \Lambda^{(\beta}{ }_{, b}^{\gamma)}, \\
& \left(\delta_{\Lambda}+\Delta_{\lambda}\right) \Omega^{\alpha \beta \gamma}=D^{\alpha} \lambda^{\beta \gamma}+\lambda^{\alpha \mathcal{D}} \Omega_{\mathcal{D}}{ }^{\beta \gamma}+\frac{1}{2} \Omega^{\alpha}{ }_{b c} \lambda^{\delta(\beta}\left(\gamma^{b c}\right)_{\delta}{ }^{\gamma)}+2 \Omega^{\alpha}{ }_{c}{ }^{(\gamma} \lambda^{c \beta)}+\Lambda^{\alpha \mid \beta \gamma} \\
& \left(\delta_{\Lambda}+\Delta_{\lambda}\right) \Omega^{\alpha}{ }_{b}^{\gamma}=D^{\alpha} \lambda_{b}{ }^{\gamma}+\lambda^{\alpha \mathcal{D}} \Omega_{\mathcal{D} b}{ }^{\gamma}+\Omega^{\alpha c d}\left(\frac{1}{4} \lambda_{b}{ }^{\beta}\left(\gamma_{c d}\right)_{\beta}{ }^{\gamma}-\eta_{b c} \lambda_{d}{ }^{\gamma}\right)+\Lambda^{\alpha}{ }_{, b}^{\gamma}
\end{aligned}
$$

where the hook representation $\Lambda^{\alpha \mid \beta \gamma}\left(\right.$ with $\left.\Lambda^{(\alpha \mid \beta \gamma)}=0\right)$ parametrizes the ambiguity in $\Omega^{\alpha \beta \gamma}$.

This completes our analysis of the tangent space $\Omega$ connections. Up to the ambiguities parametrized by the $\Lambda$ parameters, we have determined each of these connections. Their transformation rules can now be summarized in a uniform way, where we take $\Omega_{\mathcal{M A B}}:=$ $\mathcal{V}_{\mathcal{M}}{ }^{\mathcal{C}} \Omega_{\mathcal{C A B}}$

$$
\begin{aligned}
\left(\delta_{\Lambda}+\Delta_{\lambda}\right) \Omega_{\mathcal{M} \overline{b c}}= & \partial_{\mathcal{M}} \lambda_{\overline{b c}}+\mathcal{V}_{\mathcal{M}}{ }^{\bar{a}} \Lambda_{\bar{a} \mid \overline{b c}}, \\
\left(\delta_{\Lambda}+\Delta_{\lambda}\right) \Omega_{\mathcal{M} b c}= & \partial_{\mathcal{M}} \lambda_{b c}-2 \kappa \mathcal{V}_{\mathcal{M}}{ }^{\alpha}\left(\gamma_{[b}\right)_{\alpha \gamma} \lambda_{c]}{ }^{\gamma}+\mathcal{V}_{\mathcal{M}}{ }^{a}\left(-\frac{2}{9} \kappa \eta_{a[b}\left(\gamma_{c]}\right)_{\beta \gamma} \lambda^{\beta \gamma}+\Lambda_{a \mid b c}\right) \\
& -2 \mathcal{V}_{\mathcal{M} \alpha} \Lambda_{[b, c]}{ }^{\alpha} \\
\left(\delta_{\Lambda}+\Delta_{\lambda}\right) \Omega_{\mathcal{M} b}{ }^{\gamma}= & \partial_{\mathcal{M}} \lambda_{b}{ }^{\gamma}+\Omega_{\mathcal{M}}{ }^{c d}\left(\frac{1}{4} \lambda_{b}{ }^{\alpha}\left(\gamma_{c d}\right)_{\alpha}{ }^{\gamma}-\eta_{b c} \lambda_{d}{ }^{\gamma}\right) \\
& +\mathcal{V}_{\mathcal{M}}{ }^{\alpha}\left(\kappa\left(\gamma_{b}\right)_{\alpha \delta} \lambda^{\delta \gamma}-\frac{1}{18} \kappa\left(\gamma_{b c}\right)_{\alpha}{ }^{\gamma}\left(\gamma^{c}\right)_{\delta \epsilon} \lambda^{\delta \epsilon}+\frac{1}{4}\left(\gamma^{c d}\right)_{\alpha}{ }^{\gamma} \Lambda_{b \mid c d}\right) \\
& +\mathcal{V}_{\mathcal{M}}{ }^{a} \Lambda_{a, b}{ }^{\gamma}+\mathcal{V}_{\mathcal{M} \alpha} \Lambda_{, b}^{\alpha}{ }^{\gamma}, \\
\left(\delta_{\Lambda}+\Delta_{\lambda}\right) \Omega_{\mathcal{M}}^{\beta \gamma}= & \partial_{\mathcal{M}} \lambda^{\beta \gamma}+\frac{1}{2} \Omega_{\mathcal{M} b c} \lambda^{\delta(\beta}\left(\gamma^{b c}\right)_{\delta}{ }^{\gamma)}+2 \Omega_{\mathcal{M} c}{ }^{(\gamma} \lambda^{c \beta)} \\
& +\mathcal{V}_{\mathcal{M}}{ }^{\alpha}\left(\gamma^{b c}\right)_{\alpha}{ }^{(\beta} \Lambda_{b, c}{ }^{\gamma)}+2 \mathcal{V}_{\mathcal{M}}{ }^{a} \Lambda^{(\beta,}{ }_{, a}{ }^{\gamma)}+\mathcal{V}_{\mathcal{M} \alpha} \Lambda^{\alpha \mid \beta \gamma}
\end{aligned}
$$

The key features of these transformations are as follows. The terms involving $\lambda$ that are $\kappa$-independent are nothing more than the expected $\operatorname{OSp}(9,1 \mid 32)$ transformations given in (4.7). These include the inhomogeneous $\partial_{\mathcal{M}} \lambda$ terms and the terms where $\Omega$ is transformed into another $\Omega$. The remaining terms, which are $\kappa$-dependent or involve the additional $\Lambda$ terms, correspond to rotations of $\Omega$ into the supervielbein. This look like shift symmetries when $\Omega_{\mathcal{M}}$ is written with a tangent space index.

At this point, there seems no reason to take the $\Lambda$ parameters seriously as local transformations. So far they are only parametrizing our ignorance of the $\Omega$ connections. But we will now check that the $\Lambda$ transformations are actually required for closure of the gauge transformations. To condense the notation, we will employ BRST terminology, and give the algebra of gauge transformations with the nilpotent BRST operator $\delta$ acting on (graded) anti-commuting parameters $\lambda$, so that, for $\left[\delta_{1}, \delta_{2}\right]=\delta_{12}$, the closure of $\mathrm{SO}(9,1)_{L}$ transformations

$$
\lambda_{12 a b}=-\lambda_{1 a}{ }^{c} \lambda_{2 c b}+\lambda_{2 a}{ }^{c} \lambda_{1 c b}
$$

becomes a more compact expression

$$
\delta \lambda_{a b}=-\lambda_{a}^{c} \lambda_{c b}
$$


The other tangent space algebraic relations are encoded in

$$
\delta \lambda_{a}{ }^{\beta}=-\lambda_{a}{ }^{c} \lambda_{c}{ }^{\beta}-\lambda_{a}{ }^{\gamma} \lambda_{\gamma}{ }^{\beta}, \quad \delta \lambda^{\alpha \beta}=\lambda^{b \alpha} \lambda_{b}{ }^{\beta}-2 \lambda^{\gamma(\alpha} \lambda_{\gamma}{ }^{\beta)} .
$$

These expressions are just the explicit decomposition of $\delta \lambda_{\mathcal{A B}}=-\lambda_{\mathcal{A}}{ }^{\mathcal{C}} \lambda_{\mathcal{C B}}$. More interesting are the closure conditions on the additional $\Lambda$ symmetries. For example, we find for $\Lambda_{a \mid b c}$ that

$$
\delta \Lambda_{a \mid b c}=2 \kappa \lambda_{a}{ }^{\alpha}\left(\gamma_{[b}\right)_{\alpha \beta} \lambda_{c]}{ }^{\beta}+\frac{2}{9} \kappa \eta_{a[b}\left(\gamma_{c]}\right)_{\alpha \beta} \lambda^{d \alpha} \lambda_{d}{ }^{\beta}-\lambda_{a}{ }^{d} \Lambda_{d \mid b c}-\lambda_{b}{ }^{d} \Lambda_{a \mid d c}-\lambda_{c}{ }^{d} \Lambda_{a \mid b d} .
$$

This enforces that the closure of $\lambda_{a}{ }^{\alpha}$ transformations requires a $\Lambda_{a \mid b c}$ transformation. Similarly, for the other $\Lambda$ parameters we find (writing $\Delta$ to suppress the obvious $\lambda_{a}^{b}$ contributions to these terms)

$$
\begin{aligned}
\Delta \Lambda_{b a}{ }^{\beta}= & \kappa\left(\gamma_{a}\right)_{\alpha \gamma} \lambda^{\beta \alpha} \lambda_{b}{ }^{\gamma}-\frac{1}{9} \kappa\left(\gamma_{a}\right)_{\alpha \gamma} \lambda^{\alpha \gamma} \lambda_{b}{ }^{\beta}+\frac{1}{9} \kappa \eta_{b a}\left(\gamma_{c}\right)_{\alpha \gamma} \lambda^{\alpha \gamma} \lambda_{c}{ }^{\beta} \\
& -\frac{1}{18} \kappa\left(\gamma^{c}\right)_{\alpha \gamma}\left(\gamma_{b c}\right)_{\delta}{ }^{\beta} \lambda^{\alpha \gamma} \lambda_{a}{ }^{\delta}-\frac{1}{18} \kappa\left(\gamma^{c}\right)_{\alpha \gamma}\left(\gamma_{a c}\right)_{\delta}{ }^{\beta} \lambda^{\alpha \gamma} \lambda_{b}{ }^{\beta} \\
& +\frac{1}{4} \Lambda_{b \mid c d}\left(\gamma^{c d}\right)_{\alpha}{ }^{\beta} \lambda_{a}{ }^{\alpha}+\frac{1}{4} \Lambda_{a \mid c d}\left(\gamma^{c d}\right)_{\alpha}{ }^{\beta} \lambda_{b}{ }^{\alpha}-\Lambda_{b \mid a c} \lambda^{c \beta} \\
\Delta \Lambda^{\alpha}{ }_{a}{ }^{\beta}= & -\kappa \lambda^{\alpha \gamma}\left(\gamma_{a}\right)_{\gamma \delta} \lambda^{\delta \beta}+\frac{1}{18} \kappa \lambda^{\alpha \epsilon}\left(\gamma_{a b}\right)_{\epsilon}{ }^{\beta}\left(\gamma^{b}\right)_{\gamma \delta} \lambda^{\gamma \delta} \\
& +\Lambda_{b a}{ }^{\beta} \lambda^{b \alpha}+\Lambda_{a b}{ }^{\alpha} \lambda^{b \beta}-\Lambda_{b a}{ }^{\alpha} \lambda^{b \beta}-\frac{1}{2} \Lambda_{b c}{ }^{\alpha} \lambda_{a}{ }^{\gamma}\left(\gamma^{b c}\right)_{\gamma}{ }^{\beta} \\
& +\frac{1}{4} \Lambda_{a \mid b c} \lambda^{\alpha \gamma}\left(\gamma^{b c}\right)_{\gamma}^{\beta} \\
\Delta \Lambda^{\gamma \mid \alpha \beta}= & -\Lambda_{a b}{ }^{\gamma}\left(\gamma^{a b}\right)_{\delta}{ }^{(\alpha} \lambda^{\beta) \delta}+\Lambda_{a b}{ }^{(\alpha}\left(\gamma^{a b}\right)_{\delta}^{\beta)} \lambda^{\gamma \delta}+2 \Lambda^{\gamma}{ }_{a}{ }^{(\alpha} \lambda^{a \beta)}-2 \Lambda^{\left(\alpha{ }_{a}{ }^{\beta)} \lambda^{a \gamma}\right.} .
\end{aligned}
$$

The point is that each of the additional $\Lambda$ transformations becomes necessary for closure when $\kappa \neq 0$. Moreover, we find no additional symmetry constraints on these parameters aside from $\gamma$-tracelessness of $\Lambda_{a b}{ }^{\beta}$ and $\Lambda_{b}^{\alpha}$ on their $b \beta$ indices, and the vanishing of the totally symmetric part of $\Lambda^{\alpha \mid \beta \gamma}$.

We will need to say a bit more about the structure of these additional transformations, but for now let us return to comment on the torsions that have been set to zero. These are summarized in table 2 . In the middle column, we have listed all the constraints that are purely conventional, meaning they arise from a specific choice of the $\Omega$ connection. The torsions in the right column remain. Some of these have physical constraints imposed to eliminate covariant objects in the torsion. These are true constraints on the supervielbein, as opposed to conventional choices of $\Omega$.

The structure of the unfixed torsions can be understood by considering the linearized supervielbein. Writing $\mathcal{V}_{\mathcal{M}}{ }^{\mathcal{A}}=\delta_{\mathcal{M}}{ }^{\mathcal{A}}+v_{\mathcal{M B}} \eta^{\mathcal{B} \mathcal{A}}(-1)^{b}$ for $v_{\mathcal{B} \mathcal{A}}$ a graded antisymmetric quantity, we can see that $v$ transforms as $\delta v_{\mathcal{B} \mathcal{A}}=2 \partial_{[\mathcal{B}} \xi_{\mathcal{A}]}-\lambda_{\mathcal{B} \mathcal{A}}$ under linearized diffeomorphisms and tangent space transformations. Using $\lambda$ as much as possible to fix $v$, we find the following surviving components. First are the negative dimension fields, $v_{\beta \alpha}, v_{\beta a}$, and $v_{\beta \bar{a}}$, and the zero-dimension field $v_{\alpha}{ }^{\beta}$. These transform as

$$
\begin{aligned}
& \delta v_{\beta \alpha}=-2 \partial_{(\beta} \xi_{\alpha)}, \quad \delta v_{\beta a}=\partial_{\beta} \xi_{a}+\partial_{a} \xi_{\beta}, \quad \delta v_{\beta \bar{a}}=-\partial_{\beta} \xi_{\bar{a}}+\partial_{\bar{a}} \xi_{\beta}, \\
& \delta v_{\beta}{ }^{\alpha}=\partial_{\beta} \xi^{\alpha}-\partial^{\alpha} \xi_{\beta}-\frac{1}{2}\left(\gamma^{c d}\right)_{\beta}{ }^{\alpha} \partial_{c} \xi_{d} .
\end{aligned}
$$

The remaining fields are analogous to what we find at the component level. There is the zero-dimension bosonic DFT vielbein $v_{\bar{b} a}$, the gravitino $v_{\bar{b}}{ }^{\alpha} \equiv \Psi_{\bar{b}}{ }^{\alpha}$, and the dilatino 


\begin{tabular}{c|c|c}
\hline dimension & conventional constraints & remaining torsions \\
\hline$-\frac{1}{2}$ & - & $\mathcal{T}_{\alpha \beta \gamma}=0$ \\
\hline 0 & - & $\mathcal{T}_{\alpha \beta c}=\kappa\left(\gamma_{c}\right)_{\alpha \beta} \quad \mathcal{T}_{\alpha \beta \bar{c}}=0$ \\
\hline$\frac{1}{2}$ & $\mathcal{T}_{\alpha b c}=\mathcal{T}_{\alpha \overline{b c}}=0$ & $\mathcal{T}_{\alpha b \bar{c}}=0$ \\
& & $\mathcal{T}_{\alpha \beta}{ }^{\gamma}, \mathcal{T}_{\alpha}$ \\
\hline 1 & $\mathcal{T}_{\hat{a} \hat{b} \hat{c}}=\mathcal{T}_{\hat{a}}=0$ & $\mathcal{T}_{\bar{a} \beta}{ }^{\gamma}$ \\
& $\mathcal{T}_{a \beta}{ }^{\gamma}=\frac{1}{10}\left(\gamma_{a}\right)^{\gamma \alpha} \mathcal{T}_{\alpha \beta}$ & \\
\hline$\frac{3}{2}$ & $\mathcal{T}_{\overline{a b}}{ }^{\alpha}=\mathcal{T}_{a b}{ }^{\alpha}=\mathcal{T}_{\alpha}{ }^{\beta \gamma}=0$ & \\
\hline 2 & $\mathcal{T}_{a \bar{b}}{ }^{\alpha}=\frac{1}{10}\left(\gamma_{a}\right)^{\gamma \alpha} \mathcal{T}_{\alpha \bar{b}}$ & - \\
\hline$\frac{5}{2}$ & $\mathcal{T}_{\hat{a}}^{\beta \gamma}=0$ & - \\
\hline
\end{tabular}

Table 2. Conventional and physical constraints on torsion. Conventional constraints arise from a specific choice of $\Omega_{\mathcal{A B C}}$. Some of the remaining torsions have additional physical constraints imposed.

$v_{b}^{\alpha} \equiv \frac{1}{10}\left(\gamma_{b}\right)^{\alpha \beta} \chi_{\beta}$. Their transformation rules are ${ }^{9}$

$$
\delta v_{\bar{b} a}=\partial_{\bar{b}} \xi_{a}+\partial_{a} \xi_{\bar{b}}, \quad \delta \Psi_{\bar{b}}^{\alpha}=\partial_{\bar{b}} \xi^{\alpha}+\partial^{\alpha} \xi_{\bar{b}}, \quad \delta \chi_{\alpha}=\left(\gamma^{b}\right)_{\alpha \beta}\left(\partial_{b} \xi^{\beta}-\partial^{\beta} \xi_{b}\right) .
$$

Finally, there is the linearized superdilaton $\Phi=1+\varphi$, transforming as

$$
\delta \varphi=\partial_{a} \xi^{a}+\partial_{\bar{a}} \xi^{\bar{a}}-\partial_{\alpha} \xi^{\alpha}-\partial^{\alpha} \xi_{\alpha} .
$$

Now we can consider the possible one derivative invariants one can build out of the linearized vielbein and the dilaton. These are in direct correspondence with the torsion tensors already identified. The ones with negative or vanishing dimension are

$$
\mathcal{T}_{\alpha \beta \gamma}=3 \partial_{(\alpha} v_{\beta \gamma)}, \quad \mathcal{T}_{\alpha \beta \hat{c}}=2 \partial_{(\alpha} v_{\beta) \hat{c}}+\partial_{\hat{c}} v_{\alpha \beta}, \quad \mathcal{T}_{\alpha b \bar{c}}=\partial_{\alpha} v_{b \bar{c}}-\partial_{b} v_{\alpha \bar{c}}+\partial_{\bar{c}} v_{\alpha b}
$$

These we have already constrained in (4.6). The ones at dimension $\frac{1}{2}$ are

$$
\begin{aligned}
\mathcal{T}_{\alpha \beta}{ }^{\gamma} & =2 \partial_{(\alpha} v_{\beta)}^{\gamma}+\partial^{\gamma} v_{\alpha \beta}+\partial_{c} v_{(\alpha d}\left(\gamma^{c d}\right)_{\beta)}^{\gamma}, \\
\mathcal{T}_{\alpha} & =\partial_{\alpha} \varphi-\partial^{b} v_{\alpha b}+\partial^{\bar{b}} v_{\alpha \bar{b}}+\partial_{\alpha} v_{\beta}^{\beta}+2 \partial^{\beta} v_{\alpha \beta} .
\end{aligned}
$$

These must vanish as well, as there are no covariant physical fields in supersymmetric DFT at this dimension, and we will impose their vanishing in due course. At dimension 1, we find

$$
\begin{aligned}
\mathcal{T}_{\bar{a} \beta}{ }^{\gamma}= & \partial_{\bar{a}} v_{\beta}^{\gamma}+\partial^{\gamma} v_{\beta \bar{a}}-\partial_{\beta} \Psi_{\bar{a}}^{\gamma}+\frac{1}{2}\left(\gamma^{c d}\right)_{\beta}^{\gamma} \partial_{c} v_{\bar{a} d}, \\
\mathcal{T}_{\alpha \beta} \equiv\left(\gamma^{b}\right)_{\alpha \gamma} \mathcal{T}_{b \beta}{ }^{\gamma}= & -\partial_{\beta} \chi_{\alpha}+\left(\gamma^{a}\right)_{\alpha \beta}\left(\frac{1}{2} \partial_{a} v_{\gamma}^{\gamma}+\frac{1}{2} \partial_{a} \varphi+\partial^{\gamma} v_{\gamma a}-\frac{1}{2} \partial^{\bar{b}} v_{\bar{b} a}\right) \\
& +\left(\gamma^{a}\right)_{\alpha \gamma}\left(\partial^{\gamma} v_{\beta a}+\partial_{a} v_{\beta}^{\gamma}\right)
\end{aligned}
$$

\footnotetext{
${ }^{9}$ When reducing to components in section 5 , we will solve the fermionic pieces of the section condition by choosing $\partial^{\alpha}=0$ to eliminate $\tilde{\theta}$ dependence. Then the linearized gravitino and the dilatino acquire their usual transformations. The physical linearized dilaton will presumably be the combination $\varphi+v_{\alpha}{ }^{\alpha}$. The other low dimension fields in (4.35) evidently can be set to zero using a Wess-Zumino gauge transformation - that is, by using the $\theta$-dependent parts of the diffeomorphisms to eliminate them.
} 
These should vanish as well. Finally at dimension $\frac{3}{2}$, we encounter

$$
\begin{aligned}
\mathcal{T}^{\alpha} & =\left(\gamma^{a}\right)^{\alpha \beta} \partial_{a} \chi_{\beta}-\partial^{\bar{b}} \Psi_{\bar{b}}{ }^{\alpha}+\partial^{\alpha} \varphi+2 \partial^{\beta} v_{\beta}{ }^{\alpha}+\partial^{\alpha} v_{\beta}{ }^{\beta}, \\
\mathcal{T}_{\alpha \bar{b}} \equiv\left(\gamma^{a}\right)_{\alpha \gamma} \mathcal{T}_{a \bar{b}}{ }^{\gamma} & =\left(\gamma^{a}\right)_{\alpha \beta} \partial_{a} \Psi_{\bar{b}}{ }^{\beta}-\partial_{\bar{b}} \chi_{\alpha}-\left(\gamma^{a}\right)_{\alpha \beta} \partial^{\beta} v_{\bar{b} a} .
\end{aligned}
$$

These correspond respectively to the linearized dilatino and gravitino equations of motion. As the superspace geometry places DFT on-shell, these should vanish, as we will show.

\subsection{A detour on tangent space symmetries and an extended algebra}

Before moving on to analyze the torsion constraints and Bianchi identities in superspace, we need to make a digression into the structure of the tangent space symmetries. As we have discussed, the closure of the orthosymplectic tangent space symmetries implies new local shift symmetries on the spin connection. But this conclusion was motivated purely by requiring consistency of the constraints that we seek to impose. In this subsection, we instead give a more abstract characterization of the algebra itself, motivated purely by closure of the Jacobi identity. Subsequently, we will show that the gauging of this algebra leads to the same transformations on $\Omega$, and implies the existence of higher connections.

Let us recall the normalization of the $\operatorname{OSp}(9,1 \mid 32)_{L}$ generators $M_{A B}$ so that

$$
\frac{1}{2}\left[\lambda^{B C} M_{C B}, P_{A}\right]=\lambda_{A}{ }^{B} P_{B}
$$

where $P_{A}=\left(P_{a}, Q_{\alpha}, \tilde{Q}^{\alpha}\right)$ are the supertranslation generators and the commutator should be understood as a graded commutator. The above is the conventional orthosymplectic action, but soon we will see it is deformed in various ways. To begin with, the only generators we are keeping are $M_{a b}$ (which includes a piece of $\left(\gamma^{a b}\right)_{\beta}{ }^{\alpha} M_{\alpha}{ }^{\beta}$ to rotate fermionic indices), $M_{\alpha b}$ (now restricted to be $\gamma$-traceless), and $M_{\alpha \beta}$. We use the above formula merely to fix normalizations of these operators.

The Lorentz generator acts as usual as

$$
\begin{aligned}
{\left[M_{c b}, P_{a}\right] } & =\eta_{b a} P_{c}-\eta_{c a} P_{b}, \\
{\left[M_{c b}, Q_{\alpha}\right] } & =-\frac{1}{2}\left(\gamma_{c b}\right)_{\alpha}{ }^{\beta} Q_{\beta}, \\
{\left[M_{c b}, \tilde{Q}^{\alpha}\right] } & =-\frac{1}{2}\left(\gamma_{c b}\right)^{\alpha}{ }_{\beta} \tilde{Q}^{\beta} .
\end{aligned}
$$

The generators $P_{A}$ themselves obey the algebra

$$
\left\{Q_{\alpha}, Q_{\beta}\right\}=-\kappa\left(\gamma^{c}\right)_{\alpha \beta} P_{c}, \quad\left[Q_{\alpha}, P_{b}\right]=-\kappa\left(\gamma_{b}\right)_{\alpha \gamma} \tilde{Q}^{\gamma} .
$$

As usual for supersymmetry, this describes a rigid (but not flat) supergeometry with a constant fixed torsion tensor. The presence of this torsion tensor has deep consequences for the algebra, which we are about to discover!

The tangent space generator at dimension $-\frac{1}{2}$ is the $\gamma$-traceless fermionic operator $M_{\alpha a}$. Its algebra with $P_{A}$ turns out to be

$$
\begin{aligned}
\left\{M_{\beta b}, Q_{\alpha}\right\} & =\kappa\left(\gamma^{c}\right)_{\beta \alpha} M_{b c}-\frac{1}{10} \kappa\left(\gamma_{b} \gamma^{c d}\right)_{\beta \alpha} M_{c d}, \\
{\left[M_{\beta b}, P_{a}\right] } & =\eta_{b a} Q_{\beta}-\frac{1}{10}\left(\gamma_{b} \gamma_{a}\right)_{\beta}^{\gamma} Q_{\gamma} \\
\left\{M_{\beta b}, \tilde{Q}^{\alpha}\right\} & =-\delta_{\beta}{ }^{\alpha} P_{b}+\frac{1}{10}\left(\gamma_{b} \gamma^{c}\right)_{\beta}{ }^{\alpha} P_{c},
\end{aligned}
$$


where in each expression the second term is required to project onto spin-3/2. The second and third algebraic relations follow directly from (4.42). The first relation would be zero if we employed (4.42). That this cannot be zero follows from the Jacobi identity $\left[\left\{M_{\alpha a}, Q_{\beta}\right\}, Q_{\gamma}\right]+\cdots$ in the presence of the torsion term (4.44). One must make a $\kappa$ dependent deformation and the expression (4.45a) does the job. Similarly, at dimension $-1, M_{\alpha \beta}$ acts as

$$
\begin{aligned}
{\left[M_{\gamma \beta}, Q_{\alpha}\right] } & =-2 \kappa\left(\gamma^{b}\right)_{\alpha(\beta} M_{\gamma) b}+\frac{1}{9} \kappa\left(\gamma^{b}\right)_{\gamma \beta} M_{\alpha b}, \\
{\left[M_{\gamma \beta}, P_{a}\right] } & =\frac{2}{9} \kappa\left(\gamma^{b}\right)_{\gamma \beta} M_{b a} \\
{\left[M_{\gamma \beta}, \tilde{Q}^{\alpha}\right] } & =2 \delta^{\alpha}{ }_{(\gamma} Q_{\beta)} .
\end{aligned}
$$

Now the first and second relations involve deformations and are required by closure of the Jacobi identity.

These results dovetail with the explicit connection transformations. The three generators $P_{A}=\left(Q_{\alpha}, P_{a}, \tilde{Q}^{\alpha}\right)$ are represented on superfields by covariant derivatives $\nabla_{A}=\mathcal{V}_{A} \mathcal{M}_{\mathcal{M}_{\mathcal{M}}-}$ $\frac{1}{2} \Omega_{A}{ }^{B C} M_{C B}+\cdots$. The algebra $\left[M_{C B}, P_{A}\right]$ is then represented via $\delta_{\lambda} \nabla_{A}$. Computing $\delta_{\lambda}$ using (4.29) leads to the (4.45) and (4.46).

But we are not finished. Closure of the $M_{A B}$ algebra on the connections required a new local symmetry, and this should appear at the algebraic level. This ought to be a new generator at dimension -1 in the irreducible hook representation, which we denote $K_{a \mid b c}$. Working backwards from the $\Lambda$ transformations on $\Omega$, we conclude that

$$
\begin{array}{ll}
{\left[K_{a \mid b c}, P_{d}\right]=\left.\frac{1}{2} \eta_{d a} M_{b c}\right|_{\text {proj }}} & =\frac{1}{3} \eta_{d a} M_{b c}-\frac{1}{3} \eta_{d[b} M_{c] a}+\frac{1}{9} \eta_{a[b} M_{c] d}, \\
{\left[K_{a \mid b c}, Q_{\alpha}\right]=-\left.\frac{1}{4}\left(\gamma_{b c}\right)_{\alpha}{ }^{\beta} M_{\beta a}\right|_{\text {proj }}} & =-\frac{1}{4}\left(\gamma_{b c}\right)_{\alpha}{ }^{\beta} M_{\beta a}+\frac{1}{4}\left(\gamma_{[b c}\right)_{\alpha}{ }^{\beta} M_{\beta a]}+\frac{1}{18} \eta_{a[b} M_{\alpha c]}, \\
{\left[K_{a \mid b c}, \tilde{Q}^{\alpha}\right]} & =0
\end{array}
$$

where we introduce the notation $\left.\right|_{\text {proj }}$ to denote projection onto the appropriate representation (in this case, the irreducible hoop representation $a \mid b c$ ) implied by the left-hand side of the equation. To verify the necessity of this generator at the algebraic level, consider the anticommutator of $M_{\alpha a}$ with itself. This is given by

$$
\left\{M_{\alpha a}, M_{\beta b}\right\}=-\left.\eta_{a b} M_{\alpha \beta}\right|_{\text {proj }}+\kappa \text {-dependent terms }
$$

The leading term is expected from the orthosymplectic algebra. That $\kappa$-dependent terms are required follows by considering further commutation with $Q_{\alpha}$. The result is

$$
\begin{aligned}
\left\{M_{\alpha a}, M_{\beta b}\right\}= & -\eta_{a b} M_{\alpha \beta}+\left.2 \kappa\left(\gamma^{c}\right)_{\beta \alpha}\left(K_{b \mid a c}+K_{a \mid b c}\right)\right|_{\text {proj }} \\
= & -\eta_{a b} M_{\alpha \beta}+\frac{1}{10}\left(\gamma_{a} \gamma_{b}\right)_{\alpha}{ }^{\delta} M_{\delta \beta}+\frac{1}{10}\left(\gamma_{b} \gamma_{a}\right)_{\beta}{ }^{\delta} M_{\alpha \delta}-\frac{1}{100}\left(\gamma_{a} \gamma^{c}\right)_{\alpha}{ }^{\delta}\left(\gamma_{b} \gamma_{c}\right)_{\beta}{ }^{\epsilon} M_{\delta \epsilon} \\
& +\frac{14}{5} \kappa\left(\gamma_{c}\right)_{\alpha \beta} K_{(a \mid b) c}+\frac{2}{5} \kappa\left(\gamma_{b}{ }^{c d}\right)_{\alpha \beta} K_{(a \mid c) d}-\frac{2}{5} \kappa\left(\gamma_{a}{ }^{c d}\right)_{\alpha \beta} K_{(b \mid c) d} .
\end{aligned}
$$

The presence of $K_{a \mid b c}$, acting as (4.47), follows from the Jacobi identity. In principle, one can derive all the required operators and their algebraic relations by computing the free Lie superalgebra of successive applications of $M_{\alpha a}$ to itself and dropping the representations not required by the Jacobi identity. Let us denote this extension of $\mathcal{H}_{L}$ by

$$
\widehat{\mathcal{H}}_{L}=\left\{M_{a b}, M_{\alpha b}, M_{\alpha \beta}, K_{a \mid b c}, K_{a, b \beta}, \cdots\right\}
$$


Unfortunately, this approach is inefficient. Already in (4.49), there are an additional five representations that could have appeared on the right-hand side. Luckily, the direct approach of building the $\Omega$ connections and determining how they must transform shortcircuited this analysis. But that is hardly an elegant solution because repeating it for higher levels would require that we introduce gauge connections, curvatures, and constraints prior to first characterizing the algebra. It would be far simpler if we could just start with an algebra that works and derive the required connections and curvatures directly.

This is what we will now propose. Recall the work of Poláček and Siegel [31], who motivated by the structure of current algebras on the string worldsheet, proposed (in the bosonic case) pairing the double Lorentz generator $M_{\hat{a} \hat{b}}$ with a dual generator $\widetilde{M}^{\hat{a} \hat{b}}$. This mirrors the pairing of $P_{\hat{a}}$ with itself (and, in the supersymmetric case, $Q_{\alpha}$ with $\left.\tilde{Q}^{\alpha}\right)$. This permits one to combine $P_{\hat{a}}, M_{\hat{a} \hat{b}}$ and $\widetilde{M}^{\hat{a} \hat{b}}$ into a single $X_{A}$ operator with an extended metric $\eta^{A B}$. The current algebra then implies that the structure constants $f_{A B}{ }^{C}$ in $\left[X_{A}, X_{B}\right]=-f_{A B}{ }^{C} X_{C}$ must be totally antisymmetric when lowered with $\eta$. As we have discussed, this amounts to the implication

$$
\left[M_{c b}, P_{a}\right]=\eta_{b a} P_{c}-\eta_{c a} P_{b} \quad \Longrightarrow \quad\left[P_{a}, P_{b}\right]=-2 \widetilde{M}_{a b}
$$

and the algebra closes on $P_{a}, M_{a b}$, and $\widetilde{M}^{a b}$. Because $P_{a}$ has dimension $1, \eta$ must pair an operator of dimension $\Delta$ with one of $2-\Delta$; we see above that $\widetilde{M}^{a b}$ is dimension 2 . This generator can be understood as the first non-trivial generator in the Maxwell $\infty$ algebra, which is the free Lie algebra beginning with the bosonic generators $P_{a}$. It extends to higher dimensions, with $\left[P_{a},\left[P_{b}, P_{c}\right]\right]$ corresponding, for example, to a reducible hook representation. (As discussed in section 2, for the bosonic case, we would further impose tracelessness.) However, one can truncate the resulting algebra to include only $P, M$ and $\widetilde{M}$, and this coincides with the algebra originally discussed in [31].

If we require the same doubling of generators in the supersymmetric case, we find that the generators of $\widehat{\mathcal{H}}_{L}$, which possess non-positive dimension $\Delta=0,-1 / 2,-1, \cdots$, have "reflections" at positive dimension $2-\Delta$ that coincide with the generators of a free Lie superalgebra that we denote super-Maxwell ${ }_{\infty}$. We list these in table 3 up through dimension 4. This superalgebra extends the supersymmetry algebra (4.44). For the details of its construction, we refer the reader to appendix C. To make complete contact, we must provide the translation table between the generators in the appendix and the duals of generators already encountered. These are

$$
\begin{aligned}
Y_{a b} & =-2 \widetilde{M}_{a b}, \quad Y_{b}^{\beta}=\widetilde{M}_{b}^{\alpha}, \quad Y^{\alpha \beta}=2 \widetilde{M}^{\alpha \beta}, \\
Y_{a \mid b c} & =-\widetilde{K}_{a \mid b c}, \quad Y_{a, b}{ }^{\beta}=-\widetilde{K}_{a, b}{ }^{\beta}, \quad Y_{, b}^{\alpha}=-\widetilde{K}_{, b}^{\alpha}, \quad Y^{\alpha \mid \beta \gamma}=-\widetilde{K}^{\alpha \mid \beta \gamma} .
\end{aligned}
$$

The generators we have denoted by $\widetilde{K}$ are dual to the generators that shift the tangent space connections $\Omega$. The connections associated to $K$ will be collectively denoted $H$. These pick up shift symmetries as well, which we denote by $L$ and their duals by $\widetilde{L}$. Following the pattern here, we will identify all higher generators with negative signs so that the appropriate $Y$ 's become $-\widetilde{K}$ or $-\widetilde{L}$. 


\begin{tabular}{c|c|c|c|c}
\hline dimension & operator & constraint & dual operator & dual dimension \\
\hline 1 & $P^{a}$ & - & $P_{a}$ & 1 \\
\hline $3 / 2$ & $\tilde{Q}^{\alpha}$ & - & $Q_{\alpha}$ & $1 / 2$ \\
\hline 2 & $\widetilde{M}^{a b}$ & antisymmetric & $M_{a b}$ & 0 \\
\hline $5 / 2$ & $\widetilde{M}^{a \beta}$ & $\gamma$-traceless & $M_{\alpha b}$ & $-1 / 2$ \\
\hline 3 & $\widetilde{M}^{\alpha \beta}$ & symmetric & $M_{\alpha \beta}$ & -1 \\
& $\widetilde{K}^{a \mid b c}$ & hook irrep & $K_{a \mid b c}$ & \\
\hline $7 / 2$ & $\widetilde{K}^{a, b \beta}$ & $\gamma$-traceless on $b \beta$ & $K_{a, b \beta}$ & $-3 / 2$ \\
\hline 4 & $\widetilde{K}^{\alpha, b \beta}$ & $\gamma$-traceless on $b \beta$ & $K_{\alpha, b \beta}$ & -2 \\
& $\widetilde{L}^{a b, c d}$ & pairwise antisymmetric & $L_{a b, c d}$ & \\
& $\widetilde{L}^{a|b| c d}$ & 21000 irrep & $L_{a|b| c d}$ & \\
\hline$\vdots$ & $\vdots$ & $\vdots$ & $\vdots$ & $\vdots$ \\
\hline
\end{tabular}

Table 3. Generators of super-Maxwell $\infty_{\infty}$ through dimension 4 and their duals. The generators at dimension zero and below form a closed algebra, which we conjecture is the extension $\widehat{\mathcal{H}}_{L}$ required for $\mathcal{N}=1$ super-DFT.

Below we give some of the algebraic relations of super-Maxwell $\infty_{\infty}$ up through dimension 4 .

(dimension 2)

$$
\left[P_{a}, P_{b}\right]=-2 \widetilde{M}_{a b}, \quad\left\{Q_{\alpha}, \tilde{Q}^{\beta}\right\}=-\frac{1}{2}\left(\gamma^{a b}\right)_{\alpha}{ }^{\beta} \widetilde{M}_{a b}
$$

(dimension 5/2)

$$
\left[P_{b}, \tilde{Q}^{\alpha}\right]=\widetilde{M}_{b}^{\alpha}, \quad\left[Q_{\alpha}, \widetilde{M}_{a b}\right]=-\kappa\left(\gamma_{[a}\right)_{\alpha \beta} \widetilde{M}_{b]}^{\beta}
$$

(dimension 3)

$$
\begin{aligned}
\left\{\tilde{Q}^{\alpha}, \tilde{Q}^{\beta}\right\} & =2 \widetilde{M}^{\alpha \beta} \\
{\left[P_{a}, \widetilde{M}_{b c}\right] } & =\frac{1}{2} \widetilde{K}_{a \mid b c}-\frac{2}{9} \kappa \eta_{a[b}\left(\gamma_{c]}\right)_{\alpha \beta} \widetilde{M}^{\alpha \beta} \\
\left\{Q_{\alpha}, \widetilde{M}_{b}^{\beta}\right\} & =-\frac{1}{4}\left(\gamma^{c d}\right)_{\alpha}{ }^{\beta} \widetilde{K}_{b \mid c d}-2 \kappa\left(\gamma_{b}\right)_{\alpha \gamma} \widetilde{M}^{\gamma \beta}+\frac{1}{9} \kappa\left(\gamma_{b c}\right)_{\alpha}{ }^{\beta}\left(\gamma^{c}\right)_{\gamma \delta} \widetilde{M}^{\gamma \delta}
\end{aligned}
$$

(dimension 7/2) $\quad\left[\tilde{Q}^{\alpha}, \widetilde{M}_{a b}\right]=-\widetilde{K}_{[a, b]}^{\alpha}, \quad\left[P_{a}, \widetilde{M}_{b}^{\beta}\right]=-\widetilde{K}_{a, b}^{\beta}$,

$$
\begin{aligned}
{\left[Q_{\alpha}, \widetilde{M}^{\beta \gamma}\right] } & =-\frac{1}{2}\left(\gamma^{a b}\right)_{\alpha}{ }^{(\beta} \widetilde{K}_{a, b}^{\gamma)}, \\
{\left[Q_{\alpha}, \widetilde{K}_{b \mid c d}\right] } & =2 \kappa\left(\gamma_{b}\right)_{\alpha \gamma} \widetilde{K}_{[c, d]}^{\gamma}+2 \kappa\left(\gamma_{[c \mid}\right)_{\alpha \gamma} \widetilde{K}_{b, \mid d]}{ }^{\gamma}
\end{aligned}
$$

(dimension 4)

$$
\begin{aligned}
\left\{\widetilde{Q}^{\alpha}, \widetilde{M}^{b \beta}\right\}= & -\widetilde{K}^{\alpha, b \beta}, \quad\left[P_{a}, \widetilde{M}^{\beta \gamma}\right]=-\widetilde{K}^{\beta}{ }_{, a}^{\gamma}, \\
{\left[P_{a}, \widetilde{K}_{b \mid c d}\right]=} & \widetilde{L}_{a|b| c d}+\frac{3}{4} \widetilde{L}_{a b, c d}+\frac{3}{8} \eta_{a c} \eta^{e f} \widetilde{L}_{b e, d f} \\
& -\frac{3}{10} \kappa \eta_{a c}\left(\gamma_{b}\right)_{\alpha \beta} \widetilde{K}_{, d}^{\alpha}+\left.\frac{7}{10} \kappa \eta_{a c}\left(\gamma_{d}\right)_{\alpha \beta} \widetilde{K}_{, b}^{\alpha}\right|_{p r o j}, \\
\left\{Q_{\alpha}, \widetilde{K}_{a, b}{ }^{\beta}\right\}= & -\kappa\left(\gamma_{a}\right)_{\alpha \gamma} \widetilde{K}_{,{ }^{\beta}}^{\beta}-\kappa\left(\gamma_{b}\right)_{\alpha \gamma} \widetilde{K}_{, a}^{\gamma}-\kappa\left(\gamma_{b}\right)_{\alpha \gamma} \widetilde{K}_{, a}^{\beta}{ }_{, a}^{\gamma} \\
& +\frac{1}{9} \kappa\left(\gamma_{b c}\right)_{\alpha}{ }^{\beta}\left(\gamma^{c}\right)_{\gamma \delta} \widetilde{K}_{, a}^{\gamma}+\frac{1}{4}\left(\gamma^{c d}\right)_{\alpha}{ }^{\beta}\left[P_{a}, \widetilde{K}_{b \mid c d}\right]
\end{aligned}
$$

The above relations can be "reflected" to derive the action of the untilded generators. We 
give some of these results below, grouped again by dimension.

(dimension 0)

$$
\begin{aligned}
\left\{M_{\gamma c}, Q_{\alpha}\right\} & =-\left.\kappa\left(\gamma^{b}\right)_{\gamma \alpha} M_{b c}\right|_{\text {proj }}, \\
{\left[M_{\alpha \beta}, P_{a}\right] } & =-\frac{2}{9} \kappa\left(\gamma^{b}\right)_{\alpha \beta} M_{a b}, \quad\left[K_{a \mid b c}, P_{d}\right]=\left.\frac{1}{2} \eta_{a d} M_{b c}\right|_{\text {proj }}, \\
\left\{K_{a, b \beta}, Q^{\gamma}\right\} & =-\left.\delta_{\beta}^{\gamma} M_{a b}\right|_{\text {proj }}
\end{aligned}
$$

(dimension $-1 / 2)$

$$
\begin{aligned}
{\left[M_{\beta \gamma}, Q_{\alpha}\right] } & =-2 \kappa\left(\gamma^{b}\right)_{\alpha \beta} M_{\gamma b}+\left.\frac{1}{9} \kappa\left(\gamma^{b}\right)_{\beta \gamma} M_{\alpha b}\right|_{\text {proj }} \\
{\left[K_{b \mid c d}, Q_{\alpha}\right] } & =-\left.\frac{1}{4}\left(\gamma_{c d}\right)_{\alpha}{ }^{\beta} M_{\beta b}\right|_{\text {proj }} \\
{\left[K_{c, b \beta}, P_{a}\right] } & =-\eta_{c a} M_{\beta b}, \quad\left[K_{\gamma, b \beta}, Q^{\alpha}\right]=-\delta_{\gamma}{ }^{\alpha} M_{b \beta}
\end{aligned}
$$

(dimension -1)

$$
\begin{aligned}
\left\{K_{c, b \beta}, Q_{\alpha}\right\} & =-\frac{1}{2}\left(\gamma_{c b}\right)_{\alpha}^{\gamma} M_{\gamma \beta}+2 \kappa\left(\gamma^{d}\right)_{\alpha \beta} K_{d \mid c b}-\left.2 \kappa\left(\gamma^{d}\right)_{\alpha \beta} K_{c \mid b d}\right|_{\text {proj }} \\
{\left[L_{e|b| c d}, P_{a}\right] } & =\left.\eta_{a e} K_{b \mid c d}\right|_{\text {proj }}, \\
{\left[L_{d e, b c}, P_{a}\right] } & =\frac{3}{4} \eta_{a d} K_{e \mid b c}+\left.\frac{3}{8} \eta_{e c} K_{d \mid a b}\right|_{\text {proj }}, \\
{\left[K_{\gamma, b \beta}, P_{a}\right] } & =-\eta_{b a} M_{\gamma \beta}+\frac{3}{10} \kappa\left(\gamma^{d}\right)_{\gamma \beta} K_{d \mid b a}+\left.\frac{3}{10} \kappa\left(\gamma^{d}\right)_{\gamma \beta} K_{b \mid a d}\right|_{\text {proj }}
\end{aligned}
$$

Other commutators can be determined from the Jacobi identity.

This Lie superalgebra, which is an extension of the super-Maxwell $l_{\infty}$ algebra, would seem to continue to infinity in both directions. A separate construction of it is sketched in appendix C.2. Unlike the super-Maxwell $\infty$ algebra itself, which possesses only positive generators, the extension above includes negative dimensions. This would seem to make it difficult to consistently truncate the algebra, although we know of no proof of this. The algebraic relations implied down through dimension -1 do coincide with what a direct construction of connections, curvatures, etc. would give. But we leave it an open question as to whether the algebra required is actually the full extension of super-Maxwell $\infty$ discussed above.

\subsection{New connections, transformations, and curvatures}

Now that we have a proposal for the algebra we want to gauge (at least up through dimension 4 and down to dimension -2), we may go about introducing connections and curvatures. The idea is to match the existing results for the torsion tensor and $\Omega$ connection from section 4.2 and to extend them by introducing new $H$ connections, the superspace analogues of the $h$ connection discussed in section 2 .

First, we adopt the condensed notation of appendix B, here extended to the superspace case. We denote the supertranslation generators by $P_{\mathcal{A}}$, the $\widehat{\mathcal{H}}_{L} \times \widehat{\mathrm{SO}(9,1)}{ }_{R}$ generators by $X_{\underline{a}}$, and their duals by $\widetilde{X}^{\underline{a}}$. Following appendix B, we use $\underline{a}, \underline{b}, \cdots$ here to denote $\left.\widehat{\mathcal{H}}=\widehat{\mathcal{H}}_{L} \times \mathrm{S} \widehat{\mathrm{O}(9,1)}\right)_{R}$ indices. We hope this will cause no confusion with the tangent space 
indices $(a, \bar{a})$. Relative to the previous subsection, we're now including the right-handed $P$ and the $\widehat{\mathrm{SO}(9,1})_{R}$ generators, and their duals, which commute with $P_{A}, \widehat{\mathcal{H}}_{L}$ and its dual. The rigid algebra can be written

$$
\begin{aligned}
& {\left[P_{\mathcal{A}}, P_{\mathcal{B}}\right]=-f_{\mathcal{A B}}{ }^{\mathcal{C}} P_{\mathcal{C}}-\tilde{X}^{\underline{c}} f_{\underline{c} \mathcal{A} \mathcal{B}},} \\
& {\left[X_{\underline{a}}, P_{\mathcal{B}}\right]=-f_{\underline{a} \mathcal{B}}{ }^{\mathcal{C}} P_{\mathcal{C}}-f_{\underline{a}} \mathcal{B}^{\underline{c}} X_{\underline{c}},} \\
& {\left[X_{\underline{a}}, X_{\underline{b}}\right]=-f_{\underline{a b}} \underline{c} X_{\underline{c}},} \\
& {\left[\tilde{X}^{\underline{a}}, \tilde{X}^{\underline{b}}\right]=-\widetilde{X}^{\underline{c}} f_{\underline{c}}^{\underline{a b}},} \\
& {\left[P_{\mathcal{A}}, \widetilde{X}^{\underline{b}}\right]=-\widetilde{X}^{\underline{c}} f_{\underline{c} \mathcal{A}^{\underline{b}}},} \\
& {\left[X_{\underline{a}}, \widetilde{X}^{\underline{b}}\right]=-\widetilde{X}^{\underline{c}} f_{\underline{c} \underline{a}^{\underline{b}}}-f_{\underline{a}}{ }^{\underline{b}} P_{\mathcal{C}}-f_{\underline{a}} \underline{b c} X_{\underline{c}} .}
\end{aligned}
$$

A more compact form is

$$
\left[X_{\widehat{\mathcal{A}}}, X_{\widehat{\mathcal{B}}}\right]=-f_{\widehat{\mathcal{A}} \widehat{\mathcal{B}}} \widehat{\widehat{\mathcal{C}}} X_{\widehat{\mathcal{C}}}
$$

for $X_{\widehat{\mathcal{A}}}=\left(X_{\underline{a}}, P_{\mathcal{A}}, \tilde{X}^{\underline{a}}\right)$ and where $f_{\widehat{\mathcal{A} \widehat{\mathcal{B}} \widehat{\mathcal{C}}}}=f_{\widehat{\mathcal{A}} \widehat{\mathcal{B}}} \widehat{\widehat{\mathcal{D}}} \eta_{\widehat{\mathcal{D}} \widehat{\mathcal{C}}}$ is totally antisymmetric with $\eta_{\widehat{\mathcal{A}} \widehat{\mathcal{B}}}$ given by

$$
\eta_{\widehat{\mathcal{A} \widehat{\mathcal{B}}}}=\left(\begin{array}{ccc}
0 & 0 & \delta_{\underline{a}^{\underline{b}}} \\
0 & \eta_{\mathcal{A B \mathcal { B }}} & 0 \\
(-1)^{\underline{a} b} \delta_{\underline{\underline{b}}} \underline{\underline{a}} & 0 & 0
\end{array}\right) .
$$

Following the discussion in appendix B, we introduce the dilaton $\Phi$, the vielbein $\mathcal{V}_{\mathcal{M}}{ }^{\mathcal{A}}$, connections $H_{\mathcal{M}^{\underline{a}}}$, and an additional graded antisymmetric field $P^{\underline{a b}}$. These transform under diffeomorphisms and gauge transformations (with parameter $\Lambda^{\underline{a}}$ ) as ${ }^{10}$

$$
\begin{aligned}
& \delta \Phi=\mathbb{L}_{\xi} \Phi, \\
& \delta \mathcal{V}_{\mathcal{M}}^{\mathcal{A}}=\mathbb{L}_{\xi} \mathcal{V}_{\mathcal{M}}^{\mathcal{A}}+\mathcal{V}_{\mathcal{M}}^{\mathcal{B}} \Lambda^{\underline{c}} f_{\underline{c}} \mathcal{B}^{\mathcal{A}}, \\
& \delta H_{\mathcal{M}} \underline{\underline{a}}=\mathbb{L}_{\xi} H_{\mathcal{M}^{\underline{a}}}+\partial_{\mathcal{M}} \Lambda^{\underline{a}}+H_{\mathcal{M}}^{\underline{\underline{b}}} \Lambda^{\underline{\underline{c}}} f_{\underline{c} \underline{\underline{a}}}^{\underline{a}}+\mathcal{V}_{\mathcal{M}}^{\mathcal{B}} \Lambda^{\underline{c}} f_{\underline{c} \mathcal{B}^{\underline{a}}}^{\underline{a}}, \\
& \delta P^{\underline{a b}}=\xi^{\mathcal{M}} \partial_{\mathcal{M}} P^{\underline{a b}}-\Lambda^{\underline{c}} f_{\underline{c}}^{\underline{a b}}-2 \Lambda^{\underline{c}} P^{\underline{d}}\left[\underline{a} f_{\underline{c d}} \underline{b}^{\underline{b}}-H^{\mathcal{M}[\underline{a}} \partial_{\mathcal{M}} \Lambda^{\underline{b}]}-\Lambda^{\underline{c}} H^{\mathcal{D}[\underline{a}} f_{\mathcal{D}_{\underline{c}}}{ }^{\underline{b}} .\right.
\end{aligned}
$$

The connection $H_{\mathcal{M}}$ a generalizes $\Omega_{\mathcal{M}}{ }^{\mathcal{A B}}$ and the field $P^{a b}$ generalizes the one introduced by Polàček and Siegel [31]. Using these ingredients, we can construct covariant derivatives

$$
\nabla_{\mathcal{A}}=\mathcal{V}_{\mathcal{A}}^{\mathcal{M}} \partial_{\mathcal{M}}-H_{\mathcal{A}^{\underline{b}}} X_{\underline{b}}, \quad \widetilde{\nabla}^{\underline{a}}=H^{\mathcal{M} \underline{a}} \partial_{\mathcal{M}}+\left(P^{\underline{a} \underline{b}}-\frac{1}{2} H^{\mathcal{M} \underline{a}} H_{\mathcal{M}^{\underline{b}}}\right) X_{\underline{b}} .
$$

These correspond to the curved extensions of $P_{\mathcal{A}}$ and $\widetilde{X}^{\underline{a}}$. Their algebra can again be written (4.56), but with some of the components of $f$ now becoming structure functions. These are the four curvatures $\mathcal{T}_{\mathcal{A B C}}, \mathcal{R}_{\mathcal{A B}}{ }^{c}, \mathcal{R}_{\mathcal{A}}{ }^{b c}$, and $\mathcal{R}^{a b c}$, which appear in the curved

\footnotetext{
${ }^{10}$ For purposes of legibility, we have suppressed gradings in these and subsequent expressions.
} 
algebra as

$$
\begin{aligned}
& {\left[\nabla_{\mathcal{A}}, \nabla_{\mathcal{B}}\right]=-\mathcal{T}_{\mathcal{A B}}{ }^{\mathcal{C}} \nabla_{\mathcal{C}}-\mathcal{R}_{\mathcal{A B}}{ }^{\underline{c}} X_{\underline{c}}-\widetilde{\nabla}^{\underline{c}} f_{\underline{c} \mathcal{A B}},} \\
& {\left[X_{\underline{a}}, \nabla_{\mathcal{B}}\right]=-f_{\underline{a} \mathcal{B}^{\mathcal{C}}} \nabla_{\mathcal{C}}-f_{\underline{a} \mathcal{B}^{\underline{c}}} X_{\underline{c}},} \\
& {\left[X_{\underline{a}}, X_{\underline{b}}\right]=-f_{\underline{a b}} \underline{\underline{c}} X_{\underline{c}},} \\
& {\left[\widetilde{\nabla}^{\underline{a}}, \widetilde{\nabla}^{\underline{b}}\right]=-\widetilde{\nabla}^{\underline{c}} f_{\underline{c}}^{\underline{a b}}-\mathcal{R}^{\underline{a b} \underline{C}} \nabla_{\mathcal{C}}-\mathcal{R}^{\underline{a b c}} X_{\underline{c}},} \\
& {\left[\nabla_{\mathcal{A}}, \widetilde{\nabla}^{\underline{b}}\right]=-\widetilde{\nabla}^{\underline{c}} f_{\underline{c} \mathcal{A}} \underline{\underline{b}}-\mathcal{R}_{\mathcal{A}} \underline{\underline{b} \mathcal{C}} \nabla_{\mathcal{C}}-\mathcal{R}_{\mathcal{A}} \underline{\underline{b c}} X_{\underline{c}},} \\
& {\left[X_{\underline{a}}, \widetilde{\nabla} \underline{b}\right]=-\widetilde{\nabla}^{\underline{c}} f_{\underline{c a}}^{\underline{b}}-f_{\underline{\underline{a}}}^{\underline{b}} \nabla_{\mathcal{C}}-f_{\underline{a}} \underline{\underline{b} c} X_{\underline{c}} .}
\end{aligned}
$$

The torsion tensor is given as

$$
\mathcal{T}_{\mathcal{C B} \mathcal{A}}=-3 \nabla_{[\mathcal{C}} \mathcal{V}_{\mathcal{B}}{ }^{\mathcal{M}} \mathcal{V}_{\mathcal{M A}]}, \quad \nabla_{\mathcal{C}} \mathcal{V}_{\mathcal{B}}{ }^{\mathcal{M}}:=D_{\mathcal{C}} \mathcal{V}_{\mathcal{B}}{ }^{\mathcal{M}}+H_{\mathcal{C}} f_{\underline{d}} \mathcal{B}^{\mathcal{A}} \mathcal{V}_{\mathcal{A}}{ }^{\mathcal{M}}
$$

The curvature tensor $\mathcal{R}_{\mathcal{C B}}{ }^{a}$ is

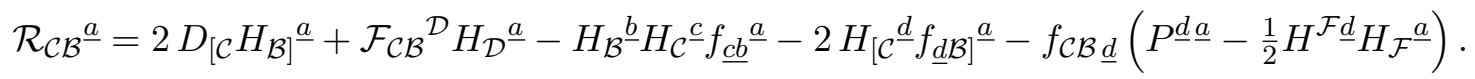

The other curvature tensors $\mathcal{R}_{\mathcal{A}} \underline{\underline{b c}}$ and $\mathcal{R}^{a b c}$ correspond to covariantizations of $\nabla_{\mathcal{A}} P^{\underline{b c}}$ and $\widetilde{\nabla}[\underline{a} P \underline{b c}]$ and are discussed further in appendix B. In addition to these, one has dilatondependent curvatures

$$
\begin{aligned}
& \mathcal{T}_{\mathcal{A}}=\nabla_{\mathcal{A}} \log \Phi+\nabla_{\mathcal{M}} \mathcal{V}_{\mathcal{A}}{ }^{\mathcal{M}}+f_{\mathcal{A}} \underline{\underline{b}}, \\
& \mathcal{R}^{\underline{a}}=D^{\mathcal{B}} H_{\mathcal{B}^{\underline{a}}}+\mathcal{F}^{\mathcal{B}} H_{\mathcal{B}^{\underline{a}}}-H^{\mathcal{B} \underline{c}} f_{\underline{c} \mathcal{B}^{\underline{a}}}+P^{\underline{b c}} f_{\underline{c} \underline{\underline{a}}}^{\underline{a}}+f_{\underline{b}} \underline{\underline{b} a}
\end{aligned}
$$

where $\mathcal{F}_{\mathcal{A}}:=D_{\mathcal{A}} \log \Phi+\partial_{\mathcal{M}} \mathcal{V}_{\mathcal{A}}{ }^{\mathcal{M}}$

Using these expressions, we can now give explicit expressions for the curvatures. The transformation rules for $\Omega$ and the expressions for the torsion tensors reproduce what we found by direct construction in section 4.2 , so we do not repeat that discussion here. We turn our attention directly to the $\Omega$ curvatures. We restrict attention to components of curvatures only through dimension 2 , which is sufficient for understanding the two-derivative DFT action.

First, we take the curvature $\mathcal{R}_{\mathcal{A B} \overline{B d}}$, which will have the simplest structure:

$$
\begin{aligned}
& \mathcal{R}_{\alpha \beta \overline{c d}}=R(\Omega)_{\alpha \beta \overline{c d}} \\
& \mathcal{R}_{\alpha \bar{b} \overline{c d}}=R(\Omega)_{\alpha \bar{b} \overline{c d}}-H_{\alpha \bar{b} \mid \overline{c d}} \\
& \mathcal{R}_{\alpha b \overline{c d}}=R(\Omega)_{\alpha b \overline{c d}} \\
& \mathcal{R}_{a \bar{b} \overline{c d}}=R(\Omega)_{a \overline{b c d}}-H_{a \bar{b} \mid \overline{c d}} \\
& \mathcal{R}_{\overline{a b} \overline{c d}}=R(\Omega)_{\overline{a b} \overline{c d}}-H_{\bar{a}, \bar{b} \mid \overline{c d}}+H_{\bar{b}, \bar{a} \mid \overline{c d}}-P_{\overline{a b} \overline{c d}} \\
& \mathcal{R}_{a b \overline{c d}}=R(\Omega)_{a b \overline{c d}}-P_{a b \overline{c d}} \\
& \mathcal{R}_{\alpha}{ }^{\beta} \overline{c d}=R(\Omega)_{\alpha}{ }^{\beta} \overline{c d}-\frac{1}{4}\left(\gamma^{a b}\right)_{\alpha}{ }^{\beta} P_{a b \overline{c d}} .
\end{aligned}
$$


There remaining pieces $\mathcal{R}_{a}{ }^{\beta} \overline{c d}, \mathcal{R}_{\bar{a}}{ }^{\beta} \overline{c d}$, and $\mathcal{R}^{\alpha \beta} \overline{c d}$ are higher dimension, so we ignore them. In the above expressions, $R(\Omega)_{\mathcal{A B C D}}$ is the naive curvature built from $\Omega$, which is the superspace analogue of (2.24),

$$
R(\Omega)_{\mathcal{A B C D}}:=2 D_{[\mathcal{A}} \Omega_{\mathcal{B}] \mathcal{C D}}-2 \Omega_{[\mathcal{A} \mid \mathcal{C}} \Omega_{\mid \mathcal{B}] \mathcal{E D}}+\mathcal{F}_{\mathcal{A B}}{ }^{\mathcal{E}} \Omega_{\mathcal{E C D}}+\frac{1}{2} \Omega^{\mathcal{E}}{ }_{\mathcal{A B}} \Omega_{\mathcal{E C D}}
$$

The connections $H_{\mathcal{A} \bar{b} \mid \overline{c d}}$ are necessary to gauge the $\Lambda_{\bar{b} \mid \overline{c d}}$ shift symmetry and the fields $P$ are required to cure the Lorentz non-covariance of $R(\Omega)_{\mathcal{A B C D}}$ when both $\mathcal{A B}$ and $\mathcal{C D}$ correspond to tangent space transformations. All of this is a straightforward extension of the bosonic case (except for $\mathcal{R}_{\alpha}{ }^{\beta} \overline{c d}$ ).

The curvature $\mathcal{R}_{\mathcal{A B} c d}$ is already a good deal more complicated:

$$
\begin{aligned}
\mathcal{R}_{\alpha \beta c d} & =R(\Omega)_{\alpha \beta c d}+4 \kappa\left(\gamma_{[c}\right)_{\gamma(\beta} \Omega_{\alpha) d]}{ }^{\gamma}, \\
\mathcal{R}_{\alpha \bar{b} c d} & =R(\Omega)_{\alpha \bar{b} c d}-2 \kappa\left(\gamma_{[c}\right)_{\alpha \gamma} \Omega_{\bar{b} d]}{ }^{\prime} \\
\mathcal{R}_{\alpha b c d} & =R(\Omega)_{\alpha b c d}+\frac{2}{9} \kappa \eta_{b[c}\left(\gamma_{d]}\right)_{\gamma \delta} \Omega_{\alpha}{ }^{\gamma \delta}-2 \kappa\left(\gamma_{[c \mid}\right)_{\alpha \gamma} \Omega_{b \mid d]}{ }^{\gamma}-H_{\alpha b \mid c d} \\
\mathcal{R}_{\overline{a b} c d} & =R(\Omega)_{\overline{a b} c d}-P_{\overline{a b} c d} \\
\mathcal{R}_{a \bar{b} c d} & =R(\Omega)_{a \bar{b} c d}-\frac{2}{9} \kappa \eta_{a[c}\left(\gamma_{d]}\right)_{\alpha \beta} \Omega_{\bar{b}}{ }^{\alpha \beta}+H_{\bar{b} a \mid c d} \\
\mathcal{R}_{a b c d} & =R(\Omega)_{a b c d}+\frac{4}{9} \kappa \Omega_{[a}{ }^{\alpha \beta} \eta_{b][c}\left(\gamma_{d]}\right)_{\alpha \beta}-H_{a, b \mid c d}+H_{b, a \mid c d}-P_{a b c d} \\
\mathcal{R}_{\alpha}{ }^{\beta} c d & =R(\Omega)_{\alpha}{ }^{\beta} c d+H_{\alpha c, d^{\beta}}-H_{\alpha d, c}{ }^{\beta}-\frac{1}{4}\left(\gamma^{a b}\right)_{\alpha}{ }^{\beta} P_{a b c d} .
\end{aligned}
$$

The new $\kappa$-dependent modifications involving $\Omega_{\mathcal{A} b}{ }^{\gamma}$ and $\Omega_{\mathcal{A}}^{\beta \gamma}$ are required to ensure covariance under $\lambda_{b}{ }^{\gamma}$ and $\lambda^{\beta \gamma}$ transformations.

The curvature $\mathcal{R}_{\mathcal{A B} c^{\gamma}}$ does not have a bosonic counterpart. Its structure is fairly intricate, but only the lowest few pieces have a sufficiently small dimension to be relevant for us:

$$
\begin{aligned}
& \mathcal{R}_{\alpha \beta c}{ }^{\gamma}=R(\Omega)_{\alpha \beta c^{\gamma}}-2 \kappa\left(\gamma_{c}\right)_{\delta(\beta} \Omega_{\alpha)} \delta \gamma+\frac{1}{9} \kappa\left(\gamma_{c d}\right)_{(\beta \mid}^{\gamma}\left(\gamma^{d}\right)_{\delta \epsilon} \Omega_{\mid \alpha)} \delta \epsilon-\frac{1}{2}\left(\gamma^{d e}\right)_{(\beta}{ }^{\gamma} H_{\alpha) c \mid d e} \\
& \mathcal{R}_{\alpha \bar{b} c}{ }^{\gamma}=R(\Omega)_{\alpha \bar{b} c}{ }^{\gamma}+\kappa\left(\gamma_{c}\right)_{\alpha \delta} \Omega_{\bar{b}}{ }^{\delta \gamma}+\frac{1}{4}\left(\gamma^{e f}\right)_{\alpha}^{\gamma} H_{\bar{b} c \mid e f}, \\
& \mathcal{R}_{\alpha b c}{ }^{\gamma}=R(\Omega)_{\alpha b c}{ }^{\gamma}+\kappa\left(\gamma_{c}\right)_{\alpha \delta} \Omega_{b}^{\delta \gamma}-\frac{1}{18} \kappa\left(\gamma_{c d}\right)_{\alpha}^{\gamma}\left(\gamma^{d}\right)_{\delta \epsilon} \Omega_{b} \delta \epsilon+\frac{1}{4}\left(\gamma^{e f}\right)_{\alpha}^{\gamma} H_{b, c \mid e f}-H_{\alpha b, c}{ }^{\gamma} .
\end{aligned}
$$

Again, $\Omega_{\mathcal{A} b}{ }^{\gamma}$ and $\Omega_{\mathcal{A}}^{\beta \gamma}$ contributions are required for $\lambda$-covariance, and similarly for the $H$ connections. In the last expression we find a new connection $H_{\mathcal{A} b, c}{ }^{\gamma}$, which gauges the $\Lambda_{b, c}{ }^{\gamma}$ shift symmetry of $\Omega_{b, c}{ }^{\gamma}$. While there are $P_{\mathcal{A B}}{ }^{\gamma}$ contributions at higher dimension when $\mathcal{A B}$ corresponds to a generator, none appear here.

For $\mathcal{R}_{\mathcal{A B}}{ }^{\gamma \delta}$, only the lowest component is relevant:

$$
\mathcal{R}_{\alpha \beta}{ }^{\gamma \delta}=R(\Omega)_{\alpha \beta}{ }^{\gamma \delta}-2 H_{(\alpha b, c}{ }^{(\gamma}\left(\gamma^{b c}\right)_{\beta)}{ }^{\delta)}
$$


The remaining curvatures are the dilaton-dependent ones $\mathcal{R}^{\underline{a}}$. At dimension 2 , these consist of

$$
\begin{aligned}
& \mathcal{R}_{a b}=R(\Omega)_{a b}+2 \kappa\left(\gamma_{a}\right)_{\alpha \beta} \Omega^{\alpha}{ }_{b}{ }^{\beta}-\frac{2}{9} \kappa\left(\gamma_{[a}\right)_{\alpha \beta} \Omega_{b]}{ }^{\alpha \beta}-H_{c \mid a b}^{c}+2 H_{\gamma[a, b]}{ }^{\gamma}, \\
& \mathcal{R}_{\overline{a b}}=R(\Omega)_{\overline{a b}}+H_{\bar{c} \mid \overline{a b}}^{\bar{c}}
\end{aligned}
$$

where $R(\Omega)_{\mathcal{A B}}=D^{\mathcal{C}} \Omega_{\mathcal{C} \mathcal{A B}}+\mathcal{F}^{\mathcal{C}} \Omega_{\mathcal{C} \mathcal{A B}}$.

We do not give the $H$ transformations explicitly, but they can be derived from (4.58c). As with the $\Omega$ connections, we can use the $H$ connections and $P$ fields to impose a number of constraints on $\mathcal{R}$ :

$$
\begin{aligned}
& \left.H_{\alpha \bar{b} \mid \overline{c d}} \quad \Longrightarrow \quad \mathcal{R}_{\alpha \bar{b} \overline{c d}}\right|_{\bar{b} \mid \overline{c d}}=0 \\
& \left.H_{\alpha b \mid c d} \quad \Longrightarrow \quad \mathcal{R}_{\alpha b c d}\right|_{b \mid c d}=0 \\
& \left.H_{\bar{a} b \mid c d} \quad \Longrightarrow \quad \mathcal{R}_{\bar{a} b c d}\right|_{b \mid c d}=0 \\
& \left.H_{a \bar{b} \mid \overline{c d}} \quad \Longrightarrow \quad \mathcal{R}_{a \bar{b} \bar{c} \bar{b}}\right|_{\bar{b} \mid \overline{c d}}=0 \\
& P_{a b \overline{c d}} \quad \Longrightarrow \quad \mathcal{R}_{a b \overline{c d}}=\mathcal{R}_{\overline{c d} a b}, \\
& H_{a, b \mid c d}, P_{a b c d} \quad \Longrightarrow \quad \mathcal{R}_{a b c d}=\frac{1}{45} \eta_{a[c} \eta_{d] b} \mathcal{R}+\mathcal{R}_{[a b c d]}, \\
& H_{\bar{a}, \bar{b} \mid \overline{c d}}, P_{\overline{a b} \overline{c d}} \quad \Longrightarrow \quad \mathcal{R}_{\overline{a b} \overline{c d}}=\frac{1}{45} \eta_{\bar{a}[\bar{c}} \eta_{\bar{d}] \bar{b}} \overline{\mathcal{R}}+\mathcal{R}_{[\overline{a b} \overline{c d}]}, \\
& H_{\alpha b, c}^{\gamma} \quad \Longrightarrow \quad \mathcal{R}_{\alpha b c}^{\gamma}=0
\end{aligned}
$$

Above, we have used $\mathcal{R}=\mathcal{R}_{a b}{ }^{a b}$ and $\overline{\mathcal{R}}=\mathcal{R}_{\overline{a b}}{ }^{\overline{a b}}$.

While these constraints fully determine $P$, there remain three irreps of $H_{a, b \mid c d}$ that are undetermined. As in the bosonic case, these are the $\square \square$ component corresponding to $L_{a|b| c d}$ and the $\square+\square$ components corresponding to $L_{a b, c d}$.

\subsection{Solution of the Bianchi identities through dimension 2}

Our last task in superspace is to analyze the torsion Bianchi identities to determine what the unfixed pieces of the $\mathcal{R}(\Omega)$ curvatures are. Here we will again restrict our attention through dimension 2 .

The Bianchi identities for the torsion tensor read

$$
\begin{aligned}
& 0=\mathcal{B}_{\mathcal{A B C}} \equiv\left[4 \nabla_{\mathcal{A}} \mathcal{T}_{\mathcal{B C D}}+3 \mathcal{T}_{\mathcal{A B}}{ }^{\mathcal{E}} \mathcal{T}_{\mathcal{E} \mathcal{C D}}-6 \mathcal{R}_{\mathcal{A B C D}}\right]_{[\mathcal{A B C D}]} \\
& 0=\mathcal{B}_{\mathcal{A B}} \equiv\left[2 \nabla_{\mathcal{A}} \mathcal{T}_{\mathcal{B}}+\mathcal{T}_{\mathcal{A B}}{ }^{\mathcal{C}} \mathcal{T}_{\mathcal{C}}+\nabla^{\mathcal{C}} \mathcal{T}_{\mathcal{C} \mathcal{B}}-\mathcal{R}_{\mathcal{A}}{ }^{\mathcal{D}}{ }_{\mathcal{D B}}+\mathcal{R}_{\mathcal{B}}{ }^{\mathcal{D}}{ }_{\mathcal{D} \mathcal{A}}-\mathcal{R}_{\mathcal{A B}}\right]_{[\mathcal{A B}]} \\
& 0=\mathcal{B} \equiv \nabla^{\mathcal{A}} \mathcal{T}_{\mathcal{A}}+\frac{1}{2} \mathcal{T}^{\mathcal{A}} \mathcal{T}_{\mathcal{A}}+\frac{1}{12} \mathcal{T}^{\mathcal{A B C}} \mathcal{T}_{\mathcal{C B} \mathcal{A}}-\frac{1}{2} \mathcal{R}^{\mathcal{A B}}{ }_{\mathcal{B} \mathcal{A}}
\end{aligned}
$$

The torsion tensors are given in table 2, where we have listed the conventional and physical constraints.

The dimension $\frac{1}{2}$ torsions that we have not yet fixed are $\mathcal{T}_{\alpha \beta}{ }^{\gamma}$ and $\mathcal{T}_{\alpha}$. From the $\alpha \beta \gamma d$ component of (4.71a), we can show that $\mathcal{T}_{\alpha \beta}{ }^{\gamma}$ must obey $\left.\left(\gamma_{d}\right)_{\delta(\gamma} \mathcal{T}_{\alpha \beta}\right)^{\delta}=0$, which implies 
$\left.\mathcal{T}_{\alpha \beta} \gamma=X_{(\alpha} \delta_{\beta}\right)^{\gamma}-\frac{1}{2}\left(\gamma^{c}\right)_{\alpha \beta}\left(\gamma_{c}\right)^{\gamma \delta} X_{\delta}$ in terms of some covariant field $X_{\alpha}$. If we had not already accounted for the dilatino in the supervielbein, this is where it would appear. To avoid introducing more degrees of freedom, we fix this dimension- $1 / 2$ piece to vanish. The $\alpha \beta \gamma \bar{d}$ component of (4.71a) trivially vanishes. If we hadn't already set $\mathcal{T}_{\alpha b \bar{c}}$ to zero, we would have found that $\left(\gamma^{c}\right)_{(\alpha \beta} \mathcal{T}_{\gamma) c \bar{d}}=0$ implies that $\mathcal{T}_{\alpha b \bar{c}} \propto\left(\gamma_{b}\right)_{\alpha \beta} \mathcal{W}^{\beta}{ }_{\bar{c}}$ in terms of a gaugino superfield. We reiterate that for heterotic DFT, if the gaugino were not contained within the supervielbein, this is where it would appear. That leaves $\mathcal{T}_{\alpha}$. Although it's plausible that we could deduce a constraint on this by looking at dimension 1 Bianchi identities, we are instead simply going to impose that it vanishes. This gives a full set of vanishing dimension- $\frac{1}{2}$ physical constraints,

$$
\mathcal{T}_{\alpha \beta}^{\gamma}=\mathcal{T}_{\alpha}=\mathcal{T}_{\alpha b \bar{c}}=0
$$

At dimension 1, we first analyze various components of (4.71a). These rapidly yield constraints:

$$
\begin{aligned}
& \mathcal{B}_{\alpha \beta \overline{c d}}=0 \quad \Longrightarrow \quad \mathcal{R}_{\alpha \beta \overline{c d}}=0 \\
& \mathcal{B}_{\alpha \beta c \bar{d}}=0 \quad \Longrightarrow \quad \mathcal{T}_{\bar{a} \beta} \gamma=0 \text {, } \\
& \mathcal{B}_{\alpha \beta c d}=0 \quad \Longrightarrow \quad \mathcal{R}_{\alpha \beta c d}=-\frac{2}{5} \kappa \mathcal{T}_{\gamma(\alpha}\left(\gamma_{c d}\right)_{\beta)}{ }^{\gamma} \text {. }
\end{aligned}
$$

Next using $\mathcal{B}_{\alpha \beta \gamma}{ }^{\delta}=0$, one can show that $\mathcal{T}_{\alpha \beta}$ must be purely a vector. However, $\mathcal{B}_{\alpha \beta}=0$ is nonzero if $\mathcal{T}_{\alpha \beta}$ is purely vectorial. Thus we find that

$$
\mathcal{B}_{\alpha \beta \gamma}{ }^{\delta}=\mathcal{B}_{\alpha \beta}=0 \quad \Longrightarrow \quad \mathcal{R}_{\alpha \beta c d}=0, \quad \mathcal{T}_{a \beta}{ }^{\gamma}=\frac{1}{10}\left(\gamma_{a}\right)^{\gamma \alpha} \mathcal{T}_{\alpha \beta}=0
$$

This is as expected: there are no covariant dimension 1 fields or curvatures in supersymmetric DFT.

At dimension $\frac{3}{2}$, there are more terms to analyze but fewer spinor indices, so the group theory is simpler. We rapidly find

$$
\begin{aligned}
& \mathcal{B}_{\alpha \overline{b c d}}=0 \quad \Longrightarrow \quad \mathcal{R}_{\alpha[\bar{b} \overline{c d}]}=0 \text {, } \\
& \mathcal{B}_{\alpha b \overline{c d}}=0 \quad \Longrightarrow \quad \mathcal{R}_{\alpha b \overline{c d}}=0 \text {, } \\
& \mathcal{B}_{\alpha \bar{b} c d}=0 \quad \Longrightarrow \quad \mathcal{R}_{\alpha \bar{b} c d}=\frac{1}{5} \kappa\left(\gamma_{c d}\right)_{\alpha}{ }^{\beta} \mathcal{T}_{\beta \bar{b}}, \\
& \mathcal{B}_{\alpha b c d}=0 \quad \Longrightarrow \quad \mathcal{R}_{\alpha[b c d]}=0 \text {. }
\end{aligned}
$$

The component $\mathcal{T}_{\beta \bar{b}}$ of $\mathcal{T}_{a \bar{b}}{ }^{\gamma}$ is the covariantized gravitino field equation. Using $\mathcal{B}_{\alpha \beta \bar{c}}{ }^{\delta}=0$, one can show that it vanishes, fixing $\mathcal{R}_{\alpha \bar{b} c d}=0$ and $\mathcal{T}_{a \bar{b}} \gamma=0$. This places the component theory on-shell. Next we find that

$$
\mathcal{B}_{\alpha \beta c}{ }^{\delta}=0 \quad \Longrightarrow \quad \mathcal{R}_{\alpha \beta c}{ }^{\gamma}=\frac{1}{2}\left(\gamma^{a b}\right)_{(\beta}{ }^{\gamma} \mathcal{R}_{\alpha) c a b}
$$

This is a powerful constraint because $\mathcal{R}_{\alpha \beta c}{ }^{\gamma}$ is $\gamma$-traceless on $c \gamma$. Since $\mathcal{R}_{\alpha[c a b]}=0$, we conclude that $\mathcal{R}_{\alpha c a b}$ must be in the irreducible hook representation in $c \mid a b$. However, we 
have already fixed that piece to vanish using $H_{\alpha c \mid a b}$ in (4.70b). Thus we conclude that $\mathcal{R}_{\alpha \beta c}{ }^{\gamma}$ and $\mathcal{R}_{\alpha c a b}$ both vanish entirely. The remaining Bianchi identities immediately give

$$
\begin{aligned}
\mathcal{B}_{\alpha b}=0 & \Longrightarrow & \mathcal{T}^{\alpha}=0, \\
\mathcal{B}_{\alpha \bar{b}}=0 & \Longrightarrow & \mathcal{R}_{\alpha \bar{b} \overline{c d}} \eta^{\overline{b d}}=0 \quad \Longrightarrow \quad \mathcal{R}_{\alpha \bar{b} \overline{c d}}=0 .
\end{aligned}
$$

The first equation is the covariantized dilatino equation of motion. The last implication in the second equation follows because (with $\mathcal{R}_{\alpha[\bar{b} \bar{c}]}$ eliminated) only the irreducible hook representation remains, but this is precisely the piece we can remove by choosing $H_{\alpha \bar{b} \mid \overline{c d}}$ as in (4.70a). In this way we have eliminated all dimension $\frac{3}{2}$ components of torsion and curvature.

Finally, we turn to the dimension 2 Bianchi identities. First, we analyze the purely bosonic pieces of (4.71a):

$$
\begin{array}{lll}
\mathcal{B}_{\overline{a b c d}}=0 & \Longrightarrow & \mathcal{R}_{[\overline{a b} \bar{c}]}=0, \\
\mathcal{B}_{a b c d}=0 & \Longrightarrow & \mathcal{R}_{[a b c d]}=0, \\
\mathcal{B}_{a \overline{b c d}}=0 & \Longrightarrow & \mathcal{R}_{a[\bar{b} \bar{c}]}=0, \\
\mathcal{B}_{a b c \bar{d}}=0 & \Longrightarrow & \mathcal{R}_{\bar{d}[c a b]}=0, \\
\mathcal{B}_{a b \overline{c d}}=0 & \Longrightarrow & \mathcal{R}_{a b \overline{c d}}=-\mathcal{R}_{\overline{c d} a b} .
\end{array}
$$

The first and second equations work similarly. Together with the conventional constraints (4.70f) and (4.70g), they imply that $\mathcal{R}_{a b c d}$ and $\mathcal{R}_{\overline{a b}} \overline{c d}$ are pure traces. The third and fourth equations, together with (4.70c) and (4.70d) imply that $\mathcal{R}_{a \bar{b} c d}$ and $\mathcal{R}_{a \bar{b}} \overline{c d}$ each consist of only the $\square \times \bar{\square}$ representations. Finally the last equation, coupled with (4.70e) tells us that $\mathcal{R}_{a b \overline{c d}}$ vanishes. In summary, we have

$$
\begin{array}{ll}
\mathcal{R}_{a b c d}=\frac{1}{45} \eta_{a[c} \eta_{d] b} \mathcal{R} & \mathcal{R}_{\overline{a b} \overline{c d}}=\frac{1}{45} \eta_{\bar{a}[\bar{c}} \eta_{\bar{d}] \bar{b}} \overline{\mathcal{R}} \\
\mathcal{R}_{a \bar{b} c d}=\frac{2}{9} \eta_{a[c} \mathcal{R}_{\bar{b} e d]}^{e}, & \mathcal{R}_{a \bar{b} \overline{c d}}=\frac{2}{9} \eta_{\bar{b}[\bar{d}} \mathcal{R}_{a \bar{e} \bar{c}]} \bar{e}, \\
\mathcal{R}_{a b \overline{c d}}=\mathcal{R}_{\overline{a b} c d}=0 . &
\end{array}
$$

The remaining Bianchi identities from (4.71a) (and using (4.70h)) are

$$
\begin{aligned}
& \mathcal{B}_{\alpha}{ }^{\beta} \overline{c d}=0 \quad \Longrightarrow \quad \mathcal{R}_{\alpha}{ }^{\beta} \overline{c d}=-\frac{1}{4} \mathcal{R}_{\overline{c d} a b}\left(\gamma^{a b}\right)_{\alpha}{ }^{\beta}=0 \\
& \mathcal{B}_{\alpha}{ }^{\beta} c d=0 \quad \Longrightarrow \quad \mathcal{R}_{\alpha}{ }^{\beta} c d=-\frac{1}{4}\left(\gamma^{a b}\right)_{\alpha}{ }^{\beta} \mathcal{R}_{c d a b}=-\frac{1}{180}\left(\gamma_{c d}\right)_{\alpha}{ }^{\beta} \mathcal{R}, \\
& \mathcal{B}_{\alpha}{ }^{\beta} \gamma^{\delta}=0 \quad \Longrightarrow \mathcal{R}_{\alpha \gamma}{ }^{\beta \delta}=-\mathcal{R}_{(\alpha}{ }^{(\beta}{ }_{c d}\left(\gamma^{c d}\right)_{\gamma)}{ }^{\delta)}=\frac{1}{180}\left(\gamma^{c d}\right)_{(\alpha}{ }^{(\beta}\left(\gamma_{c d}\right)_{\gamma)}{ }^{\delta)} \mathcal{R}, \\
& \mathcal{B}_{\alpha}{ }^{\beta}{ }_{c \bar{d}}=0 \quad \Longrightarrow \quad \mathcal{R}_{\alpha \bar{d} c}{ }^{\beta}=\frac{1}{4}\left(\gamma^{a b}\right)_{\alpha}{ }^{\beta} \mathcal{R}_{c \bar{d} a b} .
\end{aligned}
$$

The last equation above implies a constraint on $\mathcal{R}_{c \bar{d} a b}$ due to the $\gamma$-traceless left-hand side, but this kills the remaining representation, leading to

$$
\mathcal{R}_{a \bar{b} c d}=0, \quad \mathcal{R}_{\alpha \bar{b} c}^{\gamma}=0
$$


We are nearly finished. The remaining Bianchi identities at dimension two are

$$
\begin{aligned}
& \mathcal{B}_{a \bar{b}}=0 \quad \Longrightarrow \quad \mathcal{R}_{a \bar{d} \bar{b}}{ }^{\bar{d}}=-\mathcal{R}_{\bar{b} d a}{ }^{d}, \\
& \mathcal{B}_{a b}=0 \quad \Longrightarrow \quad \mathcal{R}_{a b}=0 \text {, } \\
& \mathcal{B}_{\overline{a b}}=0 \quad \Longrightarrow \quad \mathcal{R}_{\overline{a b}}=0, \\
& \mathcal{B}=0 \quad \Longrightarrow \quad \mathcal{R}+\overline{\mathcal{R}}=0 \text {, } \\
& \mathcal{B}_{\alpha}{ }^{\beta}=0 \quad \Longrightarrow \quad \mathcal{R}_{\alpha \gamma}{ }^{\gamma \beta}=0 \quad \Longrightarrow \quad \mathcal{R}=0 \text {. }
\end{aligned}
$$

The first four equations are analogous to bosonic ones. The first tells us that there is a single "Einstein tensor" given by $\mathcal{R}_{a \bar{b}}=\mathcal{R}_{a \bar{d} \bar{b}}{ }^{\bar{d}}=-\mathcal{R}_{\bar{b} d a}{ }^{d}$, but this vanishes due to the $\mathcal{B}_{\alpha}{ }^{\beta} \overline{c d}$ Bianchi discussed above. The fourth equation tells us that there is a single scalar curvature, given by $\mathcal{R}=\mathcal{R}_{a b}{ }^{a b}=-\mathcal{R}_{\overline{a b}} \overline{a b}$. The final Bianchi identity tells us this scalar curvature vanishes as well.

Remarkably, all of the torsions and curvatures have been eliminated through dimension two. This was to be expected because there are no on-shell component curvatures or covariant fields at this level.

\section{Derivation of component supersymmetric DFT}

The analysis by Siegel [2] did not directly address the component structure of double field theory. Since then, the component supersymmetric formulation has been independently derived by Hohm and Kwak [12] to second order in fermions and by Jeon, Lee, and Park to all orders [13]. Already one can see from the linearized transformations (3.1)-(3.3) that the results of $[12,13]$ are recovered to this order if we solve the fermionic part of the section condition by setting $\partial^{\mu}=0$, leaving the doubled bosonic space untouched. Our goal in the remainder of this paper will be to flesh out this connection to all orders.

It may be surprising that this is not as easy as one might expect. There are standard techniques developed for relating component fields to superfields, and in the case where the superfield description is geometric - that is, involving connections, curvatures, gauge transformations, diffeomorphisms, and so on - the path from superspace to components is straightforward. Much of that intuition implicitly assumes that diffeomorphisms and the supervielbein have a simple GL $(D \mid s)$ structure. Since that no longer is the case here, we will find that greater care must be taken.

\subsection{Conventional $\mathcal{N}=1$ superspace: a review and a redo}

As a brief review and a warm-up, let's recall how conventional superspace leads to a component theory. We take a conventional $\mathcal{N}=1$ superspace for some $\operatorname{GL}(D \mid s)$ structure group. The supervielbein is

$$
E_{\mathrm{M}}^{\mathrm{A}}=\left(\begin{array}{cc}
e_{\mathrm{m}}^{\mathrm{a}} & \psi_{\mathrm{m}}{ }^{\alpha} \\
E_{\mu}{ }^{\mathrm{a}} & E_{\mu}{ }^{\alpha}
\end{array}\right) .
$$

The fields denoted $e_{\mathrm{m}}{ }^{\mathrm{a}}$ and $\psi_{\mathrm{m}}{ }^{\alpha}$ correspond to the physical vielbein and gravitino; the other two fields are unphysical. To see that $e$ and $\psi$ transform appropriately, recall that 
superdiffeomorphisms act as

$$
\delta_{\xi} E_{\mathrm{M}}^{\mathrm{A}}=\xi^{\mathrm{N}} \partial_{\mathrm{N}} E_{\mathrm{M}}^{\mathrm{A}}+\partial_{\mathrm{M}} \xi^{\mathrm{N}} E_{\mathrm{N}}^{\mathrm{A}}
$$

Specializing to a purely bosonic superdiffeomorphism, $\xi^{\mathrm{M}}=\left(\xi^{\mathrm{m}}, 0\right)$, it is easy to see that $e$ and $\psi$ transform as conventional 1-forms with parameter $\xi^{\mathrm{m}}$. Considering now covariant superdiffeomorphisms

$$
\delta_{\xi}^{\text {cov }} E_{\mathrm{M}}^{\mathrm{A}}=\mathcal{D}_{\mathrm{M}} \xi^{\mathrm{A}}+E_{\mathrm{M}}{ }^{\mathrm{B}} \xi^{\mathrm{C}} T_{\mathrm{CB}}{ }^{\mathrm{A}}
$$

one can see that specializing to a fermionic parameter $\xi^{\mathrm{A}}=\left(0, \epsilon^{\alpha}\right)$ gives $^{11}$

$$
\begin{aligned}
\delta e_{\mathrm{m}}{ }^{\mathrm{a}} & =e_{\mathrm{m}}{ }^{\mathrm{b}} \epsilon^{\gamma} T_{\gamma \mathrm{b}}{ }^{\mathrm{a}}+\psi_{\mathrm{m}}{ }^{\beta} \epsilon^{\gamma} T_{\gamma \beta}{ }^{\mathrm{a}}, \\
\delta \psi_{\mathrm{m}}{ }^{\alpha} & =\mathcal{D}_{\mathrm{m}} \epsilon^{\alpha}+e_{\mathrm{m}}{ }^{\mathrm{b}} \epsilon^{\gamma} T_{\gamma \mathrm{b}}{ }^{\alpha}+\psi_{\mathrm{m}}{ }^{\beta} \epsilon^{\gamma} T_{\gamma \beta}{ }^{\alpha} .
\end{aligned}
$$

For the vielbein, the first torsion term usually vanishes and the second is a $\gamma$-matrix. For the gravitino, its torsion terms usually involve additional fields present in the supergravity multiplet. These transformations involve no explicit $\theta$ derivatives and can consistently be applied at $\theta=0$. The additional fields $E_{\mu}{ }^{a}$ and $E_{\mu}{ }^{\alpha}$ can be set to zero at lowest order in $\theta$ as they transform with leading terms $\mathcal{D}_{\mu} \xi^{\mathrm{a}}$ and $\mathcal{D}_{\mu} \xi^{\alpha}$. By a judicious choice of the higher $\theta$-dependence of $\xi^{\mathrm{a}}$ and $\xi^{\alpha}$ (which do not affect the bottom components of $e_{\mathrm{m}}{ }^{\mathrm{a}}$ and $\psi_{\mathrm{m}}{ }^{\alpha}$ ), one can eliminate the bottom components of $E_{\mu}{ }^{a}$ and $E_{\mu}{ }^{\alpha} \cdot{ }^{12}$

A parallel analysis as above is not really available in double field theory. The main hangup is that the supervielbein in DFT is a constrained object: its entries are not independent. Let us redo the above analysis in a way that will generalize more directly to the DFT situation. Admittedly, this will look rather pointlessly complicated for $\operatorname{GL}(D \mid s)$.

We begin by decomposing the supervielbein as

$$
E_{\mathrm{M}}^{\mathrm{A}}=\left(\begin{array}{cc}
e_{\mathrm{m}}{ }^{\mathrm{a}} & \psi_{\mathrm{m}}{ }^{\alpha} \\
\Xi_{\mu}{ }^{\mathrm{n}} e_{\mathrm{n}}{ }^{\mathrm{a}} & \phi_{\mu}{ }^{\alpha}+\Xi_{\mu}{ }^{\mathrm{n}} \psi_{\mathrm{n}}{ }^{\alpha}
\end{array}\right)
$$

where $\Xi$ and $\phi$ are new parametrizations for the second row. Denoting the tangent-space valued gravitino with a tilde for compactness, $\tilde{\psi}_{\mathrm{a}}{ }^{\alpha}=e_{\mathrm{a}}{ }^{\mathrm{m}} \psi_{\mathrm{m}}{ }^{\alpha}$, this expression can be written as the product of four factors:

$$
\begin{aligned}
E_{\mathrm{M}}{ }^{\mathrm{A}} & =\left(\begin{array}{ll}
1 & 0 \\
\Xi & 1
\end{array}\right) \times\left(\begin{array}{ll}
1 & 0 \\
0 & \phi
\end{array}\right) \times\left(\begin{array}{ll}
e & 0 \\
0 & 1
\end{array}\right) \times\left(\begin{array}{ll}
1 & \tilde{\psi} \\
0 & 1
\end{array}\right) \\
& =\exp \left(\Xi_{\mu}{ }^{\mathrm{m}} X_{\mathrm{m}}{ }^{\mu}\right) \times \exp \left(a_{\mu}{ }^{\nu} X_{\nu}{ }^{\mu}\right) \times \exp \left(a_{\mathrm{m}}{ }^{\mathrm{n}} X_{\mathrm{n}}{ }^{\mathrm{m}}\right) \times \exp \left(\tilde{\psi}_{\mathrm{m}}{ }^{\mu} X_{\mu}{ }^{\mathrm{m}}\right) \\
& =\mathcal{V}_{-1} \times \mathcal{V}_{0^{\prime}} \times \mathcal{V}_{0} \times \mathcal{V}_{+1}
\end{aligned}
$$

\footnotetext{
${ }^{11}$ The two classes of transformations, $\xi^{\mathrm{M}}=\left(\xi^{\mathrm{m}}, 0\right)$ and $\xi^{\mathrm{A}}=\left(0, \epsilon^{\alpha}\right)$, span the full space of transformations, so we lose nothing by focusing on these.

${ }^{12}$ This is not quite the whole story because one should inquire about the higher $\theta$-components of these superfields. One can show using a normal coordinate expansion in $\theta$, that the $\theta$-expansion of $E_{\mathrm{M}}{ }^{\mathrm{A}}$ is determined purely from the bottom components of $E_{\mathrm{M}}{ }^{\mathrm{A}}$, the torsion tensor, the Riemann tensor, and their various covariant derivatives.
} 
We have decomposed the $\mathrm{GL}(D \mid s)$ generators $X_{\mathrm{M}}{ }^{\mathrm{N}}$ as $X_{\mathrm{m}}{ }^{\nu}, X_{\mu}{ }^{\nu}, X_{\mathrm{m}}{ }^{\mathrm{n}}$, and $X_{\mu}{ }^{\mathrm{n}}$. An external automorphism of the algebra permits us to assign level -1 to the first generator, 0 to the second and third, and +1 to the last. We can label the three factors as $\mathcal{V}_{\ell}$ with respect to this level. In the above parametrization, $e=\exp \left(a_{\mathrm{m}}{ }^{\mathrm{n}}\right)$ and $\phi=\exp \left(a_{\mu}{ }^{\nu}\right)$, and we will work with $e$ and $\phi$ directly.

Let's check that this makes sense. A Lorentz transformation acts on the right as $\delta E=-E \Lambda$ and is purely level $0\left(\right.$ and $\left.0^{\prime}\right)$. It follows that

$$
\delta_{\Lambda} \mathcal{V}_{+1}=-\left[\mathcal{V}_{+1}, \Lambda\right], \quad \delta_{\Lambda}\left(\mathcal{V}_{0^{\prime}} \mathcal{V}_{0}\right)=\mathcal{V}_{0^{\prime}} \mathcal{V}_{0} \Lambda, \quad \delta_{\Lambda} \mathcal{V}_{-1}=0
$$

In other words, the gravitino $\tilde{\psi}$ is covariant on both indices, $e$ and $\phi$ transform only from the right, and $\Xi$ is invariant.

Next, we check the action of diffeomorphisms. For simplicity, we ignore the transport term, considering only general coordinate transformations, where $\delta_{\text {g.c. }} E=\mathcal{K} E$ for $\mathcal{K}=$ $\partial_{M} \xi^{N}$. This has an obvious level decomposition:

$$
\mathcal{K}_{-1}=\partial_{\mu} \xi^{n}, \quad \mathcal{K}_{0}=\partial_{m} \xi^{n}, \quad \mathcal{K}_{0^{\prime}}=\partial_{\mu} \xi^{\nu}, \quad \mathcal{K}_{+1}=\partial_{m} \xi^{\nu}
$$

We focus on bosonic transformations with $\xi^{\mathrm{M}}=\left(\xi^{\mathrm{m}}, 0\right)$. Then only $\mathcal{K}_{-1}$ and $\mathcal{K}_{0}$ are nonzero, leading to

$$
\delta_{\text {g.c. }} \mathcal{V}_{-1}=\mathcal{K}_{-1}+\left[\mathcal{K}_{0}, \mathcal{V}_{-1}\right], \quad \delta_{\text {g.c. }} \mathcal{V}_{0}=\mathcal{K}_{0} \mathcal{V}_{0}, \quad \delta_{\text {g.c. }} \mathcal{V}_{0^{\prime}}=0, \quad \delta_{\text {g.c. }} \mathcal{V}_{+1}=0
$$

Evidently, $\Xi$ shifts by $\partial_{\mu} \xi^{\text {n }}$ (so its lowest component can be set to zero), $e$ transforms as a 1-form, and $\phi$ and $\tilde{\psi}$ are scalars.

Finally, we look at covariant diffeomorphisms, which are given by

$$
\delta E=E \mathcal{K}, \quad \mathcal{K}_{\mathrm{B}}{ }^{\mathrm{A}}=\mathcal{D}_{\mathrm{B}} \xi^{\mathrm{A}}+\xi^{\mathrm{C}} T_{\mathrm{CB}}{ }^{\mathrm{A}} .
$$

We are interested in a fermionic transformation with $\xi^{\mathrm{A}}=\left(0, \epsilon^{\alpha}\right)$. The first term of $\mathcal{K}$ contributes only to $\mathcal{K}_{\mathrm{b}}{ }^{\alpha}$ and $\mathcal{K}_{\beta}{ }^{\alpha}$, but the torsion term contributes to all elements of $\mathcal{K}$, at least in principle. Now we need to equate this to an arbitrary variation of $E$. This can be written as $\delta E=E \mathcal{J}$, but it's actually going to be more useful to parametrize the current a little differently. We choose to define $\dot{\mathcal{J}}$ via

$$
\delta E=\mathcal{V}_{-1} \mathcal{V}_{0^{\prime}} \mathcal{V}_{0} \times \stackrel{\mathcal{J}}{ } \times \mathcal{V}_{+1}
$$

This is related to $\mathcal{J}=E^{-1} \delta E$ by composition by $\mathcal{V}_{+1}, \stackrel{\circ}{\mathcal{J}}=\mathcal{V}_{+1} \mathcal{J}\left(\mathcal{V}_{+1}\right)^{-1}$.

At this point, decomposing the vielbein with respect to the level $\ell$ starts to become notationally cluttered. Let's denote $\mathcal{V}_{\Xi}=\mathcal{V}_{-1} \mathcal{V}_{0^{\prime}}$ and $\mathcal{V}_{\Psi}=\mathcal{V}_{+1}$, so that

$$
E=\mathcal{V}_{\Xi} \times \mathcal{V}_{0} \times \mathcal{V}_{\Psi}
$$

The pieces $\mathcal{V}_{0}$ and $\mathcal{V}_{\Psi}$ involve the component graviton and gravitino, while $\mathcal{V}_{\Xi}$ involves pieces that can be set to zero at lowest level in $\theta$. Then we have

$$
\delta E=\mathcal{V}_{\Xi} \mathcal{V}_{0} \times \stackrel{\mathcal{J}}{ } \times \mathcal{V}_{\Psi}, \quad \stackrel{\circ}{\mathcal{J}}=\mathcal{V}_{\Psi} \mathcal{J} \mathcal{V}_{\Psi}^{-1}
$$


The current $\mathcal{J}$ decomposes as follows:

$$
\begin{array}{ll}
\dot{\mathcal{J}}_{\mathrm{a}}^{\mathrm{b}} \equiv \Delta e_{\mathrm{a}}{ }^{\mathrm{b}}=e_{\mathrm{a}}{ }^{\mathrm{m}} \delta e_{\mathrm{m}}{ }^{\mathrm{a}}, & \mathcal{J}_{\mathrm{a}}{ }^{\beta}=\delta \tilde{\psi}_{\mathrm{a}}{ }^{\beta}=\delta\left(e_{\mathrm{a}}{ }^{\mathrm{m}} \psi_{\mathrm{m}}{ }^{\beta}\right), \\
\mathcal{J}_{\alpha}{ }^{\beta}=\phi_{\alpha}{ }^{\mu} \delta \phi_{\mu}{ }^{\beta} & \mathcal{J}_{\alpha}{ }^{\mathrm{b}}=\phi_{\alpha}{ }^{\mu} \delta \Xi_{\mu}{ }^{\mathrm{n}} e_{\mathrm{n}}{ }^{\mathrm{a}} .
\end{array}
$$

We want to equate this with $\stackrel{\mathcal{K}}{=}=\mathcal{V}_{\Psi} \mathcal{K}\left(\mathcal{V}_{\Psi}\right)^{-1}$. Writing $\mathcal{V}_{\Psi}=\exp \psi$ in terms of the level +1 generator $\psi$, we can compute easily that

$$
\begin{aligned}
\stackrel{\circ}{\mathcal{K}}+1_{+1} & =\mathcal{K}_{+1}+\left[\psi, \mathcal{K}_{0}+\mathcal{K}_{0^{\prime}}\right]+\frac{1}{2}\left[\psi,\left[\psi, \mathcal{K}_{-1}\right]\right] \\
\dot{\mathcal{K}}_{0}+\dot{\mathcal{K}}_{0^{\prime}} & =\mathcal{K}_{0}+\mathcal{K}_{0^{\prime}}+\left[\psi, \mathcal{K}_{-1}\right] \\
\dot{\mathcal{K}}_{-1} & =\mathcal{K}_{-1} .
\end{aligned}
$$

From this, one can read off the graviton variation,

$$
\Delta e_{\mathrm{a}}^{\mathrm{b}}=e_{\mathrm{a}}{ }^{\mathrm{m}} \delta e_{\mathrm{m}}^{\mathrm{b}}=\epsilon^{\gamma} T_{\gamma \mathrm{a}}^{\mathrm{b}}+\psi_{\mathrm{a}}{ }^{\alpha} \epsilon^{\gamma} T_{\gamma \alpha}^{\mathrm{b}}
$$

which coincides with (5.4). For the gravitino, the answer is more involved:

$$
\begin{aligned}
\delta\left(e_{\mathrm{a}}{ }^{\mathrm{m}} \psi_{\mathrm{m}}{ }^{\beta}\right) & =\mathcal{D}_{\mathrm{a}} \epsilon^{\beta}+\epsilon^{\gamma} T_{\gamma \mathrm{a}}{ }^{\beta}+\psi_{\mathrm{a}}{ }^{\alpha}\left(\mathcal{D}_{\alpha} \epsilon^{\beta}+\epsilon^{\gamma} T_{\gamma \alpha}{ }^{\beta}\right)-\epsilon^{\gamma} T_{\gamma \mathrm{a}}{ }^{\mathrm{b}} \psi_{\mathrm{b}}{ }^{\beta}-\psi_{\mathrm{a}}{ }^{\alpha} \epsilon^{\gamma} T_{\gamma \alpha}{ }^{\mathrm{b}} \psi_{\mathrm{b}}{ }^{\beta} \\
& =e_{\mathrm{a}}{ }^{\mathrm{m}} \mathcal{D}_{\mathrm{m}} \epsilon^{\beta}+\epsilon^{\gamma} T_{\gamma \mathrm{a}}{ }^{\beta}+\psi_{\mathrm{a}}{ }^{\alpha} \epsilon^{\gamma} T_{\gamma \alpha}{ }^{\beta}-\Delta e_{\mathrm{a}}{ }^{\mathrm{b}} \psi_{\mathrm{b}}{ }^{\beta}
\end{aligned}
$$

The two covariant derivative terms have combined into $e_{\mathrm{a}}{ }^{\mathrm{m}} \mathcal{D}_{\mathrm{m}}$, which is independent of $\theta$ derivatives. The first three terms in the final expression coincide with the expected result (5.4), and the last arises just from the transformation of the vielbein. Finally, one can also show that the leading contribution to $\Delta \phi_{0^{\prime}}$ is as before $\partial_{\mu} \xi^{\alpha}$, so this term can be eliminated to lowest order in $\theta$.

\subsection{Level decomposition of the doubled supervielbein}

In parallel with the analysis of the conventional $\mathcal{N}=1$ supervielbein, we will first perform a level decomposition of the generators $X^{\mathcal{M N}}$ of $\operatorname{OSp}(10,10 \mid 32)$ :

$$
\underbrace{X^{\mu \nu}}_{\text {level -2 }}, \underbrace{X^{\mu \hat{n}}}_{\text {level -1 }}, \underbrace{X^{\hat{m} \hat{n}}, X_{\mu}^{\nu}}_{\text {level } 0}, \underbrace{X_{\mu \hat{m}}}_{\text {level +1 }}, \underbrace{X_{\mu \nu}}_{\text {level }+2},
$$

where the level denotes the difference between the number of lower and upper fermionic indices. As in the previous example, we are going to place the positive level generators to the right in $\mathcal{V}_{\mathcal{M}}{ }^{\mathcal{A}}$, so they are naturally going to lead to fields possessing tangent space indices. With that in mind, we introduce fields associated with these generators in table 4 .

The fields associated to the positive level generators we have denoted $C^{\alpha \beta}, \chi_{a}{ }^{\alpha}$, and $\Psi_{\bar{a}}{ }^{\alpha}$. Using the additional tangent space symmetry, we are going to gauge away $C^{\alpha \beta}$ and the spin-3/2 part of $\chi_{a}{ }^{\alpha}$, fixing the latter to $\chi_{a}{ }^{\alpha}=\frac{1}{10}\left(\gamma_{a}\right)^{\alpha \beta} \chi_{\beta}$. The field $V_{\hat{m}}{ }^{\hat{a}}$ will become the bosonic doubled vielbein. The remaining fields have analogues with fields in conventional $\mathcal{N}=1$ superspace. $\phi_{\mu}{ }^{\alpha}$ is the identically named field there, and corresponds to the inverse of the $\mathcal{N}=1$ superspace vielbein component $E_{\alpha}{ }^{\mu} . \Xi_{\mu}{ }^{\mathrm{n}}$ corresponds to the similarly named field there. The remaining fields $\Xi_{\mu \mathrm{n}}$ and $B_{\mu \nu}$ correspond to the fermionic legs of the super 


\begin{tabular}{ccc}
\hline field & generator & level \\
\hline$C^{\alpha \beta}$ & $X_{\alpha \beta}$ & +2 \\
$\chi_{a}{ }^{\beta}$ & $X_{\beta}{ }^{a}$ & +1 \\
$\Psi_{\bar{a}}{ }^{\beta}$ & $X_{\beta}{ }^{\bar{a}}$ & +1 \\
$V_{\hat{m}}{ }^{\hat{a}}$ & $X_{\hat{m}}{ }^{\hat{n}}$ & 0 \\
$\phi_{\mu}{ }^{\alpha}$ & $X_{\mu}{ }^{\nu}$ & 0 \\
$\Xi_{\mu}{ }^{\hat{m}}$ & $X_{\hat{m}}{ }^{\mu}$ & -1 \\
$B_{\mu \nu}$ & $X^{\mu \nu}$ & -2 \\
\hline
\end{tabular}

Table 4. Constituent fields of the OSp supervielbein. Positive level fields are written with Lorentz indices.

2 -form $B_{\mathrm{MN}}$. As in conventional $\mathcal{N}=1$ superspace, the bottom components of $\phi_{\mu}{ }^{\alpha}, \Xi_{\mu}{ }^{\mathrm{n}}$, and $B_{\mu \nu}$ can be eliminated by using the higher $\theta$-components of superdiffeomorphisms.

We want to decompose the supervielbein analogously to how we did in conventional superspace (5.6). To fix our conventions, we normalize the fields associated with the nonzero generators so that they fill out a graded symmetric element $\mathcal{A}_{\mathcal{A B}}$ of $\operatorname{OSp}(10,10 \mid 32)$ as

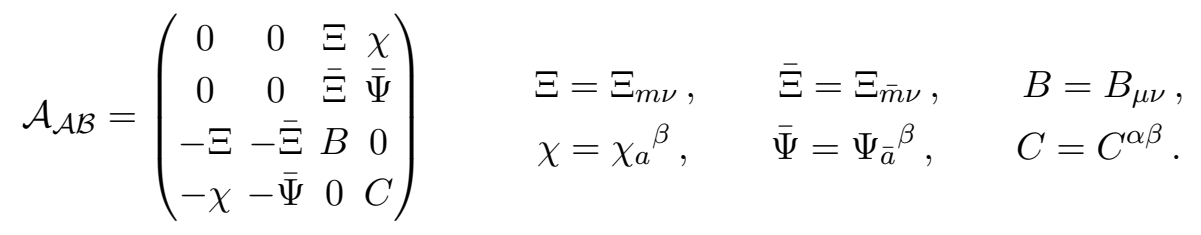

We will be using the left/right basis for the generators, so that $\Xi_{\hat{m} \nu}$ has been decomposed into $\Xi_{m \nu}$ and $\Xi_{\bar{m} \nu}$. We have also distinguished between world and tangent space indices based on how we are going to place these generators into the coset, although this is perhaps sloppy notation.

We have denoted the gravitino by $\bar{\Psi}$ above because later on it will be convenient to also use $\Psi=\Psi_{a}{ }^{\beta}$ as an alias for $\chi_{a}{ }^{\beta}$. Then $\Psi$ and $\bar{\Psi}$ appear in an analogous way as $\Xi$ and $\bar{\Xi}$ in some of the formulae. We won't do this just yet because we want to keep in mind $\chi$ is the dilatino and not a second gravitino.

Exponentiating the above generators using $\mathcal{V}=\exp \left(\mathcal{A}_{\mathcal{A}}{ }^{\mathcal{B}}\right)$ for each level gives

$$
\begin{aligned}
& \mathcal{V}_{+2}=\left(\begin{array}{llll}
1 & 0 & 0 & 0 \\
0 & 1 & 0 & 0 \\
0 & 0 & 1 & 0 \\
0 & 0 & C & 1
\end{array}\right), \quad \mathcal{V}_{-2}=\left(\begin{array}{cccc}
1 & 0 & 0 & 0 \\
0 & 1 & 0 & 0 \\
0 & 0 & 1 & -B \\
0 & 0 & 0 & 1
\end{array}\right), \quad B=B_{\mu \nu}, \quad C=C^{\alpha \beta}, \\
& \mathcal{V}_{+1}=\left(\begin{array}{cccc}
1 & 0 & \chi & 0 \\
0 & 1 & \bar{\Psi} & 0 \\
0 & 0 & 1 & 0 \\
-\chi^{T} & \bar{\Psi}^{T}-\frac{1}{2}\left(\chi^{T} \chi-\bar{\Psi}^{T} \bar{\Psi}\right) & 1
\end{array}\right), \quad \begin{array}{c}
\chi=\chi_{a}^{\beta}, \quad \\
\left(\chi^{T}\right)^{\alpha b}=\chi^{b \alpha}, \quad\left(\bar{\Psi}^{T}\right)^{\alpha \bar{b}}=\bar{\Psi}^{\beta} \bar{\Psi}^{\bar{b} \alpha},
\end{array},
\end{aligned}
$$




$$
\mathcal{V}_{-1}=\left(\begin{array}{cccc}
1 & 0 & 0 & -\Xi \\
0 & 1 & 0 & -\overline{\bar{\Xi}} \\
-\Xi^{T} & \bar{\Xi}^{T} & 1 & \frac{1}{2}\left(\Xi^{T} \Xi-\bar{\Xi}^{T} \bar{\Xi}\right) \\
0 & 0 & 0 & 1
\end{array}\right), \quad \begin{aligned}
& \Xi=\Xi_{m \nu}, \\
& \left(\Xi^{T}\right)_{\mu}{ }^{n}=\Xi_{\mu}^{n}, \quad\left(\bar{\Xi}^{T}\right)_{\mu}{ }^{\bar{n}}=\bar{\Xi}_{\bar{m} \nu}{ }_{\mu}
\end{aligned}
$$

At level 0 , there are two commuting generators $X_{\hat{m}}^{\hat{n}}$ and $X_{\mu}{ }^{\nu}$. Their exponentiated elements are

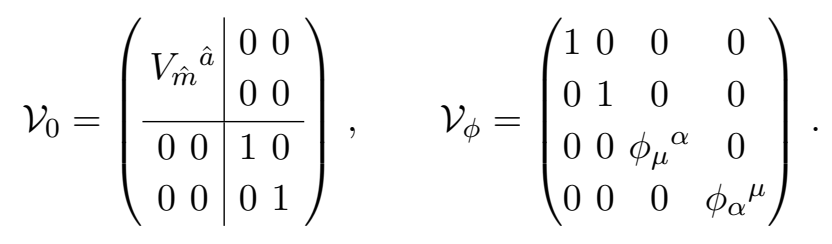

Here $V_{\hat{m}}{ }^{\hat{a}}$ will become the component DFT vielbein.

Using these building blocks, we can construct a generic orthosymplectic element as

$$
\mathcal{V}=\underbrace{\mathcal{V}_{-2} \mathcal{V}_{-1} \mathcal{V}_{\phi}}_{\mathcal{V}_{\Xi}} \times \mathcal{V}_{0} \times \underbrace{\mathcal{V}_{+1} \mathcal{V}_{+2}}_{\mathcal{V}_{\Psi}}=\mathcal{V}_{\Xi} \times \mathcal{V}_{0} \times \mathcal{V}_{\Psi}
$$

The interpretation of these three factors will be similar to conventional $\mathcal{N}=1$ superspace. The factor $\mathcal{V}_{0}$ contains the bosonic double vielbein. $\mathcal{V}_{\Psi}$ involves the gravitino, dilatino, and other pieces that will be set to zero by a tangent space transformation. $\mathcal{V}_{\Xi}$ involves fields that live purely in superspace and have no component analogues, as their $\theta=0$ parts can be eliminated by a $\theta$-dependent diffeomorphism.

Let's see now if the decomposition we have chosen is actually sensible from the perspective of the component theory. Specifically, does it lead to a sensible parametrization of the derivatives $D_{\mathcal{A}}$ ? It is helpful to first split off the gravitino (and dilatino) piece and write $\stackrel{\mathcal{V}}{=} \mathcal{V}_{\Xi} \mathcal{V}_{0}$. This is given by

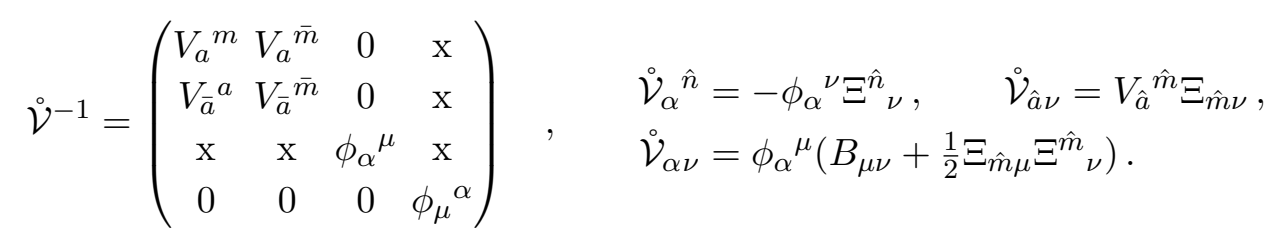

The entries marked $\mathrm{x}$ in the matrix are given above. As we will solve the fermionic part of the section condition by taking $\partial^{\mu}=0$, it follows that

$$
\stackrel{\circ}{D}_{\mathcal{A}}=\stackrel{\circ}{\mathcal{V}}_{\mathcal{A}}{ }^{\mathcal{M}} \partial_{\mathcal{M}}=\left(\begin{array}{c}
V_{\hat{a}}{ }^{\hat{m}} \partial_{\hat{m}} \\
\dot{\mathcal{V}}_{\alpha}{ }^{\hat{m}} \partial_{\hat{m}}+\phi_{\alpha}{ }^{\mu} \partial_{\mu} \\
0
\end{array}\right) \equiv\left(\begin{array}{c}
\stackrel{\circ}{D}_{\hat{a}} \\
\stackrel{D}{\alpha}_{\alpha} \\
0
\end{array}\right)
$$

where we define $\stackrel{\circ}{D}_{\hat{a}}$ and $\stackrel{\circ}{D}_{\alpha}$ by these expressions. Now we can build $D_{\mathcal{A}}=\mathcal{V}_{\mathcal{A}} \mathcal{M}_{\mathcal{M}}=$ $\left(\mathcal{V}_{\Psi}^{-1}\right)_{\mathcal{A}}^{\mathcal{B}} \stackrel{\circ}{\mathcal{B}}_{\mathcal{B}}$ and this leads to

$$
D_{\mathcal{A}}=\left(\mathcal{V}_{\Psi}^{-1}\right)_{\mathcal{A}} \stackrel{\mathcal{B}}{D}_{\mathcal{B}}=\left(\begin{array}{c}
\stackrel{\circ}{D}_{\hat{a}}-\Psi_{\hat{a}}^{\alpha} \stackrel{\circ}{D}_{\alpha} \\
\stackrel{\circ}{D}_{\alpha} \\
\Psi^{\hat{b} \alpha} \stackrel{\circ}{D}_{\hat{b}}-\left(C^{\alpha \beta}+\frac{1}{2} \Psi^{\hat{c} \alpha} \Psi_{\hat{c}}^{\beta}\right) \stackrel{\circ}{D}_{\beta}
\end{array}\right)
$$


where we have used the condensed notation $\Psi_{\hat{a}}^{\beta}=\left(\chi_{a}^{\beta}, \Psi_{\bar{a}^{\beta}}\right)$ and $\Psi^{\hat{a} \beta}=\left(\chi^{a \beta},-\Psi^{\bar{a} \beta}\right)$. It is extremely useful that $D_{\alpha}=\stackrel{\circ}{D}_{\alpha}$. We can then rewrite easily the other derivatives as

$$
D_{\hat{a}}=\stackrel{\circ}{D}_{\hat{a}}-\Psi_{\hat{a}}^{\beta} D_{\beta}, \quad D^{\alpha}=\Psi^{\hat{b} \alpha} D_{\hat{b}}-\left(C^{\alpha \beta}-\frac{1}{2} \Psi^{\hat{a} \alpha} \Psi_{\hat{a}}^{\beta}\right) D_{\beta} .
$$

The bosonic derivative $D_{\hat{a}}$ is now directly analogous to the conventional superspace derivative, where it is given by the component flat derivative $\stackrel{\circ}{D}_{\hat{a}}$ modified by a gravitino connection. The new operator in DFT is the dual fermionic derivative $D^{\alpha}$. Its explicit form will be crucial in subsequent computations. In neither expression above do the pieces of $\mathcal{V}_{\Xi}$ appear explicitly; they appear implicitly only via $D_{\alpha}$.

It is crucial that the above expressions hold only upon imposing $\partial^{\mu}=0$. Without this condition, the expressions are significantly more complicated.

\subsection{Generalized diffeomorphisms}

The next task is to figure out how the elements of the supervielbein transform, in order to verify that they have been correctly identified.

We begin with generalized diffeomorphisms. As in conventional superspace, it is best to split these up between standard diffeomorphisms $\xi^{\mathcal{M}}$ and covariant ones $\xi^{\mathcal{A}}$. It is not immediately obvious how to treat the dual fermionic direction, but it will turn out that we should keep this as a standard diffeomorphism. In other words, we are going to examine $\xi^{\mathcal{M}}=\left(\xi^{\hat{m}}, 0,-\tilde{\xi}_{\mu}\right)$ and $\xi^{\mathcal{A}}=\left(0, \epsilon^{\alpha}, 0\right)$. We begin with the former. Ignoring the transport term,

$$
\delta_{\text {g.c. }} \mathcal{V}_{\mathcal{M}}{ }^{\mathcal{A}}=\mathcal{K}_{\mathcal{M}} \mathcal{N}^{\mathcal{V}} \mathcal{V}_{\mathcal{N}}^{\mathcal{A}}, \quad \mathcal{K}_{\mathcal{M}}{ }^{\mathcal{N}}=\partial_{\mathcal{M}} \xi^{\mathcal{N}}-\partial^{\mathcal{N}} \xi_{\mathcal{M}}(-)^{n m}
$$

Since we restrict to $\partial^{\mu}=0$, it turns out that we only have non-positive levels for $\mathcal{K}$. Using the fact that the -1 generators commute with the -2 generators, one can show that

$$
\delta_{\text {g.c. }} \mathcal{V}_{\Xi}=\mathcal{K}_{-2} \mathcal{V}_{\Xi}+\mathcal{K}_{-1} \mathcal{V}_{\Xi}+\left[\mathcal{K}_{0}, \mathcal{V}_{\Xi}\right], \quad \delta_{\text {g.c. }} \mathcal{V}_{0}=\mathcal{K}_{0} \mathcal{V}_{0}, \quad \delta_{\text {g.c. }} \mathcal{V}_{\Psi}=0
$$

It is useful here that the level zero element $\mathcal{K}_{0}$ only involves $\partial_{\hat{m}} \xi^{\hat{n}}$ and not $\partial_{\mu} \xi^{\nu}$ or $\partial^{\mu} \tilde{\xi}_{\nu}$, as this simplifies the transformation of $\mathcal{V}_{\Xi}$. Because the $\mathcal{K}_{0}$ piece is just the bosonic $\mathrm{O}(10,10)$ general coordinate transformation, $\mathcal{V}_{0}$ transforms as a DFT vielbein should, while the gravitino and dilatino are scalar fields. The negative level transformations are completely soaked up by the fields in $\mathcal{V}_{\Xi}$, which transform as

$$
\delta B_{\mu \nu}=\partial_{\mu} \tilde{\xi}_{\nu}+\partial_{\nu} \tilde{\xi}_{\mu}, \quad \delta \Xi_{\hat{m} \nu}=\partial_{\hat{m}} \tilde{\xi}_{\nu}-\partial_{\nu} \xi_{\hat{m}}
$$

As in conventional superspace, these fields can be set to zero at lowest level in $\theta$.

Because the fields in $\mathcal{V}_{\Psi}$ are inert under $\tilde{\xi}_{\mu}$ and transform as scalars under $\xi^{\hat{m}}$, their gauge-fixing is not disturbed. That is, if $C^{\alpha \beta}=0$, it stays zero. If $\chi_{a}{ }^{\alpha}$ is $\gamma$-traceless, it remains so. This means component diffeomorphisms do not need to be defined with a compensating tangent space transformation. This is important: it ensures that their algebra remains unchanged in going from superspace to components. 


\subsection{Supersymmetry transformations}

Supersymmetry transformations are encoded in covariant diffeomorphisms. Let's analyze these next. Including a compensating tangent space transformation $\lambda_{\mathcal{A}}{ }^{\mathcal{B}}$ (which will be necessary in this case), the supervielbein transforms as $\delta \mathcal{V}=\mathcal{V}(\mathcal{K}-\lambda)$ where

$$
\mathcal{K}_{\mathcal{A}}^{\mathcal{B}}=\nabla_{\mathcal{A}} \xi^{\mathcal{B}}-\nabla^{\mathcal{B}} \xi_{\mathcal{A}}(-1)^{a b}+\xi^{\mathcal{C}} \mathcal{T}_{\mathcal{C} \mathcal{A}}{ }^{\mathcal{B}}
$$

What are the nonzero pieces? While our focus is on $\xi^{\mathcal{A}}=\left(0, \epsilon^{\alpha}, 0\right)$, we must be careful about expressions like $\nabla_{\mathcal{A}} \xi^{\mathcal{B}}=D_{\mathcal{A}} \xi^{\mathcal{B}}-\Omega_{\mathcal{A}}{ }^{\mathcal{B C}} \xi_{\mathcal{C}}$, which might lead to an unexpected contribution when the $\Omega$ connection is not purely double Lorentz. Luckily, the $\Omega$ piece in our case involves just $\Omega_{\mathcal{A}}{ }_{\gamma} \epsilon^{\gamma}$; there is no pollution from $\Omega_{\mathcal{A}}{ }^{\beta \gamma}$ and $\Omega_{\mathcal{A} b}{ }^{\gamma}$ because we have set $\xi_{\alpha}$ and $\xi_{a}$ to zero. We replace $\nabla$ with $\mathcal{D}$ here to emphasize $\mathcal{D}$ carries only the double Lorentz connection. For the torsion term, only $\mathcal{T}_{\gamma \beta a}$ is non-vanishing. This means the only nonzero elements of $\mathcal{K}_{\mathcal{A}}{ }^{\mathcal{B}}$ are

$$
\begin{aligned}
& \mathcal{K}^{\alpha \beta}=2 \mathcal{D}^{(\alpha} \epsilon^{\beta)}, \quad \mathcal{K}_{a}{ }^{\beta}=\mathcal{D}_{a} \epsilon^{\beta}, \quad \mathcal{K}_{\bar{a}}{ }^{\beta}=\mathcal{D}_{\bar{a}} \epsilon^{\beta}, \quad \mathcal{K}_{\alpha}{ }^{\beta}=\mathcal{D}_{\alpha} \epsilon^{\beta}, \quad \mathcal{K}_{\alpha}{ }^{b}=\kappa\left(\gamma^{b}\right)_{\alpha \gamma} \epsilon^{\gamma}, \\
& \mathcal{K}^{\alpha b}=-\mathcal{D}^{b} \epsilon^{\alpha}, \quad \mathcal{K}^{\alpha \bar{b}}=\mathcal{D}^{\bar{b}} \epsilon^{\alpha}, \quad \mathcal{K}_{\beta}^{\alpha}=-\mathcal{D}_{\beta} \epsilon^{\alpha}, \quad \mathcal{K}_{a \beta}=\kappa\left(\gamma_{a}\right)_{\beta \gamma} \epsilon^{\gamma},
\end{aligned}
$$

corresponding to levels $+2,+1,+1,0$, and -1 . The possible compensating $\lambda$ transformations are at levels $+2,+1$ and 0 .

As in the conventional $\mathcal{N}=1$ superspace example, we can parametrize an arbitrary variation as

$$
\delta \mathcal{V}=\mathcal{V} \times \mathcal{J}=\mathcal{V}_{\Xi} \mathcal{V}_{0} \times \stackrel{\circ}{\mathcal{J}} \times \mathcal{V}_{\Psi} \quad \Longrightarrow \quad \stackrel{\circ}{\mathcal{J}}=\mathcal{V}_{\Psi} \mathcal{J} \mathcal{V}_{\Psi}^{-1}
$$

For the case of a supersymmetry transformation, $\mathcal{J}=\mathcal{K}-\lambda$. Now work out $\mathcal{J}$ level-by-level, using the fact that $\mathcal{V}_{\Psi} \equiv \exp \Psi$ for a generator $\Psi$ at level +1 only. (We now work in the gauge where $C=0$.)

$$
\begin{aligned}
\stackrel{\mathcal{J}}{+2}= & \mathcal{K}_{+2}+\left[\Psi, \mathcal{K}_{+1}\right]+\frac{1}{2 !}\left[\Psi,\left[\Psi, \mathcal{K}_{0}\right]\right]+\frac{1}{3 !}\left[\Psi,\left[\Psi,\left[\Psi, \mathcal{K}_{-1}\right]\right]\right] \\
& -\lambda_{+2}-\left[\Psi, \lambda_{+1}\right]-\frac{1}{2 !}\left[\Psi,\left[\Psi, \lambda_{0}\right]\right] \\
\stackrel{\mathcal{J}}{+1}_{+1}= & \mathcal{K}_{+1}+\left[\Psi, \mathcal{K}_{0}\right]+\frac{1}{2}\left[\Psi,\left[\Psi, \mathcal{K}_{-1}\right]\right]-\lambda_{+1}-\left[\Psi, \lambda_{0}\right] \\
\stackrel{\circ}{\mathcal{J}}_{0}= & \mathcal{K}_{0}+\left[\Psi, \mathcal{K}_{-1}\right]-\lambda_{0} \\
\dot{\mathcal{J}}_{-1}= & \mathcal{K}_{-1} \\
\stackrel{\circ}{\mathcal{J}}_{-2}= & 0 .
\end{aligned}
$$

From the explicit expressions for $\mathcal{V}_{\ell}$, we find for the non-negative levels,

$$
\begin{array}{ll}
\left(\dot{\mathcal{J}}_{2}\right)^{\alpha \beta}=-\Psi^{\hat{a}(\alpha} \delta \Psi_{\hat{a}}{ }^{\beta)}, & \left(\AA_{\mathcal{J}}\right)_{\hat{a}}{ }^{\beta}=\delta \Psi_{\hat{a}}{ }^{\beta}, \\
\left(\dot{\mathcal{J}}_{0}\right)_{\hat{a}}{ }^{\hat{b}}=V_{\hat{a}}{ }^{\hat{m}} \delta V_{\hat{m}}{ }^{\hat{b}} \equiv J_{\hat{a}}{ }^{\hat{b}}, & \left(\dot{\mathcal{J}}_{0}\right)_{\alpha}{ }^{\beta}=\phi_{\alpha}{ }^{\mu} \delta \phi_{\mu}{ }^{\beta} .
\end{array}
$$

The expression for $\stackrel{\mathcal{J}}{2}_{2}$ is just going to tell is what the compensating $\lambda_{+2}=\lambda^{\alpha \beta}$ transformation needs to be, but its explicit form is irrelevant. The transformation of $\phi_{\mu}{ }^{\alpha}$ isn't really relevant 
here either - it's going to involve a leading term $\partial_{\mu} \epsilon^{\alpha}$ indicating that at lowest order in $\theta$ we can set $\phi_{\mu}{ }^{\alpha}=\delta_{\mu}{ }^{\alpha}$. For the bosonic DFT vielbein, we find that

$$
J_{a \bar{b}}=\kappa\left(\epsilon \gamma_{a} \Psi_{\bar{b}}\right), \quad J_{a b}=\frac{1}{5} \kappa\left(\epsilon \gamma_{a b} \chi\right)-\lambda_{a b}, \quad J_{\overline{a b}}=-\lambda_{\overline{a b}} .
$$

The expression for $J_{a \bar{b}}$ is exactly as expected. The nonzero expression for $J_{a b}$ is interesting, but not really illuminating. It tells us that in order to make contact with supersymmetric DFT, where $J_{a b}$ is usually taken to vanish, one should make a compensating $\lambda_{a b}$ transformation to cancel the dilatino term. Naturally such a choice of $\lambda$ will in turn affect the gravitino and dilatino transformations as well as the algebra of supersymmetry transformations.

The gravitino transformation is a bit more complicated. A direct computation leads to

$$
\begin{aligned}
& \delta \Psi_{\bar{a}}^{\beta}=\mathcal{D}_{\bar{a}} \epsilon^{\beta}+\Psi_{\bar{a}}^{\gamma} \mathcal{D}_{\gamma} \epsilon^{\beta}+\frac{1}{10} \kappa\left(\epsilon \gamma^{b} \Psi_{\bar{a}}\right)\left(\gamma_{b} \chi\right)^{\beta}-\frac{1}{4} \Psi_{\bar{a}}^{\gamma}\left(\gamma^{c d}\right)_{\gamma}{ }^{\beta} \lambda_{c d}-\lambda_{\bar{a}}{ }^{\bar{b}} \Psi_{\bar{b}}{ }^{\beta}, \\
& =\stackrel{\circ}{\mathcal{D}}_{\bar{a}} \epsilon^{\beta}+\frac{1}{10} \kappa\left(\epsilon \gamma^{b} \Psi_{\bar{a}}\right)\left(\gamma_{b} \chi\right)^{\beta}-\frac{1}{4} \Psi_{\bar{a}}^{\gamma}\left(\gamma^{c d}\right)_{\gamma}{ }^{\beta} \lambda_{c d}-\lambda_{\bar{a}}{ }^{\bar{b}} \Psi_{\bar{b}}{ }^{\beta} \text {. }
\end{aligned}
$$

In going from the first to the second line, we have combined the two derivative terms to give $\stackrel{\circ}{\mathcal{D}}_{\bar{a}}=V_{\bar{a}} \hat{m}^{\hat{m}} \mathcal{D}_{\hat{m}}$ using (5.25). This is purely a bosonic derivative of $\epsilon$, with the $\theta$ derivative cancelling out. This is exactly what needed to happen. Actually, this is a little bit subtle because $\mathcal{D}$ carries the spin connection, which we have not yet defined. We will come back to this in the next section.

For the dilatino, we find

$$
\begin{aligned}
\delta \chi_{a}{ }^{\beta}= & \stackrel{\circ}{\mathcal{D}}_{a} \epsilon^{\beta}+\kappa\left(\epsilon \gamma^{b} \chi_{a}\right) \chi_{b}{ }^{\beta}-\frac{1}{2} \kappa\left(\epsilon \gamma_{a} \chi^{b}\right) \chi_{b}{ }^{\beta}+\frac{1}{2} \kappa\left(\epsilon \gamma_{a} \Psi^{\bar{b}}\right) \Psi_{\bar{b}}{ }^{\beta} \\
& +\lambda_{a}{ }^{b} \chi_{b}{ }^{\beta}-\frac{1}{4} \chi_{a}{ }^{\gamma}\left(\gamma^{c d}\right)_{\gamma}{ }^{\beta} \lambda_{c d}-\lambda_{a}{ }^{\beta}
\end{aligned}
$$

The last term is the compensating tangent space transformation that we will use to maintain the gauge $\chi_{a}{ }^{\alpha}=\frac{1}{10}\left(\gamma_{a}\right)^{\alpha \beta} \chi_{\beta}$. Contracting with a $\gamma$ matrix collapses this expression to

$$
\delta \chi_{\alpha}=\left(\gamma^{a}\right)_{\alpha \beta} \stackrel{\circ}{\mathcal{D}}_{a} \epsilon^{\beta}-\frac{3}{200} \kappa\left(\epsilon \gamma^{a b} \chi\right)\left(\gamma_{a b} \chi\right)_{\alpha}+\frac{1}{20} \kappa(\epsilon \chi) \chi_{\alpha}+\frac{1}{4} \lambda^{a b}\left(\gamma_{a b} \chi\right)_{\alpha}+\frac{1}{2} \kappa\left(\epsilon \gamma^{a} \Psi^{\bar{b}}\right)\left(\gamma_{a} \Psi_{\bar{b}}\right)_{\alpha}
$$

We have not yet addressed how to define the component dilation $\phi$. Recall the superdilaton $\Phi$ transforms as (3.9b). Decomposing indices and neglecting the transport term, this reads

$$
\delta_{\text {g.c. }} \log \Phi=\partial_{\hat{m}} \xi^{\hat{m}}-\partial_{\mu} \xi^{\mu}+\partial^{\mu} \tilde{\xi}_{\mu} \text {. }
$$

At the component level, the last term can be dropped because we take $\partial^{\mu}=0$, but the second term remains problematic as it will obstruct the construction of a sensible supersymmetry transformation. The solution is to shift the definition of the component dilaton relative to the superdilaton:

$$
\phi=\Phi \times \operatorname{det} \phi_{\mu}{ }^{\alpha} \quad \Longrightarrow \quad \delta \log \phi=\partial_{\hat{m}} \xi^{\hat{m}}+2 \partial^{\mu} \tilde{\xi}_{\mu}
$$


Now the second term drops out when $\partial^{\mu}=0$ and the component dilaton transforms as a scalar field. ${ }^{13}$

We can easily work out the supersymmetry transformation of the proposed component dilaton. Observe that

$$
\delta \log \operatorname{det} \phi_{\mu}{ }^{\alpha}=\phi_{\alpha}{ }^{\mu} \delta \phi_{\mu}{ }^{\alpha}=\left(\stackrel{\circ}{\mathcal{J}}_{0}\right)_{\alpha}{ }^{\alpha}=\mathcal{D}_{\alpha} \epsilon^{\alpha}-\kappa \epsilon^{\alpha} \chi_{\alpha} .
$$

Combining with the transformation of the superdilaton,

$$
\delta \log \Phi=\xi^{\mathcal{A}} \mathcal{T}_{\mathcal{A}}+\nabla_{\mathcal{A}} \xi^{\mathcal{A}}(-1)^{a}=-\mathcal{D}_{\alpha} \epsilon^{\alpha}
$$

we recover the expected component transformation,

$$
\delta \log \phi=-\kappa \epsilon^{\alpha} \chi_{\alpha} .
$$

\subsection{The component spin connection}

The expressions for the gravitino and dilatino supersymmetry transformations involve the component spin connection, which we will now specify how to compute. The form of these transformations suggest we should define ${ }^{14}$

$$
\omega_{\hat{a} \hat{b} \hat{c}}:=\Omega_{a \hat{b} \hat{c}}+\Psi_{\hat{a}}{ }^{\alpha} \Omega_{\alpha \hat{b} \hat{c}}=\left(\mathcal{V}_{\Psi}\right)_{\hat{a}}{ }^{\mathcal{D}} \Omega_{\mathcal{D} \hat{b} \hat{c}} .
$$

These are precisely the corrections needed so that under Lorentz transformations, only a bosonic derivative of the Lorentz parameter appears. The key feature of (5.44) is that it allows us to extend the simple relation $\stackrel{\circ}{D}_{\hat{a}}=\left(\mathcal{V}_{\Psi}\right)_{\hat{a}}{ }^{\mathcal{B}} D_{\mathcal{B}}$ to the covariant derivative as well. This is crucial when we consider how to translate the superspace torsion tensor to components.

Let's work this out now. Arbitrary variations $\mathcal{J}$ and $J$ of the supervielbein $\mathcal{V}$ and component vielbein $V$ are given by

$$
\mathcal{J}_{\mathcal{A B}}=\left(\mathcal{V}^{-1} \delta \mathcal{V}\right)_{\mathcal{A}}{ }^{\mathcal{C}} \eta_{\mathcal{C B}}, \quad J_{\hat{a} \hat{b}}=\left(V^{-1} \delta V\right)_{\hat{a}}{ }^{\hat{}} \eta_{\hat{c} \hat{b}}
$$

Using the explicit form of $\mathcal{V}$, these are related by

$$
J_{\hat{a} \hat{b}}=\left(\mathcal{V}_{\Psi}\right)_{\hat{b}}^{\mathcal{B}}\left(\mathcal{V}_{\Psi}\right)_{\hat{a}}^{\mathcal{A}} \mathcal{J}_{\mathcal{A B}}
$$

This is for an arbitrary variation, but applies equally well if we take a derivative. Choosing the variation $\delta$ to be action of the flattened component derivative $\stackrel{\circ}{D}_{\hat{a}}=\left(\mathcal{V}_{\Psi}\right)_{\hat{a}}{ }^{\mathcal{B}} D_{\mathcal{B}}$, we find that

$$
\left(J_{\hat{c}}\right)_{\hat{b} \hat{a}}=\left(\mathcal{V}_{\Psi}\right)_{\hat{a}}^{\mathcal{A}}\left(\mathcal{V}_{\Psi}\right)_{\hat{b}}^{\mathcal{B}}\left(\mathcal{V}_{\Psi}\right)_{\hat{c}}^{\mathcal{C}}\left(\mathcal{J}_{\mathcal{C}}\right)_{\mathcal{B} \mathcal{A}}
$$

\footnotetext{
${ }^{13}$ Another way of arriving at this same conclusion is to recall that the component DFT dilaton is related to the supergravity dilaton by a factor of $e=\operatorname{det} e_{\mathrm{m}}{ }^{\mathrm{a}}$, that is, $\phi=e e^{-2 \varphi}$. The superdilaton in Siegel's superspace DFT is similarly related to the conventional (non-density) superspace dilaton by a factor of $E=\operatorname{sdet} E_{\mathrm{M}}{ }^{\mathrm{A}}$, i.e. $\Phi=E e^{-2 \varphi}$. In conventional superspace, the component and superspace dilatons coincide (hence both are $\varphi$ above). This implies that $\phi=\Phi \times e / E=\Phi \times \operatorname{det} \phi_{\mu}{ }^{\alpha}$.

${ }^{14}$ This definition can equivalently be written as $\omega_{\hat{m}}=\left(\mathcal{V}_{\Xi}^{-1}\right)_{\hat{m}}{ }^{\mathcal{N}} \Omega_{\mathcal{N}}$. It is interesting that this is not the simple $\omega_{m}=\Omega_{m}$ relation from conventional superspace connections, and is another example of how DFT differs.
} 
where $\mathcal{J}_{\mathcal{C}}$ is built from $D_{\mathcal{C}}$. This means that the component flux tensor $F_{\hat{c} \hat{b} \hat{a}}$ is related to the superspace one $\mathcal{F}_{\mathcal{C B A}}$ just by contracting with $\mathcal{V}_{\Psi}$ factors. The same turns out to be true for the component Lorentz connection: here it is crucial that $\left(\mathcal{V}_{\Psi}\right)_{\mathcal{A} \beta}$ vanishes so that only the doubled Lorentz connection from superspace contributes. In other words, the expression (5.47) holds just as well for the covariant derivative. Then the component torsion tensor is related to the superspace one via the extremely simple result

$$
T_{\hat{c} \hat{b} \hat{a}}=\left(\mathcal{V}_{\Psi}\right)_{\hat{a}}^{\mathcal{A}}\left(\mathcal{V}_{\Psi}\right)_{\hat{b}}^{\mathcal{B}}\left(\mathcal{V}_{\Psi}\right)_{\hat{c}}{ }^{\mathcal{C}} \mathcal{T}_{\mathcal{C B} \mathcal{A}}
$$

This expression can be read in one of two ways. The most obvious is as a constraint equation on the component torsion $T_{\hat{c} \hat{b} \hat{a}}$ in terms of the constrained superspace torsion. The other way is to forget briefly that $\mathcal{T}_{\hat{c} \hat{b} \hat{a}}$ is constrained to vanish and instead read this as its definition in terms of the component torsion $T_{\hat{c} \hat{b} \hat{a}}$ and the subleading gravitino corrections involving $\mathcal{T}_{\gamma \hat{b} \hat{a}}, \mathcal{T}_{\gamma \beta \hat{a}}$, and so forth. In this way, the resulting object $\mathcal{T}_{\hat{c} \hat{b} \hat{a}}$ is called the supercovariant torsion tensor; at the component level, it contains the necessary gravitino (and dilatino) additions to $T_{\hat{c} \hat{b} \hat{a}}$ to render it supercovariant, meaning that it transforms under supersymmetry without a derivative of the parameter $\epsilon$. Setting $\mathcal{T}_{\hat{c} \hat{b} \hat{a}}$ to zero is then understood as the supercovariantized version of the bosonic torsion constraint. Since we're mainly interested in figuring out how $\omega$ is constrained here, the first point of view suffices, but the second will be important to keep in mind because we will need to return to the concept of supercovariant curvatures soon.

Expanding out (5.48) using the explicit expression for $\mathcal{V}_{\Psi}$ gives the relation between the component torsion tensor $T_{\hat{c} \hat{a} \hat{b}}$ and its supercovariantized version $\mathcal{T}_{\hat{c} \hat{a} \hat{b}}$. Using the constraints imposed on the superspace torsion, we find

$$
T_{a b c}=\frac{3}{100} \kappa\left(\chi \gamma_{a b c} \chi\right), \quad T_{\bar{a} b c}=\frac{1}{5} \kappa\left(\Psi_{\bar{a}} \gamma_{b c} \chi\right), \quad T_{a \overline{b c}}=-\kappa\left(\Psi_{\bar{b}} \gamma_{a} \Psi_{\bar{c}}\right), \quad T_{\overline{a b c}}=0 .
$$

These give fermion bilinear corrections to the component spin connections. The first and the last constrain only the antisymmetric parts of the respective spin connections; the trace constraints are found in the dilaton torsion, and the hook irrep is undetermined as usual.

Deriving the constraints on the dilaton torsion is a good deal more involved. At the component level, the dilaton torsion is

$$
T_{\hat{a}}=\stackrel{\circ}{D}_{\hat{a}} \log \phi+J_{\hat{b} \hat{a}}^{\hat{b}}
$$

where here the currents are understood to be built using the covariant derivative. Using the definition for the superdilaton torsion tensor (4.9) and the relation (5.40) between the component $\phi$ and the superdilaton $\Phi$, this can be rewritten

$$
T_{a}=\left(\mathcal{V}_{\Psi}\right)_{a}^{\mathcal{A}}\left(\mathcal{T}_{\mathcal{A}}+\mathcal{T}_{\mathcal{A} \beta}{ }^{\beta}+\nabla_{\mathcal{A}} \phi_{\mu}{ }^{\beta} \phi_{\beta}{ }^{\mu}+2 \mathcal{J}^{\beta}{ }_{\beta \mathcal{A}}-\mathcal{J}_{\mathcal{A} \beta}{ }^{\beta}-\mathcal{J}^{\hat{b}}{ }_{\hat{b} \mathcal{A}}\right)+J^{\hat{b}} \hat{b}
$$

A number of simplifications are now needed. First, we will use (5.47) to rewrite $J^{\hat{b}} \hat{a} \hat{a}$ in terms of $\mathcal{J}_{\mathcal{C B} \mathcal{A}}$. Second, we will use $\phi_{\alpha}{ }^{\mu} \delta \phi_{\mu}{ }^{\alpha}=\dot{\mathcal{J}}_{\alpha}{ }^{\alpha}=\mathcal{J}_{\alpha}{ }^{\mathcal{B}}\left(\mathcal{V}_{\Psi}^{-1}\right)_{\mathcal{B}}{ }^{\alpha}$. Finally, we will need to explicitly use the expression (5.26) for $D^{\beta}$ to rewrite $\mathcal{J}_{\beta \mathcal{A}}^{\beta}$ in terms of $\mathcal{J}_{\hat{b} \beta \mathcal{A}}$ and $\mathcal{J}_{\gamma \beta \mathcal{A}}$. (For 
this to work, it's crucial that the $\Omega^{\beta}$ connections end up dropping out of these particular expressions.) Making all these substitutions, one finds the currents nontrivially recombine to form torsion tensors. The end result is

$$
T_{\hat{a}}=\mathcal{T}_{\hat{a}}+\mathcal{T}_{\hat{a} \beta}^{\beta}+\Psi_{\hat{a}}{ }^{\alpha}\left(\mathcal{T}_{\alpha}+\mathcal{T}_{\alpha \beta}{ }^{\beta}\right)+\Psi^{\hat{b} \alpha} \mathcal{T}_{\alpha \hat{b} \hat{a}}-\Psi_{\hat{a}}{ }^{\alpha} \Psi^{\hat{b} \beta} \mathcal{T}_{\beta \alpha \hat{b}}
$$

This provides the dictionary between the component $T_{\hat{a}}$ and the superfield $\mathcal{T}_{\hat{a}}$. In accord with the usual supergravity terminology, we can call the expression for $\mathcal{T}_{\hat{a}}$ the supercovariant dilaton torsion, in comparison with the usual dilaton torsion $T_{\hat{a}}$. Using the explicit expressions for the torsion tensors,

$$
T_{a}=-\kappa \chi_{a}{ }^{\alpha} \chi_{b}{ }^{\beta}\left(\gamma^{b}\right)_{\alpha \beta}=0, \quad T_{\bar{a}}=-\kappa \Psi_{\bar{a}}{ }^{\alpha} \chi_{b}{ }^{\beta}\left(\gamma^{b}\right)_{\alpha \beta}=-\kappa\left(\Psi_{\bar{a}} \chi\right) .
$$

From these expressions, one can derive the trace contributions to $\omega^{b}{ }_{b a}$ and $\omega^{\bar{b}} \overline{b a}$.

\subsection{Supercovariant gravitino curvatures and fermionic equations of motion}

We briefly have mentioned that $\mathcal{T}_{\hat{a}}$ and $\mathcal{T}_{\hat{a} \hat{b} \hat{c}}$ are supercovariant torsion tensors and we found the relations between them and the component analogues. We're going to need to repeat that for the gravitino curvature $\mathcal{T}_{\hat{a} \hat{b}}{ }^{\gamma}$ and the Lorentz curvature $\mathcal{R}_{\hat{a} \hat{b} \hat{c} \hat{d}}$ in order to understand how the superspace geometry reproduces equations of motion in a supercovariant form.

Let's start with the fermionic equations of motion. We will need to build supercovariant gravitino curvatures that generalize $\stackrel{\circ}{D}_{[\bar{c}} \Psi_{\bar{b}]}^{\alpha}$ and $\stackrel{D}{D}^{\bar{b}} \Psi_{\bar{b}}{ }^{\alpha}$. These are going to be related to $\mathcal{T}_{\overline{c b}}{ }^{\alpha}$ and $\mathcal{T}^{\alpha}$. Let's start with the component gravitino (and dilatino) flux. The current $\mathcal{J}$ we want, built with a flat component derivative $\stackrel{\circ}{D}_{\hat{c}}$, is

$$
\left(\stackrel{\circ}{\mathcal{C}}_{\hat{c}}\right)_{\hat{b}}^{\alpha}:=\left(\stackrel{\circ}{D}_{\hat{c}} \mathcal{V}_{\Psi} \mathcal{V}_{\Psi}^{-1}\right)_{\hat{b}}^{\alpha}=\stackrel{\circ}{D}_{\hat{c}} \Psi_{\hat{b}}^{\alpha} \text {. }
$$

Here we leave the $\Omega$ connections out to begin with, as these will turn out to be non-trivial. The above current is also given by

$$
\left(\stackrel{\mathcal{J}}{\hat{c}}_{\hat{b}}{ }^{\alpha}=\left(\mathcal{V}_{\Psi}\right)_{\hat{c}}{ }^{\mathcal{C}} \times\left(\mathcal{V}_{\Psi} \mathcal{J}_{\mathcal{C}} \mathcal{V}_{\Psi}^{-1}\right)_{\hat{b}}{ }^{\alpha}\right.
$$

where $\left(\mathcal{J}_{\mathcal{C}}\right)_{\mathcal{B}}{ }^{\mathcal{A}}$ is a superspace current built by acting with $D_{\hat{C}}$. A straightforward but laborious computation shows that

$$
\begin{aligned}
2 \stackrel{\circ}{[\hat{c}}_{\hat{c} \Psi_{\hat{b}]}{ }^{\alpha}=} & \mathcal{F}_{\hat{c} \hat{b}}{ }^{\alpha}+2 \Psi_{[\hat{c}}{ }^{\gamma} \mathcal{F}_{\gamma \hat{b}]}{ }^{\alpha}-\mathcal{F}_{\hat{c} \hat{b}}{ }^{\hat{a}} \Psi_{\hat{a}}{ }^{\alpha} \\
& -\Psi_{\hat{c}}^{\gamma} \Psi_{\hat{b}}^{\beta} \mathcal{F}_{\beta \gamma}{ }^{\alpha}-2 \Psi_{[\hat{c}}{ }^{\gamma} \mathcal{F}_{\gamma \hat{b}]}{ }^{\hat{a}} \Psi_{\hat{a}}{ }^{\alpha}-\frac{1}{2}\left(\Psi^{T} \Psi\right)^{\alpha \beta} \mathcal{F}_{\beta \hat{c} \hat{b}} \\
& +\Psi_{\hat{c}}^{\gamma} \Psi_{\hat{b}}^{\beta} \mathcal{F}_{\beta \gamma}{ }^{\hat{a}} \Psi_{\hat{a}}{ }^{\alpha}+\left(\Psi^{T} \Psi\right)^{\alpha \beta} \Psi_{\hat{c}}{ }^{\gamma} \mathcal{F}_{\gamma \beta \hat{b}} \\
& +\frac{1}{2}\left(\Psi^{T} \Psi\right)^{\alpha \delta} \Psi_{\hat{c}}{ }^{\gamma} \Psi_{\hat{b}}{ }^{\beta} \mathcal{F}_{\beta \gamma \delta} .
\end{aligned}
$$

Restoring the $\Omega$ connections in the superspace flux tensors on the right-hand side above leads to

$$
\begin{aligned}
2 \stackrel{\circ}{\mathcal{D}}_{[\hat{c}} \Psi_{\hat{b}]}{ }^{\alpha}+2 \omega_{[\hat{c} \hat{b}]}{ }^{\alpha}+\omega^{\alpha}{ }_{\hat{c} \hat{b}}= & \mathcal{T}_{\hat{c} \hat{b}}{ }^{\alpha}+2 \Psi_{[\hat{c}}^{\gamma} \mathcal{T}_{\gamma \hat{b}]}{ }^{\alpha}-\mathcal{T}_{\hat{c} \hat{b}}{ }^{\hat{a}} \Psi_{\hat{a}}{ }^{\alpha} \\
& -\Psi_{\hat{c}}^{\gamma} \Psi_{\hat{b}}^{\beta} \mathcal{T}_{\beta \gamma}{ }^{\alpha}-2 \Psi_{[\hat{c}}{ }^{\gamma} \mathcal{T}_{\gamma \hat{b}]}{ }^{\hat{a}} \Psi_{\hat{a}}{ }^{\alpha}-\frac{1}{2}\left(\Psi^{T} \Psi\right)^{\alpha \beta} \mathcal{T}_{\beta \hat{c} \hat{b}} \\
& +\Psi_{\hat{c}}{ }^{\gamma} \Psi_{\hat{b}}^{\beta} \mathcal{T}_{\beta \gamma}{ }^{\hat{a}} \Psi_{\hat{a}}{ }^{\alpha}+\left(\Psi^{T} \Psi\right)^{\alpha \beta} \Psi_{\hat{c}} \mathcal{T}_{\gamma \beta \hat{b}} \\
& +\frac{1}{2}\left(\Psi^{T} \Psi\right)^{\alpha \delta} \Psi_{\hat{c}}{ }^{\gamma} \Psi_{\hat{b}}{ }^{\beta} \mathcal{T}_{\beta \gamma \delta} .
\end{aligned}
$$


On the left-hand side we have collected the non-Lorentz tangent space component connection $\omega_{\hat{c} \hat{b}}{ }^{\alpha}$ as well as a component of $\omega_{\mathcal{A} \hat{c} \hat{b}}$ in the dual fermionic direction. These are necessary for complete covariance under tangent space transformations. The last term on the left is notable. Inspired by (5.44), we have extended the definition of $\omega_{\hat{a}}$ to $\omega_{\mathcal{A}}$ via

$$
\omega_{\mathcal{A B C}}:=\left(\mathcal{V}_{\Psi}\right)_{\mathcal{A}}{ }^{\mathcal{D}} \Omega_{\mathcal{D} B \mathcal{C}}
$$

The object $\omega^{\alpha} \hat{c} \hat{b}$ appearing above turns out to be gauge covariant under double Lorentz transformations, because its transformation involves only $\partial^{\mu} \lambda_{\hat{c} \hat{b}}$, which vanishes. So the above expression is completely covariant under double Lorentz transformations, although not under the full $\mathcal{H}$ tangent space group, as we have gauge fixed the superspace vielbein.

After imposing subleading torsion constraints, (5.57) collapses to

$$
2 \stackrel{\mathcal{D}}{[\hat{c}}_{\hat{b}]}{ }^{\alpha}+2 \omega_{[\hat{c} \hat{b}]}^{\alpha}+\omega^{\alpha}{ }_{\hat{c} \hat{b}}=\mathcal{T}_{\hat{c} \hat{b}}{ }^{\alpha}+\Psi_{\hat{c}}^{\gamma} \Psi_{\hat{b}}^{\beta} \mathcal{T}_{\beta \gamma}{ }^{\hat{a}} \Psi_{\hat{a}}{ }^{\alpha}+\left(\Psi^{T} \Psi\right)^{\alpha \beta} \Psi_{\hat{c}}^{\gamma} \mathcal{T}_{\gamma \beta \hat{b}}
$$

We can view this as a definition of the supercovariant gravitino curvature $\mathcal{T}_{\hat{c} \hat{b}}{ }^{\alpha}$, which is subsequently set to zero as a superspace constraint. In the component theory, some of this is a conventional constraint, defining what we mean by the fermionic component connections $\omega_{\hat{c} \hat{b}}^{\alpha}$ and $\omega_{\hat{c} \hat{b}}^{\alpha}$, and some of it leads to the fermionic equations of motion. We will see how this works in just a moment.

The above computation can be repeated for $D^{\hat{b}} \Psi_{\hat{b}}{ }^{\alpha}$. The result is

$$
\begin{aligned}
\stackrel{\mathcal{D}}{ }^{\hat{b}} \Psi_{\hat{b}}{ }^{\alpha}+\omega^{\hat{b}}{ }_{\hat{b}}^{\alpha}+\frac{1}{2} \omega^{\beta}{ }_{\hat{b} \hat{c}}\left(\gamma^{\hat{b} \hat{c}}\right)_{\beta}{ }^{\alpha}= & \mathcal{T}^{\alpha}+\mathcal{T}_{\beta}^{\beta}{ }^{\alpha}-\Psi^{\hat{b} \alpha} \mathcal{T}_{\hat{b}}-\Psi^{\hat{b} \alpha} \mathcal{T}_{\gamma \hat{b}}^{\gamma}+\Psi^{\hat{b} \gamma} \mathcal{T}_{\gamma \hat{b}}{ }^{\alpha} \\
& -\frac{1}{2}\left(\Psi^{T} \Psi\right)^{\alpha \beta}\left(\mathcal{T}_{\beta}+\mathcal{T}^{\gamma}{ }_{\gamma \beta}\right)+\Psi^{\hat{b} \alpha} \Psi^{\hat{c} \gamma} \mathcal{T}_{\gamma \hat{b} \hat{c}}+\frac{1}{2}\left(\Psi^{T} \Psi\right)^{\alpha \beta} \Psi^{\hat{b} \gamma} \mathcal{T}_{\gamma \beta \hat{b}} .
\end{aligned}
$$

Imposing the subleading torsion constraints collapses this to

$$
\stackrel{\mathcal{D}}{ }^{\hat{b}} \Psi_{\hat{b}}{ }^{\alpha}+\omega^{\hat{b}} \hat{b}^{\alpha}+\frac{1}{2} \omega^{\beta} \hat{b}_{\hat{c}}\left(\gamma^{\hat{b} \hat{c}}\right)_{\beta}{ }^{\alpha}=\mathcal{T}^{\alpha}+\Psi^{\hat{b} \alpha} \Psi^{\hat{c} \gamma} \mathcal{T}_{\gamma \hat{b} \hat{c}}+\frac{1}{2}\left(\Psi^{T} \Psi\right)^{\alpha \beta} \Psi^{\hat{b} \gamma} \mathcal{T}_{\gamma \beta \hat{b}}
$$

This defines the supercovariant tensor $\mathcal{T}^{\alpha}$.

Let's see how these various tensors decompose. Starting with (5.57), we see that the expression $\mathcal{T}_{\overline{c b}}{ }^{\alpha}$ defines what we might call the supercovariant gravitino curvature. However, this expression is actually constrained to vanish from superspace. This does not mean that the gravitino is pure gauge, of course; it just acts as a definition of $\omega^{\alpha}{ }_{\overline{c b}}$ in terms of $\mathcal{D}_{[\bar{c}} \Psi_{\bar{b}]}{ }^{\alpha}$, which is the closest thing to a gravitino curvature, see (5.65) below. Why is it not an actual curvature? From the transformation law of $\Omega$, one can show that $\delta \omega^{\alpha} \overline{b c}=\Psi^{\bar{a} \alpha} \Lambda_{\bar{a} \mid \overline{b c}}$. From the explicit form of $\omega^{\alpha} \overline{b c}$, this transformation comes from the shift symmetry of the undetermined piece of the spin connection.

Next, let's consider $\mathcal{T}_{\bar{c} b}{ }^{\alpha}$. It is given by ${ }^{15}$

$$
\stackrel{\circ}{\mathcal{D}}_{\bar{c}} \chi_{b}{ }^{\alpha}-\stackrel{\circ}{\mathcal{D}}_{b} \Psi_{\bar{c}}{ }^{\alpha}+\omega_{\bar{c} b}{ }^{\alpha}=\mathcal{T}_{\bar{c} b}{ }^{\alpha}+\Psi_{\bar{c}}^{\gamma} \chi_{b}{ }^{\beta} \mathcal{T}_{\beta \gamma}{ }^{a} \chi_{a}{ }^{\alpha}+\frac{1}{2}\left(\Psi^{T} \Psi\right)^{\alpha \beta} \Psi_{\bar{c}}^{\gamma} \mathcal{T}_{\gamma \beta b}
$$

\footnotetext{
${ }^{15}$ Here and below $\Psi^{T} \Psi$ still involve both $\Psi$ and $\chi$.
} 
Again, $\mathcal{T}_{\bar{c} b}{ }^{\alpha}$ is constrained to vanish in superspace. The part of this that is $\gamma$-traceless in $b \alpha$ just defines $\omega_{\bar{c} b}{ }^{\alpha}$. To find the residual part, we contract with a $\gamma$ matrix, giving

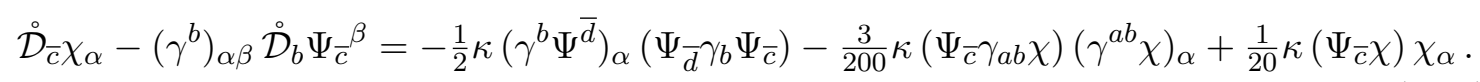

This is the supercovariantized gravitino equation of motion.

Finally we take $\mathcal{T}_{c b}{ }^{\alpha}$ and $\mathcal{T}^{\alpha}$ in tandem. Multiplying $\mathcal{T}_{c b}{ }^{\alpha}$ by $\frac{1}{2} \gamma^{c b}$ gives the same combination of $\omega$ 's as in $\mathcal{T}^{\alpha}$, which lets us remove them. Fixing $\mathcal{T}_{c b}{ }^{\alpha}$ and $\mathcal{T}^{\alpha}$ to zero leads to

$$
\left(\gamma^{b}\right)^{\alpha \beta} \stackrel{\circ}{\mathcal{D}}_{b} \chi_{\beta}-\stackrel{\mathcal{D}}{\bar{b}}^{\bar{b}} \Psi_{\bar{b}}^{\alpha}=-\frac{1}{2} \kappa \Psi^{\bar{b} \alpha}\left(\Psi_{\bar{b}} \chi\right)+\frac{1}{20} \kappa\left(\gamma_{c b} \Psi^{\bar{d}}\right)^{\alpha}\left(\Psi_{\bar{d}} \gamma_{c b} \chi\right)
$$

This is the supercovariantized dilatino equation of motion.

For completeness, we give the fermionic $\omega$ 's that we have uncovered:

$$
\begin{aligned}
2 \omega_{[c b]}^{\alpha}+\omega^{\alpha}{ }_{c b} & =\frac{1}{5}\left(\gamma_{[c}{ }^{\alpha \beta} \dot{\mathcal{D}}_{b]} \chi_{\beta}-\frac{1}{500} \kappa\left(\chi \gamma_{c b a} \chi\right)\left(\gamma^{a} \chi\right)^{\alpha}+\frac{1}{10} \kappa \Psi^{\bar{d} \alpha}\left(\Psi_{\bar{d}} \gamma_{c b} \chi\right),\right. \\
\omega^{\alpha} \overline{c b}_{\bar{c}} & =-2 \mathcal{D}_{[\bar{c}} \Psi_{\bar{b}]}^{\alpha}+\kappa\left(\Psi_{\bar{c}} \gamma^{a} \Psi_{\bar{b}}\right)\left(\gamma_{a} \chi\right)^{\alpha}, \\
\omega_{\bar{c} b}{ }^{\alpha} & =\left.\left[\mathcal{D}_{b} \Psi_{\bar{c}}{ }^{\alpha}-\frac{1}{2} \kappa \Psi^{\bar{d} \alpha}\left(\Psi_{\bar{d}} \gamma_{b} \Psi_{\bar{c}}\right)+\frac{3}{800} \kappa\left(\chi \gamma_{b c d} \chi\right) \Psi_{\bar{c}}{ }^{\delta}\left(\gamma^{c d}\right)_{\delta}{ }^{\alpha}\right]\right|_{\text {proj }}
\end{aligned}
$$

The fact the two $\omega$ 's in the first equation are determined only in this combination is a consequence of the $\Lambda_{c, b}{ }^{\alpha}$ shift symmetry since only this combination is invariant under this shift.

\subsection{Supercovariant Lorentz curvatures and bosonic equations of motion}

Our next task is to compute the supercovariant Lorentz curvatures. Using the dictionary that a component connection $h$ is related to a superspace connection $H$ via $h_{\mathcal{A}^{\underline{b}}}:=\left(\mathcal{V}_{\Psi}\right)_{\mathcal{A}}{ }^{\mathcal{B}} H_{\mathcal{B}}$, one can show that

$$
\begin{aligned}
& \left(\mathcal{V}_{\Psi}\right)_{\hat{b}} \mathcal{B}^{\mathcal{B}}\left(\mathcal{V}_{\Psi}\right)_{\hat{c}}{ }^{\mathcal{C}} \mathcal{R}_{\mathcal{C B}} \underline{\underline{a}}=\left[2 \stackrel{\circ}{D}_{\hat{c}} h_{\hat{b}}^{\underline{a}}+F_{\hat{c} \hat{b}} h_{\hat{d}}^{\underline{a}}-\left(\mathcal{T}_{\hat{c} \hat{b} \alpha}+2 \Psi_{\hat{c}}^{\gamma} \mathcal{T}_{\gamma \hat{b} \alpha}-\Psi_{\hat{c}}^{\gamma} \Psi_{\hat{b}}^{\beta} \mathcal{T}_{\beta \gamma \alpha}\right) h^{\alpha \underline{a}}\right.
\end{aligned}
$$

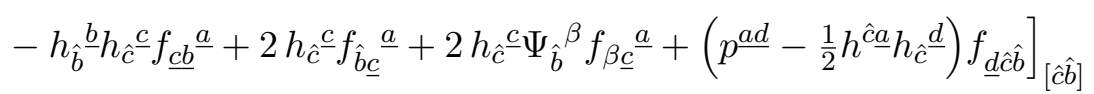

where we have defined the component $p^{\underline{a b}}$ via

$$
p^{\underline{a b}}:=P^{\underline{a b}}+h^{\gamma[\underline{a}} h_{\gamma^{\underline{b}}} .
$$

This expression, like that of $h_{\hat{a}} \underline{\underline{b}}$ can be justified on the grounds that it eliminates fermionic derivatives from the gauge transformation of $p$. The term on the right-hand side of (5.66) that is independent of the fermionic pieces like $\Psi$ and $h^{\alpha}$ we define as $R_{\hat{c} \hat{b}}$, i.e.

$$
\left(\mathcal{V}_{\Psi}\right)_{\hat{b}}{ }^{\mathcal{B}}\left(\mathcal{V}_{\Psi}\right)_{\hat{c}}{ }^{\mathcal{C}} \mathcal{R}_{\mathcal{C B}}{ }^{\underline{a}}=R_{\hat{c} \hat{b}} \underline{\underline{a}}-\left(\mathcal{T}_{\hat{c} \hat{b} \alpha}+2 \Psi_{\hat{c}}^{\gamma} \mathcal{T}_{\gamma \hat{b} \alpha}-\Psi_{\hat{c}}^{\gamma} \Psi_{\hat{b}}^{\beta} \mathcal{T}_{\beta \gamma \alpha}\right) h^{\alpha \underline{a}}+2 h_{\hat{c}} \Psi_{\hat{b}}^{\beta} f_{\beta \underline{c}} \underline{a}^{\underline{a}}
$$

In the above expressions, we have not yet imposed the torsion constraints, so that the reader may check the results in general. 
Now let's specialize to the Lorentz curvature. We won't give all of the full expressions, as only a few are relevant for constructing the equations of motion. First, we observe that the Lorentz curvature with all barred indices requires no fermionic corrections, i.e.

$$
\mathcal{R}_{\overline{d c}} \overline{b a}=R(\omega, h, p) \overline{d c} \overline{b a}
$$

where by $h$ we mean specifically the hook irrep. Recall we constrained the left-hand side of the above to vanish in superspace. At the component level, this works very similarly. The larger representations determine the component $p$ and (parts of) $h$, but these fields drop out of the complete trace. That gives the analogue of the dilaton equation of motion, written purely in terms of the $\mathrm{SO}(9,1)_{R}$ connection:

$$
R(\omega, h, p)_{\overline{b a}}^{\overline{b a}}=R(\omega)_{\overline{b a}}^{\overline{b a}}=0 .
$$

Next we consider a mixed Riemann tensor with one unbarred index:

$$
\mathcal{R}_{d \bar{c} \overline{b a}}=R(\omega, h)_{d \bar{c} \overline{b a}}-\kappa \Psi_{\bar{c}}^{\alpha}\left(\gamma_{d}\right)_{\alpha \beta} \omega^{\beta} \overline{b a} .
$$

Again the constraint from superspace is that this vanishes. Part of this determines $h$. Taking one trace projects out the $h$ terms and gives

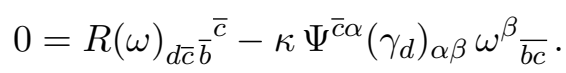

One must plug in the expression for $\omega^{\beta} \overline{b c}$ from (5.65) to give the analogue of the Einstein equation.

While these are perfectly valid equations, they are chiral, being built purely from the right-handed spin connections. To compare e.g. with [13] it would be helpful to have the left-handed versions. These are more involved because the tangent space is quite elaborate in the left-handed sector. To keep things simple, we just look at the two specific representations we want. The first is the complete trace of $\mathcal{R}_{d c b a}$,

$$
\mathcal{R}_{b a}{ }^{b a}=R(\omega)_{b a}^{b a}+\frac{1}{5} \kappa \chi_{\alpha}\left(\gamma^{a b}\right)^{\alpha}{ }_{\beta} \omega^{\beta}{ }_{a b}-\frac{8}{5} \kappa \chi_{\alpha} \omega^{b}{ }_{b}^{\alpha}-2 \kappa \omega_{b}{ }^{\alpha \beta}\left(\gamma^{b}\right)_{\alpha \beta} .
$$

There is a wrinkle here that $\omega_{b}^{\alpha \beta}$ hasn't been determined yet. It arises from analyzing the supercovariant expression for $\mathcal{T}_{\hat{c}}^{\beta \alpha}$. This can be computed from

$$
\begin{aligned}
&\left(\mathcal{V}_{\Psi}\right)_{\hat{c}}{ }^{D} \mathcal{T}_{D}{ }^{\beta \alpha}=\left[\omega_{\hat{c}}^{\beta \alpha}+\mathcal{D}_{\hat{c}} \Psi_{\hat{d}}{ }^{\alpha} \Psi^{\hat{d} \beta}-2 \omega^{\alpha}{ }_{\hat{c}}^{\beta}-2 \Psi^{\hat{d} \alpha} \omega_{\hat{c} \hat{d}}{ }^{\beta}-2 \Psi^{\hat{d} \alpha} \omega^{\beta}{ }_{\hat{c} \hat{d}}\right. \\
&+\frac{1}{4} \Psi_{\hat{c}}{ }^{\gamma} \omega^{\alpha}{ }_{a b}\left(\gamma^{a b}\right)_{\gamma}{ }^{\beta}+\frac{1}{4} \Psi_{\hat{c}}{ }^{\beta} \omega^{\beta}{ }_{a b}\left(\gamma^{a b}\right)_{\gamma}{ }^{\alpha}+2 \Psi^{\hat{d} \alpha} \mathcal{T}_{\hat{c} \hat{d}}{ }^{\beta} \\
&+\left(\Psi^{T} \Psi\right)^{\alpha \gamma} \mathcal{T}_{\hat{c} \gamma}{ }^{\beta}+2 \Psi_{\hat{c}}{ }^{\gamma} \Psi^{\hat{d} \alpha} \mathcal{T}_{\hat{d} \gamma}{ }^{\beta}-\Psi^{\hat{b} \alpha} \Psi^{\hat{d} \beta} \mathcal{T}_{\hat{c} \hat{b} \hat{d}} \\
&+\left(\Psi^{T} \Psi\right)^{\alpha \delta} \Psi_{\hat{c}}{ }^{\gamma} \mathcal{T}_{\gamma \delta}{ }^{\beta}-\left(\Psi^{T} \Psi\right)^{\alpha \delta} \Psi^{\hat{d} \beta} \mathcal{T}_{\delta \hat{c} \hat{d}}-\Psi^{\hat{d} \alpha} \Psi^{\hat{e} \beta} \Psi_{\hat{c}}{ }^{\gamma} \mathcal{T}_{\gamma \hat{d} \hat{e}} \\
&-\left(\Psi^{T} \Psi\right)^{\alpha \delta} \Psi_{\hat{c}}{ }^{\gamma} \Psi^{\hat{d} \beta} \mathcal{T}_{\gamma \delta \hat{d}}-\frac{1}{4}\left(\Psi^{T} \Psi\right)^{\alpha \gamma}\left(\Psi^{T} \Psi\right)^{\beta \delta} \mathcal{T}_{\hat{c} \gamma \delta} \\
&\left.-\frac{1}{4} \Psi_{\hat{c}}{ }^{\gamma}\left(\Psi^{T} \Psi\right)^{\alpha \delta}\left(\Psi^{T} \Psi\right)^{\beta \epsilon} \mathcal{T}_{\gamma \delta \epsilon}\right]^{(\alpha \beta)}
\end{aligned}
$$


This looks fiendishly complicated, but it collapses upon imposing the torsion constraints to

$$
\begin{aligned}
& 0=\mathcal{T}_{\hat{c}}^{\beta \alpha}=\left[\omega_{\hat{c}}^{\beta \alpha}+\mathcal{D}_{\hat{c}} \Psi_{\hat{d}}^{\alpha} \Psi^{\hat{d} \beta}-2 \omega^{\alpha}{ }_{c}^{\beta}-2 \Psi^{d \alpha} \omega_{\hat{c} d}{ }^{\beta}-2 \Psi^{\hat{d} \alpha} \omega^{\beta}{ }_{\hat{c} \hat{d}}\right. \\
&+\frac{1}{4} \Psi_{\hat{c}}{ }^{\gamma} \omega^{\alpha}{ }_{a b}\left(\gamma^{a b}\right)_{\gamma}{ }^{\beta}+\frac{1}{4} \Psi_{\hat{c}}{ }^{\gamma} \omega^{\beta}{ }_{a b}\left(\gamma^{a b}\right)_{\gamma}{ }^{\alpha} \\
&\left.-\kappa\left(\Psi^{T} \Psi\right)^{\alpha \delta} \Psi_{\hat{c}}{ }^{\gamma} \Psi^{d \beta}\left(\gamma_{d}\right)_{\gamma \delta}-\frac{1}{4} \kappa\left(\Psi^{T} \Psi\right)^{\alpha \gamma}\left(\Psi^{T} \Psi\right)^{\beta \delta}\left(\gamma_{c}\right)_{\gamma \delta}\right]^{(\alpha \beta)}
\end{aligned}
$$

This leads to

$$
\begin{aligned}
0= & R(\omega)_{b a}^{b a}-\frac{12}{5} \kappa\left(\chi_{\alpha} \omega^{b}{ }_{b}{ }^{\alpha}-\frac{1}{2}\left(\chi \gamma^{a b}\right)_{\alpha} \omega^{\alpha}{ }_{a b}\right)+\frac{4}{25} \kappa\left(\chi \gamma^{c} \mathcal{D}_{c} \chi\right)+2 \kappa\left(\Psi_{\bar{d}} \gamma^{c} \mathcal{D}_{c} \Psi_{\bar{d}}\right) \\
& +\frac{1}{50} \kappa^{2}\left(\Psi^{\bar{a}} \gamma^{c} \gamma^{d} \chi\right)\left(\Psi_{\bar{a}} \gamma_{d} \gamma_{c} \chi\right)-\frac{1}{100} \kappa^{2}\left(\Psi^{\bar{a}} \gamma^{c} \gamma^{d} \chi\right)\left(\Psi_{\bar{a}} \gamma_{c} \gamma_{d} \chi\right)+\frac{1}{2} \kappa^{2}\left(\Psi^{\bar{a}} \gamma^{c} \Psi^{\bar{b}}\right)\left(\Psi_{\bar{a}} \gamma_{c} \Psi_{\bar{b}}\right)
\end{aligned}
$$

and it helps to use

$$
\omega^{b}{ }_{b}^{\alpha}-\frac{1}{2}\left(\gamma^{a b}\right)^{\alpha}{ }_{\beta} \omega^{\beta}{ }_{a b}=\frac{9}{10}\left(\gamma^{b} \mathcal{D}_{b} \chi\right)^{\alpha}-\frac{1}{20} \kappa\left(\gamma^{c b} \Psi^{\bar{a}}\right)^{\alpha}\left(\Psi_{\bar{a}} \gamma_{c b} \chi\right),
$$

which follows from (5.65) to give

$$
0=R(\omega)_{b a}^{b a}-2 \kappa\left(\chi \gamma^{c} \mathcal{D}_{c} \chi\right)+2 \kappa\left(\Psi_{\bar{d}} \gamma^{c} \mathcal{D}_{c} \Psi_{\bar{d}}\right)+\frac{16}{25}\left(\Psi^{\bar{a}} \chi\right)\left(\Psi_{\bar{a}} \chi\right)+\frac{1}{2}\left(\Psi^{\bar{a}} \Psi^{\bar{b}}\right)\left(\Psi_{\bar{a}} \Psi_{\bar{b}}\right)
$$

Combining this with $R(\omega)_{\overline{b a}} \overline{b a}=0$ gives

$0=R(\omega)_{b a}{ }^{b a}-R(\omega)_{\overline{b a}}{ }^{\overline{b a}}-2 \kappa\left(\chi \gamma^{c} \mathcal{D}_{c} \chi\right)+2 \kappa\left(\Psi^{\bar{d}} \gamma^{c} \mathcal{D}_{c} \Psi_{\bar{d}}\right)+\frac{16}{25}\left(\Psi^{\bar{a}} \chi\right)\left(\Psi_{\bar{a}} \chi\right)+\frac{1}{2}\left(\Psi^{\bar{a}} \Psi^{\bar{b}}\right)\left(\Psi_{\bar{a}} \Psi_{\bar{b}}\right)$.

It is tempting to call this the dilaton field equation, but this is not guaranteed. Superspace identifies only the on-shell locus, so this is actually a combination of the dilaton field equation and the fermionic field equations.

Repeating for the generalized Ricci tensor and skipping the intermediate steps, we find

$$
\begin{aligned}
0= & -\frac{1}{2} R(\omega)_{a}{ }^{\bar{c}} \overline{b c}+\frac{1}{2} R(\omega)_{\bar{b}}{ }^{c}{ }_{a c}-\frac{1}{2}\left(\chi \mathcal{D}_{a} \Psi_{\bar{b}}\right)+\frac{3}{2}\left(\Psi^{\bar{c}} \gamma_{a} \mathcal{D}_{\bar{c}} \Psi_{\bar{b}}\right)-\left(\Psi^{\bar{c}} \gamma_{a} \mathcal{D}_{\bar{b}} \Psi_{\bar{c}}\right)-\frac{2}{5}\left(\Psi_{\bar{b}} \gamma_{a b} \mathcal{D}^{b} \chi\right) \\
& +\frac{1}{10}\left(\chi \gamma_{a b} \mathcal{D}^{b} \Psi_{\bar{b}}\right)+\frac{7}{320}\left(\Psi_{\bar{b}} \gamma^{b c} \chi\right)\left(\Psi^{\bar{d}} \gamma_{a b c} \Psi_{\bar{d}}\right)-\frac{1}{960}\left(\Psi_{\bar{b}} \gamma_{a b c d} \chi\right)\left(\Psi^{\bar{d}} \gamma^{b c d} \Psi_{\bar{d}}\right) .
\end{aligned}
$$

Again, this is only the double vielbein field equation modulo the fermionic field equations.

\subsection{Comparing with component DFT and $\mathcal{N}=1$ supergravity}

Our results for the field equations and supersymmetry transformations look rather different from those found by Jeon, Lee, and Park [13], and it is a crucial check that they actually agree. For the field equations, this is straightforward to verify after making numerous convention swaps. Most of these are obvious, but a few we will highlight. For the fermions, the charge conjugation tensor in [13] is imaginary, leading to

$$
\psi_{\bar{p}} \rightarrow \Psi_{\bar{a}}^{\alpha}, \quad \bar{\psi}_{\bar{p}} \rightarrow i \Psi_{\bar{a}}^{\alpha} .
$$


There is also an additional sign in the definition of the dilatino, so (remembering the charge conjugation has an opposite sign for the opposite chirality)

$$
\rho \rightarrow-\chi_{\alpha}, \quad \bar{\rho} \rightarrow i \chi_{\alpha} .
$$

The $\mathrm{SO}(9,1)_{R}$ metric has the opposite sign, $\eta_{\bar{p} \bar{q}} \rightarrow-\eta_{\overline{a b}}$, and the spin connection uniformly differs by a sign when the indices are lowered

$$
\Phi_{A p q} \rightarrow-\omega_{\hat{m} a b}, \quad \Phi_{A \bar{p} \bar{q}} \rightarrow-\omega_{\hat{m} \overline{a b}} .
$$

We also must fix $\kappa=1$. With these modifications, the fermionic field equations (5.63) and (5.6) coincide exactly with those given in [13], while the bosonic field equations (5.79) and (5.80) hold modulo the former. This is an intricate comparison involving numerous Fierz identities, and we were saved countless hours of computation by using Cadabra [34, 35]. Similarly, the supersymmetry transformations discussed in section 5.4 match, provided we choose the compensating Lorentz parameters in (5.35) so that $J_{a b}=J_{\overline{a b}}=0$.

We may also compare with $10 \mathrm{D} \mathcal{N}=1$ supergravity in the string frame, see e.g. [36]. To keep things simple, we'll just focus on the supersymmetry transformations. Taking the explicit parametrization (2.12) for the double vielbein, we find that

$$
e_{\mathrm{a}}{ }^{\mathrm{m}} \delta e_{\mathrm{mb}}=\frac{1}{10} \kappa\left(\epsilon \gamma_{a b} \chi\right)-\frac{1}{2}\left(\lambda_{a b}-\lambda_{\overline{a b}}\right)-\kappa\left(\epsilon \gamma_{(a} \Psi_{\bar{b})}\right) .
$$

To identify the conventional supersymmetry transformation requires that we choose

$$
\lambda_{a b}=\frac{1}{5} \kappa\left(\epsilon \gamma_{a b} \chi\right)-2 \kappa\left(\epsilon \gamma_{[a} \Psi_{\bar{b}]}\right), \quad \lambda_{\overline{a b}}=0 .
$$

Moreover, one must keep in mind the factor of $1 / \sqrt{2}$ in the covariant derivative, which requires that we rescale the component gravitino, $\psi_{\mathrm{m}}{ }^{\alpha}=\sqrt{2} e_{\mathrm{m}}{ }^{\mathrm{a}} \Psi_{\bar{a}}{ }^{\alpha}$ to have a canonical supersymmetry transformation. All told, this leads to

$$
\delta e_{\mathrm{m}}^{\mathrm{a}}=-\frac{1}{\sqrt{2}} \kappa\left(\epsilon \gamma^{\mathrm{a}} \psi_{\mathrm{m}}\right) .
$$

Matching conventions requires $\kappa=-1 / \sqrt{2}$, which we fix for the remainder of this discussion. The supergravity dilaton $\varphi$ is related to $\phi=e^{-2 d}$ by $\phi=e e^{-2 \varphi}$. This allows us to identify the supergravity dilatino via

$$
\delta \varphi=-\frac{1}{\sqrt{2}} \epsilon \lambda, \quad \lambda_{\alpha}=\frac{1}{2} \chi_{\alpha}-\frac{1}{2 \sqrt{2}}\left(\gamma^{\mathrm{a}} \psi_{\mathrm{a}}\right)_{\alpha} .
$$

We normalize the dilatino $\lambda$ the same way as [36], but we keep the standard $\varphi$, which differs from the dilaton used in [36].

Now let's analyze the fermion variations. The gravitino transformation involves the connection $\omega_{\bar{a} b c}$. This is found from the torsion component

$$
T_{\bar{c} a b}=\frac{1}{2 \sqrt{2}} H_{\mathrm{abc}}-\frac{1}{2 \sqrt{2}}\left(C_{\mathrm{cba}}+C_{\mathrm{abc}}+C_{\mathrm{acb}}\right)+\omega_{\bar{c} a b}
$$


Solving for $\omega$ and translating to $\hat{\omega}$ gives

$$
\omega_{\bar{c} a b}=\frac{1}{\sqrt{2}} \hat{\omega}_{\mathrm{cab}}-\frac{1}{2 \sqrt{2}} H_{\mathrm{abc}}+T_{\bar{c} a b}+\frac{1}{4 \sqrt{2}}\left(\psi_{\mathrm{c}} \gamma_{\mathrm{a}} \psi_{\mathrm{b}}-\psi_{\mathrm{c}} \gamma_{\mathrm{b}} \psi_{\mathrm{a}}+\psi_{\mathrm{a}} \gamma_{\mathrm{c}} \psi_{\mathrm{b}}\right)
$$

where we have used the standard supercovariant torsion tensor from supergravity,

$$
\hat{\omega}_{\mathrm{cba}}=-\frac{1}{2}\left(C_{\mathrm{cba}}+C_{\mathrm{abc}}+C_{\mathrm{acb}}\right)+\frac{1}{4}\left(\psi_{\mathrm{c}} \gamma_{\mathrm{a}} \psi_{\mathrm{b}}\right)-\frac{1}{4}\left(\psi_{\mathrm{c}} \gamma_{\mathrm{b}} \psi_{\mathrm{a}}\right)+\frac{1}{4}\left(\psi_{\mathrm{a}} \gamma_{\mathrm{c}} \psi_{\mathrm{b}}\right)
$$

The field strength $H$ should be supercovariantized to $\hat{H}_{\mathrm{abc}}=H_{\mathrm{abc}}+\frac{3}{2}\left(\psi_{[\mathrm{a}} \gamma_{\mathrm{b}} \psi_{\mathrm{c}]}\right)$ which gives

$$
\omega_{\bar{c} a b}=T_{\bar{c} a b}+\frac{1}{\sqrt{2}}\left(\hat{\omega}_{\mathrm{cab}}-\frac{1}{2} \hat{H}_{\mathrm{cab}}+\psi_{\mathrm{c}} \gamma_{[\mathrm{a}} \psi_{\mathrm{b}]}\right)=T_{\bar{c} a b}+\frac{1}{\sqrt{2}}\left(\hat{\omega}_{\mathrm{cab}}^{-}+\left(\psi_{\mathrm{c}} \gamma_{[\mathrm{a}} \psi_{\mathrm{b}]}\right)\right)
$$

where we have defined $\hat{\omega}^{-}$by the above. The component gravitino transformation then becomes

$$
\delta \psi_{\mathrm{m}}{ }^{\alpha}=\partial_{\mathrm{m}} \epsilon^{\alpha}-\frac{1}{4} \hat{\omega}_{\mathrm{mab}}^{-}\left(\gamma^{\mathrm{ab}} \epsilon\right)^{\alpha}+\frac{1}{\sqrt{2}}\left(\epsilon^{\alpha}\left(\psi_{\mathrm{m}} \lambda\right)-\psi_{\mathrm{m}}{ }^{\alpha}(\epsilon \lambda)+\left(\gamma^{\mathrm{a}} \lambda\right)^{\alpha}\left(\psi_{\mathrm{m}} \gamma_{\mathrm{a}} \epsilon\right)\right) .
$$

This matches [36].

To identify the dilatino transformation, we need

$$
\begin{aligned}
& T_{a b c}=\frac{1}{2 \sqrt{2}}\left(H_{\mathrm{abc}}+3 C_{[\mathrm{abc}]}\right)+3 \omega_{[a b c]} \Longrightarrow \omega_{[a b c]}=\frac{1}{3} T_{[a b c]}+\frac{1}{\sqrt{2}}\left(\hat{\omega}_{[\mathrm{abc}]}^{-}+\frac{1}{3} \hat{H}_{\mathrm{abc}}+\frac{1}{2}\left(\psi_{[\mathrm{a}} \gamma_{\mathrm{b}} \psi_{\mathrm{c}]}\right)\right), \\
& T_{a}=\frac{1}{\sqrt{2}}\left(D_{\mathrm{a}} \log \phi+C_{\mathrm{ab}}^{\mathrm{b}}\right)+\omega^{b}{ }_{b a} \quad \Longrightarrow \quad \omega_{b a}^{b}=T_{a}+\frac{1}{\sqrt{2}}\left(\hat{\omega}^{\mathrm{b}}{ }_{\mathrm{ba}}-D_{\mathrm{a}} \log \phi-\frac{1}{2}\left(\psi_{\mathrm{a}} \gamma^{\mathrm{b}} \psi_{\mathrm{b}}\right)\right) .
\end{aligned}
$$

Putting this together, one finds

$$
\delta \lambda_{\alpha}=-\frac{\sqrt{2}}{4}\left(\gamma^{\mathrm{m}} \epsilon\right)_{\alpha}\left(\partial_{\mathrm{m}} \varphi-\frac{1}{4}\left(\psi_{\mathrm{m}} \lambda\right)\right)-\frac{\sqrt{2}}{48}\left(\gamma^{\mathrm{abc}} \epsilon\right)_{\alpha}\left(\hat{H}_{\mathrm{abc}}-\frac{1}{4}\left(\lambda \gamma_{\mathrm{abc}} \lambda\right)\right)
$$

This also matches [36], keeping in mind that $\left(\bar{\lambda} \gamma_{a b c} \lambda\right)=-\left(\lambda \gamma_{a b c} \lambda\right)$ using our convention for the charge conjugation matrix.

\section{Open questions}

Our initial goal in this work was to reproduce the component results of $\mathcal{N}=1$ DFT using a manifestly supersymmetric starting point. While we have achieved that, at the same time we have stumbled upon an intriguing expansion of the tangent space group. This enlarged tangent space seems necessary in order to explicitly gauge away unphysical components of the supervielbein and suggests the introduction of an ever higher set of connections. These in turn require curvature constraints in order to render them composite, with the consequence that only physical components of curvature tensors appear. As we have emphasized in section 2 , this is not dependent upon supersymmetry per se, but can be imposed even in the bosonic theory, where it is required if we demand that the undetermined part of the spin connection be gauged. 
Several issues remain to be addressed, and we pose these here as questions:

\section{- Is the enlarged tangent space description correct?}

We have proposed a simple Lie superalgebra as the basis for the tangent space group $\left.\widehat{\mathcal{H}}_{L} \times \mathrm{SO(9,1}\right)_{R}$. Here $\widehat{\mathcal{H}}_{L}$ is understood as the dual of the super-Maxwell $\infty$ algebra, and $\widehat{\mathrm{SO}(9,1)_{R}}$ is the dual of the on-shell Maxwell $\infty$ algebra. For the lowest lying levels, this can explicitly be verified, providing the connections necessary for building torsions and curvatures along with the required gauge transformations. What remains to be confirmed is that the higher connections $H_{\mathcal{A}} \underline{\underline{b}}$ and the PoláčekSiegel fields $P^{\underline{a b}}$ are completely determined (modulo gauge symmetries) by imposing constraints on the curvatures. We have checked this explicitly through dimension two, which is sufficient to understand the two derivative equations of motion and supersymmetry transformations. Possibly the proposal we make requires modifications beyond dimension two, or perhaps it is indeed complete to all orders. This will require further investigation.

\section{- Does the $\mathcal{N}=2$ formulation work in a similar way?}

An extension to $\mathcal{N}=2$ double field theory should be possible, in particular to reproduce the component results of $\mathcal{N}=2$ supersymmetric DFT [15]. Superspaces relevant for these cases have been considered by Cederwall [20] and by Hatsuda, Kamimura, and Siegel $[18,19]$. Cederwall's proposal is the more conventional of the two, relying upon an $\operatorname{OSp}(D, D \mid 2 s)$ vielbein and a separate $\Omega$ connection valued just in $\operatorname{Spin}(D-1,1)_{L} \times \operatorname{Spin}(D-1,1)_{R}$. The formulation of Hatsuda et al. employed an enlarged Poláček-Siegel megavielbein directly, but similarly restricted the local tangent space. (In both approaches, there is an elegant interpretation of the RR field strengths lying in the supervielbein.) Both differ from the $\mathcal{N}=1$ approach we have advocated (and its natural $\mathcal{N}=2$ extension), which requires an enhanced tangent space beyond $\operatorname{Spin}(D-1,1)_{L} \times \operatorname{Spin}(D-1,1)_{R}$ to explicitly eliminate the unphysical components of the supervielbein.

Presumably the relationship between our approach (which builds on Siegel's 1993 formulation [2]) and these ones involves a process of "degauging" where one fixes the additional gauge symmetries and reinterprets the gauge-fixed higher connections as curvatures involving undetermined components of the spin connection. This would be very similar to the relationship between conformal gravity (where additional dilation and special conformal connections are introduced) and conventional Poincaré gravity. ${ }^{16}$ In the latter case, when describing conformal gravity, one must require by hand that the local Weyl transformation $\delta e_{\mathrm{m}}{ }^{\mathrm{a}}=-\sigma e_{\mathrm{m}}{ }^{\mathrm{a}}$ be a symmetry of the action. Only the traceless projection of the Riemann tensor — the Weyl tensor — is physical in this scheme, and other pieces are unphysical.

- Can the $\mathcal{N}=2$ formulation tell us about super- $E_{11}$ ?

A major motivation to study the $\mathcal{N}=2$ formulation is to learn something about super- $E_{11}$, whose component formulation has recently been explored [26]. One might

\footnotetext{
${ }^{16}$ See e.g. [37] for a pedagogical discussion of conformal (super)gravity, and [38] for its superspace analogue.
} 
expect that in super-DFT, the RR forms should appear only via their field strengths, but in extending the formalism to include their $p$-form potentials, the link to super$E_{11}$ would be uncovered. Some work along these lines has already been explored by Cederwall, who proposed to identify RR potentials and field strengths using the language of OSp spinors [20]. It would be interesting to explore this further.

\section{- Can higher derivative or off-shell formulations be encoded in super-DFT?}

The formulation of superspace DFT we have discussed is on-shell, much like the conventional 10D $\mathcal{N}=1$ superspace it reduces to when the section condition is imposed. Naturally, this is because it is describing at the component level the supersymmetric DFT of $[12,13]$, which is a two-derivative theory whose supersymmetry transformations close only up to the equations of motion. Two natural questions suggest themselves. First, can we extend superspace DFT to encode higher derivative $\alpha^{\prime}$ corrections? This certainly seems plausible, and relatively recently, the first-order corrections to $\mathcal{N}=1$ supersymmetric DFT were constructed at the component level [39]. It would be quite interesting to understand how this construction can be geometrized in superspace, and if that can aid in its extension to higher orders. An even more ambitious proposal would be to try to understand whether any connection can be drawn to the off-shell pure spinor superspace [28-30], which might allow the direct construction of higher order invariants.

\section{Acknowledgments}

It is a pleasure to thank Falk Hassler, Gianluca Inverso, William Linch, Jakob Palmkvist, Axel Kleinschmidt, and Warren Siegel for discussions and comments. This work is partially supported by the NSF under grants NSF-1820921 and PHY-1606531, and the Mitchell Institute for Fundamental Physics and Astronomy at Texas A\&M University.

\section{A Notations and conventions}

\section{A.1 $\operatorname{Spin}(9,1)$ conventions}

Our conventions for $\operatorname{Spin}(9,1)$ are as follows. The metric $\eta_{a b}$ is mostly positive signature, with $\gamma$-matrices

$$
\left\{\gamma^{a}, \gamma^{b}\right\}=2 \eta^{a b}, \quad \gamma^{a_{1} \cdots a_{n}}:=\gamma^{\left[a_{1}\right.} \cdots \gamma^{\left.a_{n}\right]}
$$

(Anti)-symmetrization always involves factors of $1 / n$ !. We use a Weyl basis for the $\gamma$-matrices so that

$$
\gamma^{a}=\left(\begin{array}{cc}
0 & \left(\gamma^{a}\right)^{\alpha \beta} \\
\left(\gamma^{a}\right)_{\alpha \beta} & 0
\end{array}\right), \quad \gamma_{11}=\left(\begin{array}{cc}
1 & 0 \\
0 & -1
\end{array}\right), \quad C=\left(\begin{array}{cc}
0 & 1 \\
-1 & 0
\end{array}\right)
$$

The chiral (Pauli) $\gamma$-matrices $\left(\gamma^{a}\right)_{\alpha \beta}$ obey the 10D identities

$$
\left(\gamma^{a}\right)_{(\alpha \beta}\left(\gamma_{a}\right)_{\gamma) \delta}=0, \quad\left(\gamma^{a b c}\right)_{[\alpha \beta}\left(\gamma_{a b}\right)_{\gamma]}^{\delta}=0
$$


The gravitino and dilatino decompose as

$$
\psi=\left(\begin{array}{c}
\psi^{\alpha} \\
0
\end{array}\right), \quad \chi=\left(\begin{array}{c}
0 \\
\chi_{\alpha}
\end{array}\right)
$$

We use explicit Weyl-component notation throughout and suppress indices in obvious ways so that, for example,

$$
(\psi \chi)=\psi^{\alpha} \chi_{\alpha}=-(\chi \psi), \quad\left(\psi \gamma_{a b c} \psi\right)=\psi^{\alpha}\left(\gamma_{a b c}\right)_{\alpha \beta} \psi^{\beta}, \quad\left(\chi \gamma_{a b c} \chi\right)=\chi_{\alpha}\left(\gamma_{a b c}\right)^{\alpha \beta} \chi_{\beta},
$$

This last point is important because in Dirac notation with Majorana fermions, we would take $\bar{\Psi}=\Psi^{T} C$, so $\bar{\chi}=\left(-\chi_{\alpha}, 0\right)$ involves a minus sign while $\bar{\psi}=\left(0, \psi^{\alpha}\right)$ does not.

\section{A.2 $\operatorname{OSp}(p, q \mid 2 s)$ conventions}

The supergroup $\operatorname{OSp}(p, q \mid 2 s)$ is the group of linear transformations that preserve the canonical invariant $\eta_{A B}$, graded with $p+q$ bosonic and $2 s$ fermionic indices,

$$
\eta_{A B}=\left(\begin{array}{cc}
\eta_{\hat{a} \hat{b}} & 0 \\
0 & \varepsilon_{\hat{\alpha} \hat{\beta}}
\end{array}\right)
$$

where $\eta_{\hat{a} \hat{b}}$ is the $\operatorname{SO}(p, q)$ metric and $\varepsilon_{\hat{\alpha} \hat{\beta}}$ is the canonical symplectic element $\left(\begin{array}{cc}0 & 1 \\ -1 & 0\end{array}\right)$. We will be interested in the two cases $\operatorname{OSp}(10,10 \mid 32)$ and $\operatorname{OSp}(9,1 \mid 32)$ where $\hat{\alpha}$ is a 32-component Dirac index. The generators $M_{A B}$ of this algebra obey

$$
\left[M_{A B}, M_{C D}\right]=(-)^{a(b+c)} \eta_{B C} M_{A D}-(-)^{b c} \eta_{A C} M_{B D}-(-)^{b c} M_{A C} \eta_{B D}+(-)^{d(b+c)} \eta_{A D} M_{B C}
$$

On the fundamental vector representation $V^{A}$,

$$
\delta_{\lambda} V^{A} \equiv \frac{1}{2} \lambda^{B C} M_{C B} V^{A}=\lambda^{A B} V_{B} \quad \Longrightarrow \quad M_{C B} V^{A}=2 V_{[C} \delta_{B]}^{A}
$$

where we use a NW-SE convention for contracting super-indices, so that $V_{A}=V^{B} \eta_{B A}$.

\section{B Poláček-Siegel formulation of DFT with connections}

In this appendix, we review the Poláček-Siegel approach to connections in DFT [31]. We will take a somewhat more streamlined approach, eliminating the discussion of background derivatives $D_{M}$, while simultaneously extending the allowed gauging beyond the Lorentz group to a generic gauge group. We restrict purely to the field theoretic description of their results and avoid discussing the motivations from the form of current algebras on the string worldsheet.

To simplify notation somewhat, we will denote the doubled coordinates of DFT by $x^{m}$, without any further adornment, and the corresponding tangent space indices by $a, b, \cdots$. 
Now let the coordinates be enhanced to $z^{M}=\left(y^{\underline{m}}, x^{m}, \tilde{y}_{\underline{m}}\right)$ subject to a section condition $\partial^{M} \otimes \partial_{M}=0$ where $\eta^{M N}$ is given by

$$
\eta^{M N}=\left(\begin{array}{ccc}
0 & 0 & \delta \underline{\underline{m}}^{n} \\
0 & \eta^{m n} & 0 \\
\delta_{\underline{m} \underline{\underline{n}}}^{\underline{n}} & 0 & 0
\end{array}\right) .
$$

We will assume the section condition for the additional coordinates is already solved by $\partial^{\underline{m}}=0$, so that no fields depend on $\tilde{y}_{\underline{m}}$. The dependence on $y^{\underline{m}}$ will be chosen in a particular way to parametrize how fields transform under double Lorentz transformations as well as any other local gauge symmetries present. We refer to this local gauge group that extends the double Lorentz group as $\widehat{\mathcal{H}}$. We assume that all coordinates are bosonic to avoid introducing gradings, but it is trivial to extend to supercoordinates.

Let the megavielbein on this space be denoted $\mathcal{V}_{M}{ }^{A}$. It transforms under diffeomorphisms in the usual manner as

$$
\delta \mathcal{V}_{M}^{A}=\xi^{N} \partial_{N} \mathcal{V}_{M}^{A}+\left(\partial_{M} \xi^{N}-\partial^{N} \xi_{M}\right) \mathcal{V}_{M}^{A}
$$

There are no tangent space transformations, as these will be encoded in $\xi$ itself. Rewriting the above transformation in terms of $\xi^{A}=\xi^{M} \mathcal{V}_{M}{ }^{A}$ leads to the covariant form of generalized diffeomorphisms,

$$
\delta \mathcal{V}_{M}{ }^{A}=\mathcal{V}_{M}{ }^{B}\left(\nabla_{B} \xi^{A}-\nabla^{A} \xi_{B}+\xi^{C} \mathcal{T}_{C B}{ }^{A}\right)
$$

where $\nabla_{A}:=\mathcal{V}_{A}{ }^{M} \partial_{M}$. The totally antisymmetric torsion tensor $\mathcal{T}_{C B A}$ is given by

$$
\mathcal{T}_{C B A}=-3 \nabla_{[C} \mathcal{V}_{B}{ }^{M} \mathcal{V}_{M A]}
$$

These obey Bianchi identities

$$
4 \nabla_{[A} \mathcal{T}_{B C D]}+3 \mathcal{T}_{[A B}{ }^{F} \mathcal{T}_{C D] F}=0
$$

Of course, the torsion tensor also appears in the algebra of covariant derivatives

$$
\left[\nabla_{A}, \nabla_{B}\right]=-\mathcal{T}_{A B}^{C} \nabla_{C}
$$

where we have made use of the section condition. The standard form of the Bianchi identity follows from the above,

$$
\left(\nabla_{[A} \mathcal{T}_{B C]}^{D}+\mathcal{T}_{[A B}{ }^{F} \mathcal{T}_{F \mid C]}{ }^{D}\right) \nabla_{D}=0
$$

and coincides with (B.5) upon using the section condition.

Now let us decompose our derivatives as $\nabla_{A}=\left(\nabla_{\underline{a}}, \nabla_{a}, \nabla^{\underline{a}}\right)$. The derivative $\nabla_{\underline{a}}$ will be identified soon with the generator of local $\widehat{\mathcal{H}}$ transformations, and $\nabla_{a}$ will coincide with the usual covariant derivative. The additional derivative $\nabla^{\underline{a}}$ will turn out to be a composite 
operator, which we will explore in due course. We will be interested in the following algebra, where some of the torsion tensors $\mathcal{T}_{C B A}$ have been set to constants $f_{C B A}{ }^{17}$

$$
\begin{aligned}
& {\left[\nabla_{a}, \nabla_{b}\right]=-\mathcal{T}_{a b}{ }^{\underline{c}} \nabla_{\underline{c}}-\mathcal{T}_{a b}{ }^{c} \nabla_{c}-f_{a b \underline{c}} \nabla^{\underline{c}},} \\
& {\left[\nabla_{\underline{a}}, \nabla_{b}\right]=-f_{\underline{a} b}{ }^{c} \nabla_{c}-f_{\underline{a} b}{ }^{\underline{c}} \nabla_{\underline{c}},} \\
& {\left[\nabla_{\underline{a}}, \nabla_{\underline{b}}\right]=-f_{\underline{a b}} \underline{\underline{c}} \nabla_{\underline{c}},} \\
& {\left[\nabla^{\underline{a}}, \nabla^{\underline{b}}\right]=-\mathcal{T}_{c} \underline{a \underline{b}} \nabla^{c}-\mathcal{T}^{a a^{c}} \nabla_{\underline{c}}-f_{\underline{\underline{c}}}^{\underline{a b}} \nabla^{\underline{c}},} \\
& {\left[\nabla^{\underline{a}}, \nabla_{b}\right]=-\mathcal{T}_{b c}{ }^{\underline{a}} \nabla^{c}-f_{b \underline{c}}{ }^{\underline{a}} \nabla^{\underline{c}}+\mathcal{T}_{b}{ }^{\underline{a}} \nabla_{\underline{c}},} \\
& {\left[\nabla^{\underline{a}}, \nabla_{\underline{b}}\right]=-f_{\underline{b c}} \underline{\underline{a}} \nabla^{\underline{c}}-f_{\underline{b}}{ }^{\underline{a}} \nabla^{c}+f_{\underline{\underline{b}}} \underline{\underline{a c}} \nabla_{\underline{c}} .}
\end{aligned}
$$

In addition to setting some of the torsion components to constants, we have also chosen to fix $\mathcal{T}_{\underline{c b a}}$ and $\mathcal{T}_{\underline{c} \underline{a} a}$ to zero. The reason for this choice is that we want $\nabla_{\underline{a}}$ to furnish a closed subalgebra: this is the $\widehat{\mathcal{H}}$ algebra that will be gauged. The torsion tensors that have been set to constants will turn out to correspond to choices of how various components of the megavielbein transform under local $\widehat{\mathcal{H}}$ transformations. The remaining non-constant tensors $\mathcal{T}_{a b c}$ and $\mathcal{T}_{a b}$ 毫 will correspond to the standard torsion tensor and $\widehat{\mathcal{H}}$-curvature, while $\mathcal{T}_{c} \underline{a b}$ and $\mathcal{T}^{a b c}$ will be new tensors, whose role will be explained in due course.

We are not going to explicitly solve the above constraints, but give a megavielbein that does the job. Following Poláček and Siegel, let us first presume the megavielbein can be gauged to a triangular form,

$$
\mathcal{V}_{M}{ }^{A}=\left(\begin{array}{lll}
\mathrm{x} & 0 & 0 \\
\mathrm{x} & \mathrm{x} & 0 \\
\mathrm{x} & \mathrm{x} & \mathrm{x}
\end{array}\right), \quad \mathcal{V}_{A}{ }^{M}=\left(\begin{array}{ccc}
\mathrm{x} & 0 & 0 \\
\mathrm{x} & \mathrm{x} & 0 \\
\mathrm{x} & \mathrm{x} & \mathrm{x}
\end{array}\right),
$$

where the $x$ 's denote non-vanishing entries. We emphasize that the entries are ordered so that $\nabla_{A}=\left(\nabla_{\underline{a}}, \nabla_{a}, \nabla \underline{a}\right)$. Defining the antisymmetric current $\mathcal{J}_{B A}$ via $\delta \mathcal{V}_{M}{ }^{A}=\mathcal{V}_{M}{ }^{B} \mathcal{J}_{B C} \eta^{C A}$, a consistency condition for the above gauge is that $\mathcal{J}_{\underline{b a}}=\mathcal{J}_{\underline{b} a}=0$. This immediately implies that covariant diffeomorphisms (B.3) must be constrained as

$$
\begin{aligned}
& 0=\mathcal{J}_{\underline{b a}}=\nabla_{\underline{b}} \xi_{\underline{a}}-\nabla_{\underline{a}} \xi_{\underline{b}}+\xi_{\underline{c}} f^{\underline{c}} \underline{\underline{b} a}, \\
& 0=\mathcal{J}_{\underline{b} a}=\nabla_{\underline{b}} \xi_{a}-\nabla_{a} \xi_{\underline{b}}+\xi_{\underline{c}} f_{\underline{c} \underline{c} a}+\xi^{c} f_{\underline{b} a c} .
\end{aligned}
$$

These conditions are easily solved by taking

$$
\xi_{\underline{a}}=0, \quad \nabla_{\underline{b}} \xi_{a}=-f_{\underline{b} a}{ }^{c} \xi_{c} .
$$

The first condition has the interpretation that once we have imposed the gauge (B.9), the dual $\widehat{\mathcal{H}}$ transformations associated with $\nabla^{\underline{a}}$ no longer play any role. This is good: we are looking for a minimal way to incorporate the gauging of a group $\widehat{\mathcal{H}}$, and having to accommodate another dual group would be excessive. In the triangular gauge, imposing

\footnotetext{
${ }^{17}$ In their original paper [31], Poláček and Siegel employed just the doubled Lorentz group, and so a number of the $f$ 's we have here actually vanish, in particular $f_{\underline{a}} b^{\underline{c}}$.
} 
$\xi_{\underline{a}}=0$ is equivalent to imposing $\xi_{\underline{m}}=0$. The second condition in (B.11) tells us how $\xi_{a}$ transforms under the $\widehat{\mathcal{H}}$ group, which will amount to a condition on its $y$-dependence.

Let's check that the above conditions are consistent. The closure of two diffeomorphisms leads to

$$
\xi_{12}{ }^{A}:=\left(\mathbb{L}_{\xi_{1}} \xi_{2}\right)^{A}=\xi_{1}{ }^{B} \nabla_{B} \xi_{2}{ }^{A}-\xi_{2}{ }^{B} \nabla_{B} \xi_{1}{ }^{A}+\xi_{2}{ }^{B} \nabla^{A} \xi_{1 B}+\xi_{1}{ }^{B} \xi_{2}{ }^{C} \mathcal{T}_{C B}{ }^{A} .
$$

We must take $\xi_{12 a}$ to vanish. As required, this holds given the conditions we have already imposed:

$$
\xi_{12 \underline{a}}=\xi_{2}{ }^{b} \nabla_{\underline{a}} \xi_{1 b}+\xi_{1}{ }^{b} \xi_{2}{ }^{c} \mathcal{T}_{c b \underline{a}}=-\xi_{2}{ }^{b} f_{\underline{a} b}{ }^{c} \xi_{1 c}+\xi_{1}{ }^{b} \xi_{2}{ }^{c} f_{\underline{a} c b}=0 .
$$

We also should check that the second condition (B.11) satisfies integrability:

$$
\left[\nabla_{\underline{a}}, \nabla_{\underline{b}}\right] \xi^{c}=-\nabla_{\underline{a}} \xi^{d} f_{d \underline{b}}^{c}+\nabla_{\underline{b}} \xi^{d} f_{d \underline{a}}^{c}=\xi^{e} f_{e \underline{a}}^{d} f_{d \underline{b}}^{c}-\xi^{e} f_{e \underline{b}}{ }^{d} f_{d \underline{a}}^{c}=-f_{\underline{a} \underline{b}} \nabla_{\underline{d}} \xi^{c},
$$

with the last equality following from the Jacobi identity.

Now let us assign names to the non-vanishing entries of the megavielbein (B.9). Using orthogonality, these can be parametrized as

$$
\begin{aligned}
& \mathcal{V}_{M}^{B}=\left(\begin{array}{ccc}
K_{\underline{m}^{\underline{a}}} & 0 & 0 \\
0 & \mathcal{V}_{m}^{a} & 0 \\
0 & 0 & K_{\underline{a}} \underline{\underline{m}}
\end{array}\right) \times\left(\begin{array}{ccc}
\delta_{\underline{a}}^{\underline{\underline{b}}} & 0 & 0 \\
h_{a}^{\underline{\underline{b}}} & \delta_{a}^{b} & 0 \\
-p^{\underline{a} \underline{b}}-\frac{1}{2} h^{c \underline{a}} h_{c^{\underline{b}}} & -h^{b \underline{a}} & \delta^{\underline{a}} \underline{\underline{b}}
\end{array}\right), \\
& \mathcal{V}_{B}{ }^{M}=\left(\begin{array}{ccc}
\delta_{\underline{b}} \underline{\underline{a}} & 0 & 0 \\
-h_{b} \underline{\underline{a}} & \delta_{b}{ }^{a} & 0 \\
p^{\underline{\underline{b a}}}-\frac{1}{2} h^{c} \underline{\underline{c}} h_{c^{\underline{a}}} h^{a \underline{b}} & \delta_{\underline{\underline{b}}}^{\underline{\underline{b}}}
\end{array}\right) \times\left(\begin{array}{ccc}
K_{\underline{a}} \underline{\underline{m}} & 0 & 0 \\
0 & \mathcal{V}_{a}{ }^{m} & 0 \\
0 & 0 & K_{\underline{m}^{\underline{a}}}
\end{array}\right),
\end{aligned}
$$

where we give both the megavielbein and its inverse for convenience. The element $\mathcal{V}_{m}{ }^{a}$ will be the usual doubled vielbein and $h_{a} \underline{\underline{b}}$ will be the $\widehat{\mathcal{H}}$-connection. The field $p^{\underline{a b}}$ is an antisymmetric tensor, which we call the Poláček-Siegel (PS) field; it transforms non-linearly under the $\widehat{\mathcal{H}}$ gauge group and will allow us to build $\widehat{\mathcal{H}}$-curvatures.

The element $K_{\underline{\underline{m}}} \underline{\underline{a}}$ (with inverse $K_{\underline{a}} \underline{\underline{m}}$ ) will turn out to not play a physical role and will drop out of all relevant formulae. In [31], this field (along with a piece of $\mathcal{V}_{m}{ }^{a}$ ) dresses $\partial_{M}$ to become their background $D_{M}$ derivatives. Here we have found it simpler to exhibit it explicitly and just ensure that it drops out. We should emphasize that it is perfectly possible to impose the gauge where $K_{\underline{\underline{m}}}$ is set to some fixed coordinate-dependent value. This would involve imposing $\nabla_{\underline{b}} \xi^{\underline{a}}=-\overline{\xi^{\underline{c}}} f_{\underline{c} \underline{\underline{b}}}$. However, this is not strictly necessary, so we will avoid doing that here.

In the triangular gauge, the various derivatives decompose as follows:

$$
\begin{aligned}
& \nabla_{\underline{a}}=K_{\underline{a}}^{\underline{m}} \partial_{\underline{m}}, \\
& \nabla_{a}=\mathcal{V}_{a}{ }^{m} \partial_{m}-h_{a} \underline{\underline{b}} \nabla_{\underline{b}}, \\
& \nabla^{\underline{a}}=\left(p^{\underline{a} \underline{b}}-\frac{1}{2} h^{m \underline{a}} h_{m^{\underline{b}}}\right) \nabla_{\underline{b}}+h^{b \underline{a}} \mathcal{V}_{b}{ }^{m} \partial_{m}+K_{\underline{m}}{ }^{\underline{a}} \partial^{\underline{m}} .
\end{aligned}
$$

In the expression for $\nabla^{a}$, the last term can be dropped because we will always assume that no field depends on $\tilde{y}_{m}$. Each of these operators will turn out to be covariant. This is more 
or less obvious for the first two, assuming that they will behave as the $\widehat{\mathcal{H}}$ generator and the $\widehat{\mathcal{H}}$-covariant derivative, respectively. This is less obvious for the third operator, but this will turn out to also be true by virtue of the section condition.

Now let us show how the megavielbein (B.15) solves the torsion constraints. It is easy to see that the conditions $\mathcal{T}_{\underline{c b a}}=\mathcal{T}_{\underline{c} b a}=0$ hold automatically in the triangular gauge. The torsions that are fixed to constants turn out to constrain the $y^{\underline{m}}$ dependence of the various fields:

$$
\begin{aligned}
& \mathcal{T}_{\underline{c} \underline{b}} \underline{a}=f_{\underline{c b}} \underline{a} \quad \Longrightarrow \quad 2 \nabla_{[\underline{c}} K_{\underline{b}]^{\underline{m}}} K_{\underline{\underline{m}}} \underline{\underline{a}}=-f_{\underline{c b}} \underline{a}, \\
& \mathcal{T}_{\underline{c} b a}=f_{\underline{c} b a} \quad \Longrightarrow \quad \nabla_{\underline{c}} \mathcal{V}_{b}{ }^{m}=-f_{\underline{c} b}{ }^{a} \mathcal{V}_{a}{ }^{m} \text {, } \\
& \mathcal{T}_{c \underline{b}} \underline{\underline{a}}=f_{c \underline{b}} \underline{a} \quad \Longrightarrow \quad \nabla_{\underline{b}} h_{m} \underline{\underline{a}}=-h_{m}{ }^{\underline{c}} f_{\underline{c} \underline{\underline{b}}} \underline{a}-\mathcal{V}_{m}{ }^{c} f_{c \underline{b}} \underline{a}+K_{\underline{b}} \underline{\underline{n}} \partial_{m} K_{\underline{\underline{n}}} \underline{\underline{a}}, \\
& \mathcal{T}_{\underline{c}}^{\underline{b a}}=f_{\underline{\underline{c}}}^{\underline{b a}} \quad \Longrightarrow \quad \nabla_{\underline{c}} p^{\underline{b a}}=-f_{\underline{\underline{c}} \underline{b a}}-h^{d[\underline{b}} f_{d \underline{c}} \underline{a}+2 p^{\underline{d}[\underline{b}} f_{\underline{d c}}^{\underline{a}]}-h^{m[\underline{b}} K_{\underline{\underline{c}} \underline{\underline{n}}} \partial_{m} K_{\underline{\underline{n}}} \underline{\underline{a}} \text {. }
\end{aligned}
$$

The remaining torsion tensors are field-dependent and lead to curvatures. The standard $\widehat{\mathcal{H}}$-covariant torsion tensor of DFT is

$$
\mathcal{T}_{c b a}=-3 \nabla_{[c} \mathcal{V}_{b}{ }^{m} \mathcal{V}_{m a]}, \quad \nabla_{c} \mathcal{V}_{b}{ }^{m}:=\mathcal{V}_{c}{ }^{n} \partial_{n} \mathcal{V}_{b}{ }^{m}+h_{c}{ }^{\underline{c}} f_{\underline{c}}{ }^{a} \mathcal{V}_{a}{ }^{m},
$$

where we have used (B.17b) in the definition of $\nabla_{c} \mathcal{V}_{b}{ }^{m}$. The $\widehat{\mathcal{H}}$-curvature is $\mathcal{T}_{c b}$, which is given by

$$
\begin{aligned}
\mathcal{T}_{c b} \underline{\underline{a}}= & D_{c} h_{b} \underline{\underline{a}}-D_{b} h_{c} \underline{\underline{a}}+\mathcal{F}_{c b}{ }^{d} h_{d^{\underline{a}}}-h_{b} \underline{\underline{b}} h_{c} \underline{\underline{c}} f_{c \underline{c} \underline{\underline{a}}}+h_{c} \underline{\underline{c}} f_{b \underline{\underline{c}} \underline{\underline{a}}} \underline{\underline{a}}-h_{b} \underline{\underline{c}} f_{c \underline{\underline{c}} \underline{\underline{a}}} \\
& +\left(p^{\underline{a}}-\frac{1}{2} h^{d \underline{a}} h_{d} \underline{\underline{d}}\right) f_{\underline{d} c b},
\end{aligned}
$$

where $D_{a}:=\mathcal{V}_{a}{ }^{m} \partial_{m}$ and $\mathcal{F}_{a b}{ }^{c}=-3 D_{[a} \mathcal{V}_{b}{ }^{m} \mathcal{V}_{m c]}$ is the generalized flux of the DFT vielbein. Here we see that the PS field $p^{\underline{a b}}$ permits the construction of $\mathcal{T}_{c b} \underline{a}$ explicitly. An important consistency condition is that there remains a combination

$$
\mathcal{T}_{a b} \underline{\underline{c}} f_{\underline{c} c d}+\mathcal{T}_{c d^{\underline{c}}} f_{\underline{c} a b}
$$

where the PS field drops out. For the case where $\widehat{\mathcal{H}}$ is just the doubled Lorentz group, this is the symmetrized Riemann tensor that one can construct. That the PS field permits the direct construction of $T_{a b} \underline{\underline{c}}$ was the key observation of [31].

Finally, we give the remaining invariant tensors. The tensor $\mathcal{T}_{c}^{\underline{b a}}$ is the covariantized gradient of the PS field,

$$
\begin{aligned}
& \mathcal{T}_{c}^{\underline{b a}}=\left[-D_{c} p^{\underline{b a}}-2 h_{c} \underline{\underline{c}} p^{\underline{b d}} f_{\underline{d c}}{ }^{\underline{a}}+2 p^{\underline{b d}} f_{c \underline{d}^{\underline{a}}}{ }^{\underline{a}}\right. \\
& +h^{d \underline{b}} D_{c} h_{d} \underline{\underline{a}}-2 h^{d \underline{b}} D_{d} h_{c}{ }^{\underline{a}}+\mathcal{F}_{c}{ }^{b a} h_{b}{ }^{\underline{b}} h_{a^{\underline{a}}}
\end{aligned}
$$

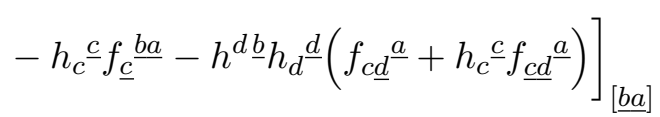

where $\underline{b a}$ are antisymmetrized. Similarly, $\mathcal{T} \underline{c b a}$ is the antisymmetric $\nabla^{\underline{c}}$ derivative of $p^{\underline{b a}}$. Expanding out all terms we find

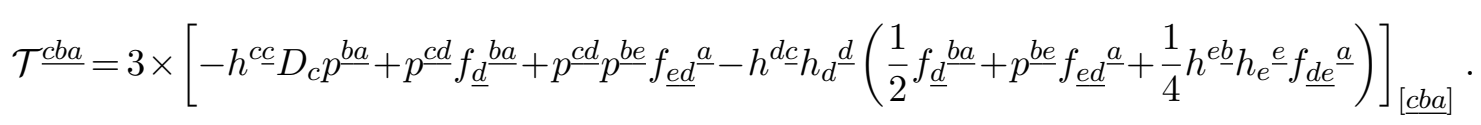


This condition actually follows from the simpler result that $\mathcal{T} \underline{p n m}=0$ from the section condition.

We have not yet specified how the various components of the megavielbein transform. For that, we return to the conventional form of doubled diffeomorphisms (B.2) on the megavielbein. First, we check that the zero elements remain zero,

$$
\begin{aligned}
& 0=\delta \mathcal{V}_{\underline{m}}^{a}=\left(\partial_{\underline{m}} \xi^{n}-\partial^{n} \xi_{\underline{m}}\right) \mathcal{V}_{n}{ }^{a}+\left(\partial_{\underline{m}} \xi_{\underline{n}}-\partial_{\underline{n}} \xi_{\underline{m}}\right) \mathcal{V}^{\underline{n} a}, \\
& 0=\delta \mathcal{V}_{\underline{m a}}=\left(\partial_{\underline{m}} \xi_{\underline{n}}-\partial_{\underline{n}} \xi_{\underline{m}}\right) \mathcal{V}^{\underline{n}} \underline{a} .
\end{aligned}
$$

These are consistent if we choose $\xi_{\underline{m}}=0$ and $\partial_{\underline{m}} \xi^{n}=0$, which follow from (B.11) and (B.17b). The DFT vielbein transforms, using (B.17b), as

$$
\delta \mathcal{V}_{m}^{a}=\mathbb{L}_{\xi} \mathcal{V}_{m}^{a}+\mathcal{V}_{m}^{b} \Lambda^{\underline{c}} f_{\underline{c} b}^{a}, \quad \Lambda^{\underline{a}}:=\xi^{\underline{m}} K_{\underline{m}^{\underline{a}}}{ }^{a} .
$$

The $\widehat{\mathcal{H}}$ connection transforms, using (B.17c), as

$$
\delta h_{m}^{\underline{a}}=\mathbb{L}_{\xi} h_{m} \underline{\underline{a}}+\partial_{m} \Lambda^{\underline{a}}+h_{m} \underline{\underline{b}} \Lambda^{\underline{c}} f_{\underline{c} \underline{\underline{a}}}^{\underline{a}}+\mathcal{V}_{m}{ }^{b} \Lambda^{\underline{c}} f_{\underline{c} b^{\underline{a}}}^{\underline{a}} .
$$

Finally, the PS field transforms as

$$
\delta p^{\underline{a b}}=\xi^{m} \partial_{m} p^{\underline{a b}}-\Lambda^{\underline{c}} f_{\underline{c}}^{\underline{a b}}-2 \Lambda^{\underline{c}} p^{\underline{d}[\underline{a}} f_{\underline{c d}} \underline{b}-h^{m[\underline{a}} \partial_{m} \Lambda^{\underline{b}]}-\Lambda^{\underline{c}} h^{c[\underline{a}} f_{c \underline{c}}^{\underline{b}]} .
$$

In writing the transformations this way, we have eliminated all of the $y$ derivatives, so the $y$-dependence can be thought of as merely a trick to arrive at these transformation rules. One could in principle eliminate the $y$ dependence explicitly by introducing $y$-dependent twist matrices for the various connections in order to solve the conditions (B.17) directly. However, it is simpler instead merely to verify that the algebra of $\widehat{\mathcal{H}}$ transformations closes on all of the fields above.

There are a few more issues we have not yet explicitly addressed. First, we mention how the torsions and curvatures each transform. From the extended Bianchi identity, one can show that

$$
\left.\delta_{\Lambda} \mathcal{T}_{b c d}:=\Lambda^{\underline{a}} \nabla_{\underline{a}} \mathcal{T}_{b c d}=-3 \Lambda^{\underline{a}}\left(f_{\underline{a}[b \mid}{ }^{e} \mathcal{T}_{e \mid c d]}+f_{\underline{a}[b \mid} \underline{e}^{\underline{e}} f_{\underline{e}} \mid c d\right]\right)
$$

This follows as well from the explicit definition of the torsion tensor. If we assume that there is a background constant value for the torsion, $\stackrel{\circ}{\mathcal{T}} a b c=f_{a b c}$, for which (B.8) corresponds to a closed Lie algebra, then the above transformation can be written

$$
\delta_{\Lambda} \mathcal{T}_{b c d}=-3 \Lambda^{\underline{a}} f_{\underline{a}[b \mid} e\left(\mathcal{T}_{e \mid c d]}-\stackrel{\circ}{\mathcal{T}}_{e \mid c d]}\right) \equiv-3 \Lambda^{\underline{a}} f_{\underline{a}[b \mid}^{e} \Delta \mathcal{T}_{e \mid c d]}
$$

with $\Delta \mathcal{T}$ denoting the deviation from the background.

The Bianchi identity for the torsion tensor becomes

$$
\left.0=4 \nabla_{[a} \mathcal{T}_{b c d]}+3 \mathcal{T}_{[a b}{ }^{e} \mathcal{T}_{c d] e}+6 \mathcal{R}_{[a b} f_{\underline{e}} f d\right]
$$

where we have written the $\widehat{\mathcal{H}}$-curvature as $\mathcal{R}_{a b} \underline{c}:=\mathcal{T}_{a b}$ 드 to improve readability. Observe that the PS field $p \underline{a b}$ drops out of the combination of curvatures appearing above. In addition, 
due to the projection with $f_{\underline{a c d}}$, only some of the $\widehat{\mathcal{H}}$-curvatures play a role in the Bianchi identity. Naturally these are those with nonzero $f_{\underline{a} c d}$ as only these $\widehat{\mathcal{H}}$ connections appear in the torsion tensor.

The $\widehat{\mathcal{H}}$-curvature itself transforms as

$$
\begin{aligned}
& \delta_{\Lambda} \mathcal{R}_{b c} \underline{\underline{d}}=-\Lambda^{\underline{a}}\left(2 \mathcal{T}_{\underline{a}[b}{ }^{E} \mathcal{T}_{c]} \underline{d}_{E}+\mathcal{T}_{b c}{ }^{E} \underline{\mathcal{T}}_{\underline{\underline{a}}}{ }^{\underline{d}}{ }\right) \\
& =-\Lambda \underline{a}\left(f_{\underline{a} b}^{e} \Delta \mathcal{R}_{e c} \underline{d}^{\underline{a}}+f_{\underline{a} c}{ }^{e} \Delta \mathcal{R}_{b e^{\underline{d}}}+\Delta \mathcal{R}_{b c} \underline{e}_{\underline{e a}}^{\underline{d}}+\Delta \mathcal{T}_{b c}{ }^{e} f_{e \underline{a}} \underline{d}\right),
\end{aligned}
$$

where $\Delta \mathcal{R}$ denotes the deviation of $\mathcal{R}$ from its background value. (We allow for this

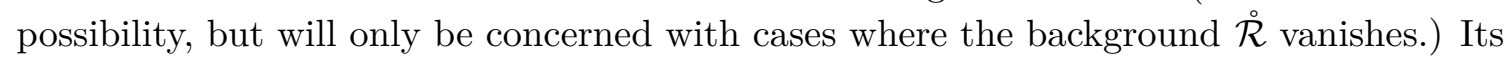
Bianchi identity is a bit more complicated:

$$
3 \nabla_{a} \mathcal{R}_{b c} \underline{\underline{d}}-\nabla \underline{\mathcal{T}}_{a b c}+3 \mathcal{T}_{a b}{ }^{e} \mathcal{R}_{e c} \underline{\underline{d}}+3 \mathcal{R}_{a b} \underline{e}_{\underline{\underline{e}}} \underline{\underline{d}}^{\underline{d}}+\left.3 \mathcal{R}_{c} \underline{\underline{d e}} f_{\underline{\underline{e}} a b}\right|_{[a b c]}=0 .
$$

This is quite an awkward identity as $\nabla \underline{a}$ involves a naked connection.

The additional gauge transformations are

$$
\begin{aligned}
& \delta_{\Lambda} \mathcal{R}_{b} \underline{\underline{c d}}=\left.\Lambda \underline{a}\left(-f_{\underline{a} b}{ }^{e} \Delta \mathcal{R}_{e} \underline{\underline{c d}}+2 \Delta \mathcal{R}_{b} \underline{\underline{e d}} f_{\underline{e a}}^{\underline{c}}+2 f_{\underline{a}}^{\underline{c e}} \Delta \mathcal{R}_{e b} \underline{\underline{d}}\right)\right|_{[\underline{c d}]}, \\
& \delta_{\Lambda} \mathcal{R}^{\underline{b c d}}=-\left.3 \Lambda^{\underline{a}}\left(f_{e \underline{\underline{a}}} \mathcal{R}^{e \underline{c d}}+f_{\underline{a}}^{\underline{b e}} f_{\underline{\underline{e}}}^{\underline{c d}}+\mathcal{R} \frac{c d e}{f_{\underline{e a}}}\right)\right|_{[\underline{b c d}]},
\end{aligned}
$$

and the remaining Bianchi identities are

$$
\begin{aligned}
& 0=2 \nabla_{a} \mathcal{R}_{b}{ }^{\underline{c d}}+2 \nabla^{\underline{c}} \mathcal{R}_{a b} \underline{\underline{d}}+T_{a b}{ }^{e} \mathcal{R}{ }^{\underline{c d}}+\mathcal{R}_{a b}{ }^{\underline{e}} f_{\underline{e}}^{\underline{c d}}+\mathcal{R}^{\underline{c d e}} f_{\underline{e} a b} \\
& -2 \mathcal{R}_{a}^{e}{ }_{a}^{\underline{c}} \mathcal{R}_{e b} \underline{\underline{d}}-2 \mathcal{R}_{a} \underline{\underline{c e}} f_{\underline{e} b^{\underline{d}}}-\left.2 \mathcal{R}_{b} \underline{\underline{d e}} f_{\underline{\underline{e}} a}\right|_{[\underline{c} \underline{c}]} \\
& 0=\nabla_{a} \mathcal{R}^{\underline{b c d}}-3 \nabla^{\underline{b}} \mathcal{R}_{a} \underline{\underline{c d}}+3 \mathcal{R}^{e}{ }_{a}^{\underline{b}} \mathcal{R}_{e^{\underline{c d}}}+3 \mathcal{R}_{a}{ }^{\underline{b e}} f_{\underline{\underline{e}}}^{\underline{c d}}+\left.3 f_{\underline{\underline{e}} a} \underline{\underline{b}} \mathcal{R} \underline{\underline{e c d}}\right|_{[\underline{b c d]}} .
\end{aligned}
$$

There is actually one more feature of DFT that we have not discussed: the dilaton. In the enlarged Poláček-Siegel theory, the dilaton is a scalar $\widehat{\Phi}$ that transforms as

$$
\delta \widehat{\Phi}=\partial_{M}\left(\xi^{M} \widehat{\Phi}\right)=\partial_{m}\left(\xi^{m} \widehat{\Phi}\right)+\partial_{\underline{m}}\left(\xi^{\underline{m}} \widehat{\Phi}\right) .
$$

Its curvature $\widehat{\mathcal{T}}_{A}$ and Bianchi identity are given by

$$
\widehat{\mathcal{T}}_{A}=\nabla_{A} \log \widehat{\Phi}+\partial_{M} \mathcal{V}_{A}{ }^{M}, \quad 2 \nabla_{[A} \widehat{\mathcal{T}}_{B]}=-\mathcal{T}_{A B}{ }^{C} \widehat{\mathcal{T}}_{C}-\nabla^{C} \mathcal{T}_{C A B}
$$

The actual dilaton we want differs from this dilaton as

$$
\log \Phi=\log \widehat{\Phi}-\log \operatorname{det} K_{\underline{m}^{\underline{a}}} .
$$

It follows that

$$
\begin{aligned}
\widehat{\mathcal{T}}_{a} & =\mathcal{T}_{a}+f_{a \underline{b}} \underline{\underline{b}} \\
\widehat{\mathcal{T}}_{\underline{a}} & =\nabla_{\underline{a}} \log \Phi+f_{\underline{a}^{\underline{b}}} \underline{\underline{a}} \\
\widehat{\mathcal{T}}^{\underline{a}} & =D^{b} h_{b} \underline{\underline{a}}+\mathcal{F}^{b} h_{b} \underline{\underline{a}}+h^{d \underline{b}} f_{d \underline{\underline{b}}} \underline{\underline{a}}+p^{\underline{b c}} f_{\underline{c} \underline{a}} \underline{a}+f_{\underline{b}} \underline{b a}+\left(p^{\underline{a} \underline{b}}-\frac{1}{2} h^{d \underline{a}} h_{d^{\underline{b}}}\right) \nabla_{\underline{b}} \log \Phi
\end{aligned}
$$


where we have defined

$$
\mathcal{F}_{a}:=D_{a} \log \Phi+\partial_{m} \mathcal{V}_{a}{ }^{m}, \quad \mathcal{T}_{a}:=\nabla_{a} \log \Phi+\nabla_{m} \mathcal{V}_{a}{ }^{m}
$$

Evidently, $\widehat{\mathcal{T}}_{a}$ is merely a constant shift from $\mathcal{T}_{a}$. Assuming the dilaton transforms homogeneously, so that $\nabla_{\underline{a}} \log \Phi$ is a constant (or zero), we find that $\widehat{\mathcal{T}}_{\underline{a}}$ is also a constant. Finally, the last curvature $\widehat{\mathcal{T}} \underline{a}$ is partly related to the tensor we previously called $\widehat{\mathcal{R}}_{\hat{a} \hat{b}}$ in (2.29) for the case where the $\widehat{\mathcal{H}}$ group involved just the doubled Lorentz group.

The Bianchi identity now decomposes to

$$
2 \nabla_{[a} \mathcal{T}_{b]}=-\mathcal{T}_{a b}{ }^{c} \mathcal{T}_{c}-\nabla^{c} \mathcal{T}_{c a b}-\mathcal{R}_{a b}{ }^{\underline{c}} \nabla_{\underline{c}} \log \Phi-\mathcal{R}_{a}{ }^{d \underline{c}} f_{\underline{c} d b}+\mathcal{R}_{b}{ }^{d \underline{c}} f_{\underline{c} d a}-\widehat{\mathcal{T}}^{\underline{c}} f_{\underline{c} a b}
$$

It also follows that $\mathcal{T}_{a}$ transforms under $\widehat{\mathcal{H}}$ transformations as

$$
\delta_{\Lambda} \mathcal{T}_{b}=-\Lambda^{\underline{a}}\left(f_{\underline{a} b}^{c} \mathcal{T}_{c}+f_{\underline{a}} b^{\underline{c}} \mathcal{T}_{\underline{c}}\right) .
$$

\section{Construction of the extended super-Maxwell $1_{\infty}$ algebra}

In this appendix, we detail the construction of a superalgebra which appears to coincide with the $\widehat{\mathcal{H}}_{L}$ tangent space algebra required for $\mathcal{N}=1 \mathrm{DFT}$, introduced in section 4.3. As mentioned in the main body, it arises as the dual of what we call the super-Maxwell $\infty$ algebra. We first discuss what precisely we mean by the super-Maxwell $\infty$ algebra, and then review a construction, based on the notion of a local Lie superalgebra [40], that leads to both the full superalgebra involving both it and its dual, that we need to construct the connections and curvatures of $\mathcal{N}=1 \mathrm{DFT}$.

\section{C.1 The super-Maxwell $1_{\infty}$ algebra}

Here we detail the construction of the free differential algebra, up through dimension 4 , that extends the $10 \mathrm{D} \mathcal{N}=1$ super-Poincaré algebra

$$
\left\{Q_{\alpha}, Q_{\beta}\right\}=\left(\gamma^{a}\right)_{\alpha \beta} P_{a} .
$$

$Q_{\alpha}$ and $P_{a}$ are assigned their usual engineering dimensions of $+\frac{1}{2}$ and +1 . Relative to the main body of the text, we have eliminated the constant $-\kappa$ to simplify formulae, but will restore it later.

Let us now construct the free Lie algebra that extends the super-Poincaré algebra. Following [32, 33], we denote this the super-Maxwell $\infty$ algebra. Denote the generators encountered in this algebra by $Y$ with various subscripts and superscripts. We use a shorthand notation where, for example, $Y_{A, B}$ denotes $\left[P_{A}, P_{B}\right]$ and $Y_{A, B, C}$ denotes $\left[P_{A},\left[P_{B}, P_{C}\right]\right]$ where $P_{A}=\left(Q_{\alpha}, P^{a}\right)$. In this way, (C.1) could be denoted $Y_{\alpha, \beta}=\left(\gamma^{a}\right)_{\alpha \beta} Y_{a}$.

Let's begin by analyzing the generator at dimension $\frac{3}{2}$. The Bianchi identity for three $Q$ 's is

$$
Y_{(\alpha, \beta, \gamma)}=\left(\gamma^{b}\right)_{(\beta \gamma} Y_{\alpha), b}=0
$$


This is solved by taking

$$
\left[Q_{\alpha}, P_{b}\right] \equiv Y_{\alpha, b}=\left(\gamma_{b}\right)_{\alpha \beta} \tilde{Q}^{\beta}
$$

for some fermionic operator $\tilde{Q}^{\alpha}$ at dimension $\frac{3}{2}$. In keeping with our DFT motivation, we will consider $\tilde{Q}$ together with $Q$ and $P$ as our super $P_{A}$ operators.

Here we pause and make an important observation. In analyzing the algebra, it is extremely helpful to remember that the algebra (C.1) is obeyed by the covariant superspace derivatives that describe the super-Yang-Mills algebra in superspace. In that case, the operator $\tilde{Q}^{\alpha}$ encountered above is just $\boldsymbol{\lambda}^{\alpha}$, the gaugino superfield. We will find that other generators we encounter at higher dimension admit a similar simple interpretation.

At dimension 2, a new generator can be introduced as

$$
Y_{a b}:=Y_{a, b} \equiv\left[P_{a}, P_{b}\right] .
$$

In the super-Yang-Mills case, this is just the non-abelian field strength $\boldsymbol{F}_{a b}$. The Jacobi identity at this dimension reads

$$
\begin{aligned}
0 & =\left\{Q_{\alpha},\left[Q_{\beta}, P_{b}\right]\right\}+\left\{Q_{\beta},\left[Q_{\alpha}, P_{b}\right]\right\}+\left[P_{b},\left\{Q_{\alpha}, Q_{\beta}\right\}\right] \\
& =Y_{\alpha, \beta, b}+Y_{\beta, \alpha, b}+Y_{b, \alpha, \beta} \\
& =\left(\gamma_{b}\right)_{\beta \gamma} Y_{\alpha,}{ }^{\gamma}+\left(\gamma_{b}\right)_{\alpha \gamma} Y_{\beta},^{\gamma}+\left(\gamma^{c}\right)_{\alpha \beta} Y_{b c}
\end{aligned}
$$

This is solved by taking

$$
Y_{\alpha,}^{\beta}=\frac{1}{4}\left(\gamma^{a b}\right)_{\alpha}^{\beta} Y_{a b}
$$

In the case of the super-Yang-Mills algebra, this is the condition that the spinor derivative of the gaugino superfield is the field strength, i.e. $\mathcal{D}_{\alpha} \boldsymbol{\lambda}^{\beta} \propto\left(\gamma^{a b}\right)_{\alpha}{ }^{\beta} \boldsymbol{F}_{a b}$.

At dimension $\frac{5}{2}$, a new generator may be denoted

$$
Y_{b}^{\alpha}:=Y_{b}{ }^{\alpha} \equiv\left[P_{b}, \tilde{Q}^{\alpha}\right] .
$$

This will turn out to be the only generator at this dimension. The commutator of $Q_{\alpha}$ with $Y_{b c}$ just reproduces this generator:

$$
\left[Q_{\alpha}, Y_{b c}\right] \equiv Y_{\alpha, b c}=\left(\gamma_{b}\right)_{\alpha \gamma} Y_{c}^{\gamma}+\left(\gamma_{c}\right)_{\alpha \gamma} Y_{b}^{\gamma}=-2\left(\gamma_{[b}\right)_{\alpha \gamma} Y_{c]}^{\gamma}
$$

Actually, this generator turns out to carry a constraint: its spin- $1 / 2$ part, corresponding to the $\gamma$ trace, vanishes. This follows from the Jacobi identity:

$$
\begin{aligned}
0 & =\left[Q_{\alpha},\left\{Q_{\beta}, Q^{\gamma}\right\}\right]+\left[Q_{\beta},\left\{Q_{\alpha}, Q^{\gamma}\right\}\right]+\left[Q^{\gamma},\left\{Q_{\alpha}, Q_{\beta}\right\}\right] \\
& =2 Y_{(\alpha, \beta)}{ }^{\gamma}+Y_{, \alpha, \beta}^{\gamma}=\frac{1}{2}\left(\gamma^{a b}\right)_{(\alpha}^{\gamma} Y_{\beta), a b}-\left(\gamma^{c}\right)_{\alpha \beta} Y_{c}^{\gamma} \\
& =-\frac{1}{2}\left(\gamma_{a}\right)_{\alpha \beta} Y_{b}^{\delta}\left(\gamma^{b} \gamma^{a}\right)_{\delta}^{\gamma}+\delta_{\alpha}^{\gamma} Y_{b}^{\delta}\left(\gamma_{b}\right)_{\beta \delta}
\end{aligned}
$$

This vanishes only if $Y_{b}{ }^{\beta}$ is $\gamma$-traceless. In the super-Yang-Mills algebra, this condition can be interpreted as the gaugino field equation. 
At dimension 3, we encounter

$$
Y_{a, b c} \equiv\left[P_{a}, Y_{b c}\right]=\left[P_{a},\left[P_{b}, P_{c}\right]\right]
$$

The antisymmetric part of this vanishes, as a consequence of the Jacobi identity. In the super-Yang-Mills algebra, this is $\mathcal{D}_{a} \boldsymbol{F}_{b c}$, and the vanishing of its totally antisymmetric part is the Bianchi identity. Other possible generators we encounter are

$$
Y_{\alpha, b}^{\beta} \equiv\left\{Q_{\alpha}, Y_{b}^{\beta}\right\}, \quad Y^{\alpha \beta}:=\left\{Q^{\alpha}, Q^{\beta}\right\} .
$$

From the Jacobi identity involving $Q, P$, and $\tilde{Q}$, it is easy to show that the first of these is given in terms of the other two:

$$
Y_{\alpha, b}^{\beta}=\left(\gamma_{b}\right)_{\alpha \gamma} Y^{\gamma \beta}+\frac{1}{4}\left(\gamma^{c d}\right)_{\alpha}^{\beta} Y_{b, c d}
$$

Actually, because the left-hand side is $\gamma$-traceless in its last two indices, a constraint must be satisfied:

$$
Y_{, b a}^{b}=-\left(\gamma_{a}\right)_{\alpha \beta} Y^{\alpha \beta}
$$

In the super Yang-Mills algebra, this is the equation of motion for the gauge connection, setting the divergence of the field strength tensor equal to the gaugino bilinear $\left\{\boldsymbol{\lambda}^{\alpha}, \boldsymbol{\lambda}^{\beta}\right\}$. The Jacobi identity involving two $Q$ 's and $Y_{a b}$ tells us no new information. For later use, it will be helpful to decompose $Y_{a, b c}$ into its irreducible (traceless) hook representation $Y_{a \mid b c}$ and the trace:

$$
\begin{aligned}
Y_{a, b c} & =Y_{a \mid b c}+\frac{1}{9} \eta_{a b} Y_{, d c}^{d}-\frac{1}{9} \eta_{a c} Y_{, d b}^{d} \\
& =Y_{a \mid b c}-\frac{2}{9} \eta_{a[b}\left(\gamma_{c]}\right)_{\alpha \beta} Y^{\alpha \beta} .
\end{aligned}
$$

At this level, $Y_{a \mid b c}$ and $Y^{\alpha \beta}$ may be considered the independent operators.

At dimension $\frac{7}{2}$, we identify

$$
Y_{a, b}^{\beta} \equiv\left[P_{a}, Y_{b}^{\beta}\right]
$$

By construction it is $\gamma$-traceless on $b \beta$. This turns out to be the only operator present at this dimension as other commutators just lead ultimately to this one:

$$
\begin{aligned}
Y^{\alpha}{ }_{, a b} \equiv\left[\tilde{Q}^{\alpha}, Y_{a b}\right] & =-2 Y_{[a, b]}^{\alpha}, \\
Y_{\alpha,}{ }^{\beta \gamma} \equiv\left[Q_{\alpha}, Y^{\beta \gamma}\right] & =\left(\gamma^{a b}\right)_{\alpha}{ }^{(\beta} Y_{a, b}{ }^{\gamma)}, \\
Y_{\alpha, b, c d} \equiv\left[Q_{\alpha}, Y_{b, c d}\right] & =-2\left(\gamma_{b}\right)_{\alpha \gamma} Y_{[c, d]}^{\gamma}-\left(\gamma_{c}\right)_{\alpha \gamma} Y_{b, d}{ }^{\gamma}+\left(\gamma_{d}\right)_{\alpha \gamma} Y_{b, c}{ }^{\gamma} .
\end{aligned}
$$

As a check, one can verify that the last two equations are consistent with (C.13), with no assumption on $Y_{a, b}{ }^{\beta}$ aside from it being $\gamma$-traceless in its last two indices. In the super Yang-Mills case, this object is two covariant derivatives of the gaugino superfield $\mathcal{D}_{a} \mathcal{D}_{b} \lambda^{\beta}$. One might expect that instead we should deal with the traceless and $\gamma$-traceless projection of $\mathcal{D}_{(a} \mathcal{D}_{b)} \boldsymbol{\lambda}^{\beta}$ and separately the operator $\left[\boldsymbol{F}_{a b}, \boldsymbol{\lambda}^{\beta}\right]$. However, these two representations 
precisely match the former, i.e. $10000 \times 10010=20010+01000 \times 00010$, so we will remain with the former.

At dimension 4, we identify the following two operators:

$$
Y_{, b}^{\alpha} \equiv\left[\tilde{Q}^{\alpha}, Y_{b}^{\beta}\right], \quad Y_{a, b, c d} \equiv\left[P_{a}, Y_{b, c d}\right] .
$$

In the super Yang-Mills algebra, these are $\left\{\boldsymbol{\lambda}^{\alpha}, \mathcal{D}_{b} \boldsymbol{\lambda}^{\beta}\right\}$ and $\mathcal{D}_{a} \mathcal{D}_{b} \boldsymbol{F}_{c d}$, respectively. Other potential operators are related to these. For example,

$$
\begin{aligned}
& Y_{a,}{ }^{\beta \gamma} \equiv\left[P_{a}, Y^{\beta \gamma}\right]=Y_{, a}^{\beta}{ }_{,{ }^{\gamma}}+Y_{, a}^{\gamma}{ }^{\beta}, \\
& Y_{a b, c d} \equiv\left[Y_{a b}, Y_{c d}\right]=Y_{a, b, c d}-Y_{b, a, c d} .
\end{aligned}
$$

The operator generated by $Q_{\alpha}$ on $Y_{a, b}{ }^{\beta}$ is a bit more involved:

$$
\left\{Q_{\alpha}, Y_{a, b}{ }^{\beta}\right\}=\left(\gamma_{a}\right)_{\alpha \gamma} Y_{, b}^{\gamma}+\left(\gamma_{b}\right)_{\alpha \gamma} Y_{, a}^{\gamma}{ }^{\beta}+\left(\gamma_{b}\right)_{\alpha \gamma} Y_{, a}^{\beta}+\frac{1}{4}\left(\gamma^{c d}\right)_{\alpha}{ }^{\beta} Y_{a, b, c d}
$$

Checking the $\gamma$-traceless condition implies that

$$
Y_{a,{ }_{, b c}}{ }^{b}=-2\left(\gamma_{c}\right)_{\alpha \beta} Y^{\alpha}{ }_{, a}{ }^{\beta} .
$$

This condition is actually just a direct consequence of (C.14), and amounts to

$$
Y_{a, b, c d}=Y_{a, b \mid c d}-\frac{2}{9} \eta_{b c}\left(\gamma_{d}\right)_{\alpha \beta} Y_{, a}^{\alpha}+\frac{2}{9} \eta_{b d}\left(\gamma_{c}\right)_{\alpha \beta} Y_{, a}^{\alpha} .
$$

Analyzing the remaining Jacobi identities at this dimension leads to only one additional constraint. This is best formulated in terms of the reducible operator $Y_{a, b, c d}$, where it reads

$$
Y_{[a, b], c d}+Y_{[c, d], a b}=0 .
$$

In terms of (reducible) Young tableaux, this eliminates the $\square$ representation, leaving the $\square \square$ and $\square$ representations. This identity is obvious for the super Yang-Mills case: $Y_{[a, b], c d}$ is analogous to $\mathcal{D}_{[a} \mathcal{D}_{b]} \boldsymbol{F}_{c d} \propto\left[\boldsymbol{F}_{a b}, \boldsymbol{F}_{c d}\right]$. We solve this constraint by writing

$$
Y_{[a, b], c d}=\frac{1}{2} Y_{a b, c d} .
$$

There is no constraint on $Y_{a b, c d}$ aside from pairwise antisymmetry, $Y_{a b, c d}=-Y_{c d, a b}$. Again, this is obvious from considering the super Yang-Mills case, where it is the commutator of two field strengths.

We would like to identify whatever residual piece of $Y_{a, b \mid c d}$ is independent of either $Y_{a b, c d}$ or $Y^{\alpha}{ }_{, b}^{\beta}$. The $\mathrm{SO}(9,1)$ reps found in $Y_{a, b \mid c d}, Y_{a b, c d}$ and $Y^{\alpha}{ }_{, b}{ }^{\beta}$ are

$$
\begin{aligned}
Y_{a, b \mid c d} & : 01000+10100+20000+02000+21000, \\
Y_{a b, c d} & : 01000+10100, \\
Y^{\alpha}{ }_{, b}^{\beta} & : \underline{01000}+10100+\underline{20000}+00011+10020 .
\end{aligned}
$$

The underlined representations for $Y^{\alpha}{ }_{, b}{ }^{\beta}$ correspond to those that occur in (C.21), so these are the ones that in principle could be related to the corresponding reps in $Y_{a, b \mid c d}$ by 
the constraint (C.22). Meanwhile, this constraint itself decomposes as $00000+00011+$ $02000+20000$. The upshot of this is that of the reps in $Y_{a, b \mid c d}$, only the 21000 is an independent generator. This corresponds to a traceless tensor $Y_{a|b| c d}$, which is symmetric in $a b$, antisymmetric in $c d$, and obeying $Y_{a \mid[b \mid c d]}=0$. The antisymmetric tensor 01000 and the irreducible hook 10100 correspond to $Y_{a b, c d}$; the 20000 is identified with that piece of $Y_{, b}^{\alpha}$; and the 02000 is constrained to vanish. The resulting decomposition can be written

$$
\begin{aligned}
Y_{a, b, c d}=[ & Y_{a|b| c d}+\frac{1}{2} Y_{a b, c d}+\frac{1}{2} Y_{a c, b d} \\
& +\eta_{a c}\left(\frac{1}{8} Y_{b}{ }^{e}, d e-\frac{1}{30}\left(\gamma_{b}\right)_{\alpha \beta} Y^{\alpha}{ }_{, d^{\beta}}-\frac{11}{30}\left(\gamma_{d}\right)_{\alpha \beta} Y_{,{ }^{\beta}}{ }^{\beta}\right) \\
& +\eta_{b c}\left(\frac{1}{8} Y_{a}^{e}{ }_{, d e}-\frac{1}{30}\left(\gamma_{a}\right)_{\alpha \beta} Y^{\alpha}{ }_{, d}{ }^{\beta}-\frac{11}{30}\left(\gamma_{d}\right)_{\alpha \beta} Y_{, a}^{\alpha}\right) \\
& \left.+\eta_{a b}\left(\frac{1}{8} Y_{c}{ }^{e}, d e+\frac{1}{3}\left(\gamma_{c}\right)_{\alpha \beta} Y^{\alpha}{ }_{, d}{ }^{\beta}\right)\right]_{[c d]}
\end{aligned}
$$

Of course, what we really need is the commutator of $P_{a}$ with the independent generator $Y_{b \mid c d}$, which we denote

$$
\begin{aligned}
{\left[P_{a}, Y_{b \mid c d}\right]=Y_{a, b \mid c d}=[} & Y_{a|b| c d}+\frac{1}{2} Y_{a b, c d}+\frac{1}{2} Y_{a c, b d} \\
& +\eta_{a c}\left(\frac{1}{8} Y_{b}{ }^{e}, d e-\frac{1}{30}\left(\gamma_{b}\right)_{\alpha \beta} Y_{,{ }^{\prime}{ }^{\beta}}-\frac{11}{30}\left(\gamma_{d}\right)_{\alpha \beta} Y_{,{ }^{\beta}}^{\alpha}\right) \\
& +\eta_{b c}\left(\frac{1}{8} Y_{a}{ }^{e}, d e-\frac{1}{30}\left(\gamma_{a}\right)_{\alpha \beta} Y_{, d^{\beta}}^{\alpha}+\frac{7}{90}\left(\gamma_{d}\right)_{\alpha \beta} Y_{, a}^{\alpha}\right) \\
& \left.+\eta_{a b}\left(\frac{1}{8} Y_{c}{ }^{e}, d e+\frac{1}{3}\left(\gamma_{c}\right)_{\alpha \beta} Y_{, d}^{\alpha}\right)\right]_{[c d]}
\end{aligned}
$$

This can also be written compactly as

$$
Y_{a, b \mid c d}=Y_{a|b| c d}+\left[\frac{3}{4} Y_{a b, c d}+\frac{3}{8} \eta_{a c} Y_{b}^{e}, d e+\frac{3}{10} \eta_{a c}\left(\gamma_{b}\right)_{\alpha \beta} Y_{, d}^{\alpha}-\frac{7}{10} \eta_{a c}\left(\gamma_{d}\right)_{\alpha \beta} Y_{, b}^{\alpha}\right]_{b \mid c d}
$$

where the projection onto the irreducible hook is implied by the brackets.

This was rather involved, so let's pause to emphasize that at dimension 4 , there are three independent generators:

$$
Y_{, b}^{\alpha}, \quad Y_{a b, c d}, \quad Y_{a|b| c d} .
$$

The first is $\gamma$-traceless in its last two indices. The second is pairwise antisymmetric. Both of these are reducible. The third is irreducible, corresponding to the 21000 representation. In the super-Yang-Mills case, the 21000 corresponds to the irreducible representation involving two symmetrized covariant derivatives of the field strength, where the divergences and Bianchi identity terms have been projected out.

We will not exhaustively analyze the generators at dimension $\frac{9}{2}$, except to say that by comparing with the super-Yang-Mills case, we expect to encounter four. The first, 
$Y^{\alpha \mid \beta \gamma}$ corresponds to the product of three gauginos, $\left[\boldsymbol{\lambda}^{\alpha},\left\{\boldsymbol{\lambda}^{\beta}, \boldsymbol{\lambda}^{\gamma}\right\}\right]$; it lies in the hook representation because the totally symmetric part vanishes due to the Jacobi identity for the non-abelian gauge group in which the gauginos are valued. The second, $Y_{a b, c}{ }^{\gamma}$ corresponds to $\left[\boldsymbol{F}_{a b}, \mathcal{D}_{c} \boldsymbol{\lambda}^{\gamma}\right]$. The third, $Y^{\alpha}{ }_{, b \mid c d}$ corresponds to $\left[\boldsymbol{\lambda}^{\alpha}, \mathcal{D}_{b} \boldsymbol{F}_{c d}\right]$. The fourth is $Y_{(a b c)}{ }^{\gamma}$, which is $\gamma$-traceless and $\eta$-traceless, corresponds to $\mathcal{D}_{(a} \mathcal{D}_{b} \mathcal{D}_{c)} \boldsymbol{\lambda}^{\gamma}$ with the traces removed (as they correspond to the previously mentioned operators).

It is clear from the super-Yang-Mills analogy that this algebra is infinite in extent, although it can be truncated at any level. This ceases to be true when one includes the negative dimension generators of $\widehat{\mathcal{H}}$.

Let us reintroduce factors of $\kappa$ to more easily match our conventions in the main body. This involves replacing

$$
P_{a} \rightarrow-\kappa P_{a}, \quad \tilde{Q}^{\alpha} \rightarrow \kappa^{2} \tilde{Q}^{\alpha}
$$

We keep all defining relations for $Y$ 's formally the same. This means we take

$$
\begin{aligned}
& Y_{a b} \rightarrow \kappa^{2} Y_{a b}, \quad Y_{\alpha}{ }^{\beta} \rightarrow \kappa^{2} Y_{\alpha}{ }^{\beta}, \\
& Y_{b}^{\alpha} \rightarrow-\kappa^{3} Y_{b}^{\alpha}, \quad Y_{\alpha, b c} \rightarrow \kappa^{2} Y_{\alpha, b c}, \\
& Y_{a, b c} \rightarrow-\kappa^{3} Y_{a, b c}, \quad Y_{\alpha, b}^{\beta} \rightarrow-\kappa^{3} Y_{\alpha, b}^{\beta}, \quad Y^{\alpha \beta} \rightarrow \kappa^{4} Y^{\alpha \beta}, \\
& Y_{a, b}{ }^{\beta} \rightarrow \kappa^{4} Y_{a, b}{ }^{\beta}, \quad Y^{\alpha}{ }_{a b} \rightarrow \kappa^{4} Y^{\alpha}{ }_{, a b}, \quad Y_{\alpha}{ }^{\beta \gamma} \rightarrow \kappa^{4} Y_{\alpha}{ }^{\beta \gamma}, \quad Y_{\alpha, b, c d} \rightarrow-\kappa^{3} Y_{\alpha, b, c d}, \\
& Y_{, b}^{\alpha} \rightarrow-\kappa^{5} Y_{, b}^{\alpha}, \quad Y_{a, b, c d} \rightarrow \kappa^{4} Y_{a, b, c d}, \quad Y_{a,}{ }^{\beta \gamma} \rightarrow-\kappa^{5} Y^{\beta \gamma}, \quad Y_{a b, c d} \rightarrow \kappa^{4} Y_{a b, c d} .
\end{aligned}
$$

For example, we find at dimension 2 ,

$$
Y_{\alpha,}^{\beta}=\frac{1}{4}\left(\gamma^{a b}\right)_{\alpha}^{\beta} Y_{a b}
$$

but at dimension 2.5 ,

$$
Y_{\alpha, b c}=2 \kappa\left(\gamma_{[b}\right)_{\alpha \gamma} Y_{c]}^{\gamma}
$$

\section{C.2 Local Lie superalgebra construction}

Let us now extend the super-Maxwell $\infty$ algebra discussed above to negative dimension elements. To do so, we will employ a construction due to Kac [40] (see also appendix B of [41] which we follow). Its application to our present situation is due to Jakob Palmkvist [42]. We thank Axel Kleinschmidt for parallel comments on the bosonic case.

Kac defined a local Lie superalgebra as a direct sum $\mathcal{T}_{-1} \oplus \mathcal{T}_{0} \oplus \mathcal{T}_{+1}$ of three vector spaces, equipped with a Lie superbracket

$$
\left[\mathcal{T}_{0}, \mathcal{T}_{ \pm 1}\right]=\mathcal{T}_{ \pm 1}, \quad\left[\mathcal{T}_{-1}, \mathcal{T}_{+1}\right]=\mathcal{T}_{0}, \quad\left[\mathcal{T}_{0}, \mathcal{T}_{0}\right]=\mathcal{T}_{0}
$$

where here we use $[\cdot, \cdot]$ as a graded commutator. ${ }^{18}$ The local Lie superalgebra can be extended to a unique superalgebra in two steps:

\footnotetext{
${ }^{18}$ Here we will be a little sloppy and treat vector spaces and their elements interchangeably. We are also interested in the case where odd elements are fermionic and even elements are bosonic, but the construction is general.
} 
1. Freely generate all positive elements at level $k$ by nested $k$ commutators of $\mathcal{T}_{+1}$ with itself, i.e. $\tilde{\mathcal{T}}_{+k}=\left[\mathcal{T}_{+1},\left[\cdots\left[\mathcal{T}_{+1}, \mathcal{T}_{+1}\right]\right]\right]$. Repeat for all negative elements using $\mathcal{T}_{-1}$. The maximal extension of the local Lie superalgebra $\tilde{\mathcal{T}}=\sum_{k} \tilde{\mathcal{T}}_{+k}$ is given by the set of all such freely generated elements.

2. There is a maximal ideal $D$ among the freely generated elements. Quotient out by it, defining $\mathcal{T}=\tilde{\mathcal{T}} / D$. In practice, this amounts to dropping all freely generated positive elements $\tilde{\mathcal{T}}_{+k}$ whose commutator with $\mathcal{T}_{-1}$ vanishes, and similarly for all negative elements $\tilde{\mathcal{T}}_{-k}$ with vanishing $\mathcal{T}_{+1}$ commutator. The resulting algebra $\mathcal{T}$ is the minimal Lie superalgebra generated by the local Lie superalgebra (C.32). The resulting superalgebra is necessarily simple.

This construction applies in our present case, where the positive level generators correspond to what we call the super-Maxwell $\infty$ algebra and the non-positive level generators to $\widehat{\mathcal{H}}_{L}$. The level corresponds to twice the dimension, and the local generators discussed above are

$$
\mathcal{T}_{-1}=M_{\alpha a}, \quad \mathcal{T}_{0}=M_{a b}, \quad \mathcal{T}_{+1}=Q_{\alpha} .
$$

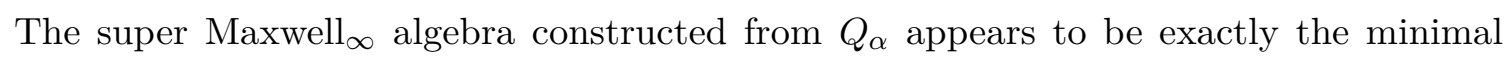
extension of the positive elements $\mathcal{T}_{+k}$. At level 2, we keep only $P_{a}$ in (C.1), this being the only component of $\left\{Q_{\alpha}, Q_{\beta}\right\}$ not annihilated by $M_{\alpha a}$. The other generators uncovered up through level 8 (dimension 4) each have non-vanishing commutator with $M_{\alpha a}$. It is not obvious (but seems likely) that all freely generated elements beyond this point which are consistent with the Jacobi identity must have non-vanishing $M_{\alpha a}$ commutator.

We require one extra element: the existence of a non-degenerate bilinear form $\eta$. This can be constructed recursively, following the general proof given in [41], although in our case $\eta\left(\mathcal{T}_{i}, \mathcal{T}_{j}\right)$ is non-vanishing only for $i+j=2$. It must be non-degenerate because $\mathcal{T}$ is simple. With this in mind, we can define the elements of $\mathcal{T}_{-\Delta}$ as the "duals" of the elements in $\mathcal{T}_{2+\Delta}$. This fixes their normalization and determines their commutation relations with $\left\{Q_{\alpha}, P_{a}, \tilde{Q}^{\alpha}\right\}$ by reflecting the relations from the positive level generators. The Jacobi identity then determines their commutation relations with higher positive elements. Armed with the commutation relations $\left[\mathcal{T}_{-\ell}, \mathcal{T}_{\ell^{\prime}}\right]=\mathcal{T}_{\ell^{\prime}-\ell}$ for $\ell, \ell^{\prime}>0$, one can directly compute $\left[\mathcal{T}_{-\ell}, \mathcal{T}_{-\ell^{\prime \prime}}\right]=\mathcal{T}_{-\left(\ell+\ell^{\prime \prime}\right)}$ for $\ell, \ell^{\prime \prime}>0$ by choosing $\ell^{\prime}=2+\ell+\ell^{\prime \prime}$ and reflecting. This is invaluable because the positive level generators are significantly simpler to characterize.

Open Access. This article is distributed under the terms of the Creative Commons Attribution License (CC-BY 4.0), which permits any use, distribution and reproduction in any medium, provided the original author(s) and source are credited.

\section{References}

[1] W. Siegel, Two vierbein formalism for string inspired axionic gravity, Phys. Rev. D 47 (1993) 5453 [hep-th/9302036] [INSPIRE].

[2] W. Siegel, Superspace duality in low-energy superstrings, Phys. Rev. D 48 (1993) 2826 [hep-th/9305073] [INSPIRE]. 
[3] C. Hull and B. Zwiebach, Double Field Theory, JHEP 09 (2009) 099 [arXiv:0904.4664] [INSPIRE].

[4] C. Hull and B. Zwiebach, The gauge algebra of double field theory and Courant brackets, JHEP 09 (2009) 090 [arXiv: 0908.1792] [INSPIRE].

[5] O. Hohm, C. Hull and B. Zwiebach, Background independent action for double field theory, JHEP 07 (2010) 016 [arXiv: 1003.5027] [INSPIRE].

[6] O. Hohm, C. Hull and B. Zwiebach, Generalized metric formulation of double field theory, JHEP 08 (2010) 008 [arXiv: 1006.4823] [INSPIRE].

[7] O. Hohm and S.K. Kwak, Frame-like Geometry of Double Field Theory, J. Phys. A 44 (2011) 085404 [arXiv: 1011.4101] [INSPIRE].

[8] I. Jeon, K. Lee and J.-H. Park, Differential geometry with a projection: Application to double field theory, JHEP 04 (2011) 014 [arXiv:1011.1324] [INSPIRE].

[9] I. Jeon, K. Lee and J.-H. Park, Stringy differential geometry, beyond Riemann, Phys. Rev. D 84 (2011) 044022 [arXiv: 1105.6294] [INSPIRE].

[10] G. Aldazabal, D. Marqués and C. Núñez, Double Field Theory: A Pedagogical Review, Class. Quant. Grav. 30 (2013) 163001 [arXiv: 1305.1907] [INSPIRE].

[11] D.S. Berman and D.C. Thompson, Duality Symmetric String and M-theory, Phys. Rept. 566 (2014) 1 [arXiv: 1306.2643] [INSPIRE].

[12] O. Hohm and S.K. Kwak, $N=1$ Supersymmetric Double Field Theory, JHEP 03 (2012) 080 [arXiv:1111.7293] [INSPIRE].

[13] I. Jeon, K. Lee and J.-H. Park, Supersymmetric Double Field Theory: Stringy Reformulation of Supergravity, Phys. Rev. D 85 (2012) 081501 [Erratum ibid. 86 (2012) 089903] [arXiv: 1112.0069] [INSPIRE].

[14] A. Coimbra, C. Strickland-Constable and D. Waldram, Supergravity as Generalised Geometry I: Type II Theories, JHEP 11 (2011) 091 [arXiv:1107.1733] [INSPIRE].

[15] I. Jeon, K. Lee, J.-H. Park and Y. Suh, Stringy Unification of Type IIA and IIB Supergravities under $N=2 D=10$ Supersymmetric Double Field Theory, Phys. Lett. B 723 (2013) 245 [arXiv: 1210.5078] [INSPIRE].

[16] O. Hohm, S.K. Kwak and B. Zwiebach, Unification of Type II Strings and T-duality, Phys. Rev. Lett. 107 (2011) 171603 [arXiv:1106.5452] [INSPIRE].

[17] O. Hohm, S.K. Kwak and B. Zwiebach, Double Field Theory of Type II Strings, JHEP 09 (2011) 013 [arXiv:1107.0008] [INSPIRE].

[18] M. Hatsuda, K. Kamimura and W. Siegel, Superspace with manifest T-duality from type-II superstring, JHEP 06 (2014) 039 [arXiv: 1403.3887] [INSPIRE].

[19] M. Hatsuda, K. Kamimura and W. Siegel, Ramond-Ramond gauge fields in superspace with manifest T-duality, JHEP 02 (2015) 134 [arXiv:1411.2206] [INSPIRE].

[20] M. Cederwall, Double supergeometry, JHEP 06 (2016) 155 [arXiv:1603.04684] [INSPIRE].

[21] I. Bandos, Superstring in doubled superspace, Phys. Lett. B 751 (2015) 408 [arXiv: 1507.07779] [INSPIRE].

[22] J.-H. Park, Green-Schwarz superstring on doubled-yet-gauged spacetime, JHEP 11 (2016) 005 [arXiv: 1609.04265] [INSPIRE]. 
[23] H. Godazgar, M. Godazgar, O. Hohm, H. Nicolai and H. Samtleben, Supersymmetric $E_{7(7)}$ Exceptional Field Theory, JHEP 09 (2014) 044 [arXiv: 1406.3235] [INSPIRE].

[24] E. Musaev and H. Samtleben, Fermions and supersymmetry in $E_{6(6)}$ exceptional field theory, JHEP 03 (2015) 027 [arXiv: 1412.7286] [INSPIRE].

[25] A. Baguet and H. Samtleben, E $E_{8(8)}$ Exceptional Field Theory: Geometry, Fermions and Supersymmetry, JHEP 09 (2016) 168 [arXiv: 1607.03119] [INSPIRE].

[26] G. Bossard, A. Kleinschmidt and E. Sezgin, On supersymmetric $E_{11}$ exceptional field theory, JHEP 10 (2019) 165 [arXiv: 1907. 02080] [INSPIRE].

$[27]$ D. Butter, H. Samtleben and E. Sezgin, $E_{7(7)}$ Exceptional Field Theory in Superspace, JHEP 01 (2019) 087 [arXiv:1811.00038] [INSPIRE].

[28] M. Cederwall, Towards a manifestly supersymmetric action for 11-dimensional supergravity, JHEP 01 (2010) 117 [arXiv:0912.1814] [InSPIRE].

[29] M. Cederwall, D=11 supergravity with manifest supersymmetry, Mod. Phys. Lett. A 25 (2010) 3201 [arXiv: 1001.0112] [INSPIRE].

[30] N. Berkovits and M. Guillen, Equations of motion from Cederwall's pure spinor superspace actions, JHEP 08 (2018) 033 [arXiv:1804.06979] [INSPIRE].

[31] M. Poláček and W. Siegel, Natural curvature for manifest T-duality, JHEP 01 (2014) 026 [arXiv: 1308.6350] [INSPIRE].

[32] J. Gomis and A. Kleinschmidt, On free Lie algebras and particles in electro-magnetic fields, JHEP 07 (2017) 085 [arXiv:1705.05854] [INSPIRE].

[33] J. Gomis, A. Kleinschmidt and J. Palmkvist, Symmetries of M-theory and free Lie superalgebras, JHEP 03 (2019) 160 [arXiv:1809.09171] [INSPIRE].

[34] K. Peeters, Introducing Cadabra: A symbolic computer algebra system for field theory problems, hep-th/0701238 [INSPIRE].

[35] K. Peeters, A Field-theory motivated approach to symbolic computer algebra, Comput. Phys. Commun. 176 (2007) 550 [cs/0608005] [inSPIRE].

[36] E. Bergshoeff and M. de Roo, Supersymmetric Chern-Simons Terms in Ten-dimensions, Phys. Lett. B 218 (1989) 210 [inSPIRE].

[37] D.Z. Freedman and A. Van Proeyen, Supergravity, Cambridge University Press, Cambridge, U.K., (2012).

[38] D. Butter, $N=1$ Conformal Superspace in Four Dimensions, Annals Phys. 325 (2010) 1026 [arXiv: 0906 . 4399] [INSPIRE].

[39] E. Lescano, C.A. Núñez and J.A. Rodríguez, Supersymmetry, T-duality and heterotic Q'-corrections, JHEP 07 (2021) 092 [arXiv: 2104.09545] [INSPIRE].

[40] V.G. Kac, Lie Superalgebras, Adv. Math. 26 (1977) 8 [inSPIRE].

[41] G. Bossard, A. Kleinschmidt, J. Palmkvist, C.N. Pope and E. Sezgin, Beyond E11, JHEP 05 (2017) 020 [arXiv: 1703.01305] [INSPIRE].

[42] J. Palmkvist, private communication. 